2003RP-10

\title{
Analyse des événements indésirables liés à la prestation des soins de santé : \\ Démarche structurée et grille d'analyse
}

Nathalie de Marcellis-Warin, Geneviève Dufour

Rapport de projet
Project report

Ce document a été produit dans le cadre du projet « Gouvernance des soins de santé ", financé par le Ministère des Finances du Québec et le Ministère de la Santé et des Services Sociaux du Québec

Montréal

Juin 2003

(C) 2003 Nathalie de Marcellis-Warin, Geneviève Dufour. Tous droits réservés. All rights reserved. Reproduction partielle permise avec citation du document source, incluant la notice (C)

Short sections may be quoted without explicit permission, iffull credit, including (C) notice, is given to the source
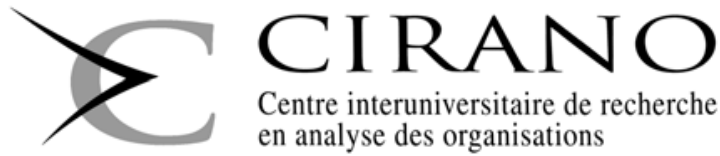

Centre interuniversitaire de recherche en analyse des organisations 


\section{CIRANO}

Le CIRANO est un organisme sans but lucratif constitué en vertu de la Loi des compagnies du Québec. Le financement de son infrastructure et de ses activités de recherche provient des cotisations de ses organisationsmembres, d'une subvention d'infrastructure du ministère de la Recherche, de la Science et de la Technologie, de même que des subventions et mandats obtenus par ses équipes de recherche.

CIRANO is a private non-profit organization incorporated under the Québec Companies Act. Its infrastructure and research activities are funded through fees paid by member organizations, an infrastructure grant from the Ministère de la Recherche, de la Science et de la Technologie, and grants and research mandates obtained by its research teams.

Les organisations-partenaires / The Partner Organizations

PARTENAIRE MAJEUR

. Ministère du développement économique et régional [MDER]

PARTENAIRES

. Alcan inc.

. Axa Canada

. Banque du Canada

. Banque Laurentienne du Canada

. Banque Nationale du Canada

. Banque Royale du Canada

. Bell Canada

. Bombardier

. Bourse de Montréal

. Développement des ressources humaines Canada [DRHC]

. Fédération des caisses Desjardins du Québec

. Gaz Métropolitain

. Hydro-Québec

. Industrie Canada

. Ministère des Finances [MF]

. Pratt \& Whitney Canada Inc.

. Raymond Chabot Grant Thornton

. Ville de Montréal

. École Polytechnique de Montréal

. HEC Montréal

. Université Concordia

. Université de Montréal

Université du Québec à Montréal

. Université Laval

. Université McGill

ASSOCIÉ AU :

. Institut de Finance Mathématique de Montréal (IFM²)

. Laboratoires universitaires Bell Canada

. Réseau de calcul et de modélisation mathématique $\left[\mathrm{RCM}^{2}\right]$

. Réseau de centres d'excellence MITACS (Les mathématiques des technologies de l'information et des systèmes complexes)

ISSN 1499-8610 (Version imprimée) / ISSN 1499-8629 (Version en ligne) 


\title{
Analyse des événements indésirables liés à la prestation des soins de santé : Démarche structurée et grille d'analyse *
}

\author{
Nathalie de Marcellis-Warin ${ }^{\dagger}$, Geneviève Dufour ${ }^{\ddagger}$
}

\begin{abstract}
Résumé / Abstract
Le présent rapport va définir les bases d'un cadre normatif servant à évaluer et à colliger les divers incidents et accidents liés à la prestation des soins dans les centres hospitaliers. Notre objectif est d'une part, d'aider à structurer au sein des établissements l'analyse approfondie des accidents et incidents liés à la prestation des soins tout en gardant une flexibilité pour les hôpitaux qui utilisent déjà d'autres outils et d'autre part, de standardiser l'identification des événements indésirables et l'analyse des causes, des facteurs de contexte et de la gestion des situations à risque pour permettre une remontée de certaines informations au niveau local puis national. Nous allons proposer une démarche structurée pour mener les analyses approfondies et présenter un outil d'analyse (appelé «Grille d'analyse CIRANO », qui s'appuie sur un modèle utilisé dans l'industrie nucléaire validé par de nombreuses études) qui permet d'identifier les vulnérabilités du système de soins dans son ensemble. La Grille d'analyse CIRANO peut servir de base commune aux établissements et permettre une standardisation « minimum » des registres locaux. En effet, il peut être utilisé comme outil d'analyse s'il est intégré dans la démarche proposée ou comme rapport d'analyse pour reporter l'ensemble des résultats de l'analyse dans le cas où d'autres outils d'analyse seraient utilisés. Une étude est en cours pour adapter la démarche et la Grille d'analyse aux autres types d'établissements (CLSC, CHSLD,...).
\end{abstract}

Mots clés : Hôpital, risque, incident/accident, défaillance organisationnelle, grille d'analyse.

\footnotetext{
* Nous tenons à remercier l'ensemble des personnes qui ont contribué à l'élaboration de ce rapport et plus particulièrement Docteure Sylvie Bernier et Huguette Rivard du Ministère de la Santé et des Services Sociaux, les membres du Groupe national d'aide à la gestion des risques et à la qualité, Docteure Micheline Ste-Marie, Claire Arbelot, Michèle Beauchemin Perreault, Docteur Jeffrey Barkun, Michelle Dionne, Docteure Line Duchesne, Docteure Martine Gagnon, Docteure Marie Gourdeau, Nicole Guinard, Docteur Jean Joly, Marilyn Kaplow, Docteure Yolande Leduc, Patricia Lefebvre, Rénald Lemieux, Docteur Bruno Morin, Docteur Claude Rivard et Docteur Denis -Richard Roy pour leurs échanges fructueux tout au long du projet.

Nous tenons aussi à remercier les personnes que nous avons sollicitées à de nombreuses reprises, Isabelle Demers, Martine Dubé, Jocelyne Carl, Anne Lemay, Lorraine Mossman ainsi que les autres personnes que nous avons rencontrées, contactées par téléphone ou par courriel et plus particulièrement Suzanne Allaire, Danielle Bellemare, Alain Boisvert, Sylvie Boulanger, Jocelyne Faille, Myriam Fernandez, Docteur Guillemette, Denis Lebel, Lynda Marcotte, Docteur Nadeau ainsi que Martine Dufresne et l'Association des Hôpitaux du Québec et Élise Amyot, Diane Leblanc et Nathalie Gagné et l'Association Canadienne de Protection Médicale.

Nous souhaiterions remercier par avance les établissements hospitaliers qui ont accepté de participer à la première phase de l'étude pilote de la GRILLE d'analyse CIRANO : le CHUM, l'Hôpital Ste-Justine, l'Hôpital du Lakeshore et le Centre hospitalier régional de Lanaudière. Une deuxième phase suivra avec des établissements hospitaliers et d'autres types d'établissements.

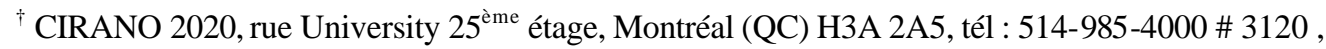
fax : 514-985-4039, courriel : demarcen@ cirano.qc.ca.

¥ CIRANO 2020, Rue University $25^{\text {ème }}$ étage, Montréal (QC) H3A 2A5, tél : 514-985-4000 \# 3120 , fax : 514-985-4039, courriel : genevieve.dufour@cirano.qc.ca.
} 
This report defines a framework to evaluate and collect data on incidents and accidents occurring in hospitals and to create database at local and national level. Our goals are: 1) to provide a structure for thorough analysis within the hospitals that can be used with the already existing tools and 2) to standardise data collected on undesirable events, their causes, contextual factors, and the management of the situation. We propose a step by step method to conduct a thorough investigation and we propose an analysis grid (named "Grille d'analyse CIRANO"), based on a model used in the nuclear industry which has been validated by numerous studies, to identify vulnerabilities of the system. This grid can be used as a commune base in hospitals in order to have a minimum standardisation of local database. We are currently working on adapting this grid to other types of health care facilities (CLSC, CHSLD, ...).

Keywords : Hospital, risk, incident/accident, organisational failure, analysis grid. 
2.1 Le réseau des services de

santé. ..p13

2.2 Evolution de la gestion des risques dans les centres hospitaliers................. p15

2.2.1 Les programmes d'assurance : une impulsion pour la gestion des risque..............p16 2.2.2 La gestion des risques dans les centres hospitaliers : d'une gestion des réclamations à un processus continu d'amélioration de la qualité. .p17

2.2.3. Les différentes initiatives qui coexistent. .p20

2.3 L'implication des ordres professionnels dans la sécurité des patients : l'exemple du nouveau code de déontologie des médecins........................................p25

2.4 Le programme d'agrément et la sécurité des patients.............................p26

2.5 L'importance de la loi 113 et les difficultés rencontrées par les établissements.

3. DEFINITIONS : SYSTEMES COMPLEXES ET DEFAILLANCES............p28

3.1 Le système de soins : un système complexe.......................................p29

3.1.1 Définition d'un système................................................................................. 229

3.1.2 Définition d'un système complexe ..................................................................... 229

3.1.3 Le système de soins et le système « hôpital »....................................................p29

3.2 Les défaillances des systèmes complexes............................................p31

3.2.1 Chaîne d'événements, causes proximales et causes racines..................................p31

3.2.2 Facteur Humain : erreurs humaines et conditions du système favorisant

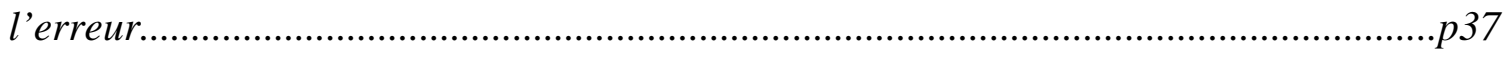




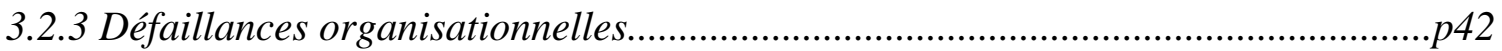

3.2.4 Défaillance technique (équipement/matériel)...................................................p43

4. VERS UN MODÈLE D'ANALYSE APPROFONDIE DE CERTAINS INCIDENTS ET ACCIDENTS LIÉS À LA PRESTATION DES SOINS DE

SANTÉ..................................................................p44

4.1 Présentation du modèle « RECUPERARE » utilisé dans l'industrie nucléaire et de la méthode d'analyse associée à ce modèle.....................................p45

4.1.1 Présentation générale du modèle RECUPERARE .............................................p46

4.1.2 Les spécificités du modèle RECUPERARE......................................................p47

4.1.3. La méthode d'analyse des incidents à partir du modèle RECUPERARE..............p51

4.2. Adaptation du modèle RECUPERARE au système de soins....................p52

4.2.1 Les fondements du modèle RECUPERARE-SANTE ..........................................p53

4.2.2 Le modèle RECUPERARE-SANTE ...................................................................p53

4.3 Grille d'analyse des événements indésirables liés à la prestation des soins élaborée à partir du modèle RECUPERARE-SANTE...............................p65

4.3.1 Présentation de l'outil d'analyse approfondie : la GRILLE d'analyse..................p65

4.3.2 La GRILLE d'analyse CIRANO..................................................................p 67

5. PROPOSITION D'UNE DÉMARCHE STRUCTURÉE D'ANALYSE APPROFONDIE DES INCIDENTS/ACCIDENTS ÉVITABLES LIÉS À LA PRESTATION DES SOINS..................................................p87

5.0 Le déclenchement de l'analyse approfondie....................................p87

5.0.1 Les critères « déclencheurs » généralement retenus...........................................p88

5.0 .2 Les critères qui pourraient être retenus.............................................................p 91

5.1 Les étapes de la démarche d'analyse approfondie................................p95

CONCLUSION.....................................................................p101 


\section{INTRODUCTION}

"Mistakes are a fact of life. It's the response to errors that counts"

Nikki Giovanni (American poet)

Erreur de médicament, surdose de médicament, administration d'un médicament par la mauvaise voie, infection brs d'une transfusion, utilisation d'un outil non stérilisé, chutes, utilisation d'un gaz anesthésiant à la place d'oxygène, intervention sur le mauvais membre, défibrillateur inopérant, interversion des échantillons au laboratoire, résultats d'un test mis dans le mauvais dossier aux archives, blessure lors du transfert d'un patient sur la table de radiologie, brûlure,...

Au cours de ces dernières années, dans plusieurs pays, la sécurité des patients et la survenance d'événements indésirables liés à la prestation des soins de santé sont devenus d'importants sujets de préoccupation tant sur le plan des politiques que sur le plan des pratiques en soins de santé. Un rapport de l'Institute of Medicine publié en novembre 1999, To Err Is Human, a révélé que les accidents survenus lors de la prestation des soins pouvaient être à l'origine de 44 000 à 98000 décès par année dans les hôpitaux de soins de courte durée aux États-Unis, ce qui en ferait la $5^{\text {me }}$ cause de décès dans ce pays. De plus, ces accidents augmenteraient la durée d'hospitalisation (en moyenne de 1 à 4 jours par patient ayant subi un préjudice) et les coûts de traitement (coût des examens supplémentaires,...). Une étude a montré que le coût total associé représenterait environ $2 \%$ des dépenses totales de santé aux États-Unis. Cet axe de recherche connait un intérêt grandissant qui s'explique par la mortalité, la prolongation des séjours hospitaliers et l'augmentation des coûts inhérents quils engendrent. En Australie, une étude révèle que le ratio accidents par hospitalisation est significatif et que $50 \%$ des accidents sont évitables. 
Même si actuellement aucune information n'est collectée sur les accidents survenus lors de la prestation de soins au Québec, le rapport du comité ministériel Francoeur publié en 2001 conclut qu'il n'y a aucune raison que le ratio ne soit pas du même ordre, ce qui a suscité de nombreux questionnements concernant notamment leur incidence, leurs causes et les mesures de prévention pouvant être mises en place (MSSS, 2001). Un projet de «recherche-action » sur les accidents évitables dans la prestation des soins a été lancé par le Ministère de la Santé et des Services Sociaux (MSSS) après la publication de ce rapport. Le mandat a été confié à la direction générale des affaires médicales et universitaires du MSSS qui a été chargée d'évaluer et d'appliquer les recommandations du comité en collaboration avec les régies régionales, les établissements de santé, les ordres professionnels, les assureurs, les universités, le coroner et les autres intervenants. Le Groupe national d'aide à la gestion des risques et à la qualité (GNAGRQ) a été mis sur pied en septembre 2001. L'une de ses fonctions est d'élaborer des outils de recherche, de gestion et d'analyse des données afin d'avoir une meilleure connaissance du profil des accidents évitables, des facteurs et des circonstances qui contribuent à leur occurrence et ainsi concevoir les mesures propres à en prévenir la répétition.

La nouvelle Loi 113 sur la prestation des soins sécuritaires dans les établissements du réseau de la santé et des services sociaux du Québec adoptée en décembre 2002 (cf annexe 1) fait suite à ce projet. La loi oblige les professionnels à déclarer tous les incidents et accidents survenus lors de la prestation des soins et à divulguer les accidents aux patients. Dans son article 8, elle spécifie que «tout usager a le droit d'être informé, le plus tôt possible, de tout accident survenu au cours de la prestation de services qu'il a reçue et susceptible d'entraîner ou ayant entraîné des conséquences sur son état de santé ou son bien-être ainsi que des mesures prises pour contrer, le cas échéant, de telles conséquences ou pour prévenir la récurrence d'un tel accident ». Cette loi prévoit également l'obligation pour tout établissement de mettre en place un comité de gestion des risques et de la qualité, lequel aura pour fonctions de rechercher, de développer et de promouvoir des moyens visant à assurer la sécurité des usagers et à réduire l'incidence des effets indésirables et des accidents liés à la prestation des services de santé et 
des services sociaux. La loi spécifie la mise en place d'un registre local et national des incidents et accidents.

Le projet de recherche CIRANO sur la gestion des risques dans les hôpitaux s'inscrit dans le cadre de la loi 113. Nous avons été mandatés par le MSSS et le Groupe national d'aide à la gestion des risques et à la qualité présidé à l'époque par le Dr Denis-Richard Roy (remplacé par le Dr Micheline Ste Marie) pour définir les bases d'un cadre normatif servant à évaluer et à colliger les divers incidents et accidents liés à la prestation des soins dans les centres hospitaliers et développer un outil d'analyse des défaillances systémiques. En effet, la plupart des événements indésirables liés à la prestation des soins proviennent d'une défaillance du système et non d'une personne clairement identifiée. Les établissements se limitent souvent à rechercher les responsabilités individuelles. La prise en compte du facteur humain et des interactions complexes entre les individus, les produits, la technologie et les systèmes peut permettre d'améliorer la sécurité des patients dans les hôpitaux.

Nous avons concentré notre étude sur les centres hospitaliers $(\mathrm{CH})$. Nous avons recueilli des informations sur les autres types d'établissements du réseau mais une autre étude est actuellement en cours. Nous avons étudié l'ensemble du processus de report des accidents et incidents et les formulaires de déclaration utilisés, le AH-223 ou un autre formulaire. L'analyse critique du formulaire AH-223 a fait l'objet d'un autre rapport ${ }^{2}$. Les définitions données par la loi renseignent du type d'événement à reporter :

Accident : Action ou situation où le risque se réalise et est, ou pourrait être, à l'origine de conséquences sur l'état de santé ou le bien-être de l'usager, du personnel, d'un professionnel concerné ou d'un tiers.

\footnotetext{
${ }^{2}$ De Marcellis-Warin N., G. Dufour, 2003, «Déclaration des incidents et des accidents dans les centres hospitaliers - Analyse critique du rapport d'incident/accident AH-223 et de la structure de gestion de ces rapports », rapport de recherche CIRANO remis au MSSS, avril.
} 
Incident : Une action ou une situation qui n'entraîne pas de conséquence sur l'état de santé ou le bien-être d'un usager, de personnel, d'un professionnel concerné ou d'un tiers mais dont le résultat est inhabituel et qui, en d'autres occasions, pourrait entraîner des conséquences.

L'objectif du projet de recherche CIRANO est d'une part, d'aider à structurer l'analyse interne des risques d'accidents et d'incidents en milieu hospitalier tout en gardant une flexibilité pour les établissements qui utilisent déjà d'autres outils et d'autre part, de permettre une standardisation du report des données et de l'analyse des causes et des facteurs de contexte pour permettre une remontée de certaines informations au niveau local et national .

Nous allons dans la section 2 étudier la situation actuelle au Québec en matière de gestion des risques et de la qualité dans les établissements hospitaliers. Dans la section 3, nous allons définir la notion de système complexe et identifier les différents types de défaillances. La section 4 présentera un modèle d'analyse des incidents et des accidents lié à la prestation des soins adapté d'un modèle de retour d'expérience (RECUPERARE) utilisé dans l'industrie nucléaire. Le modèle examine l'émergence des causes de l'incident/accident, les caractéristiques du déroulement de l'incident/accident et la récupération qui peut être ex ante dans le cas où cela ne touche pas le patient - les «échappées belles » - et ex post si cela touche le patient - les «gestes posés» pour stabiliser la situation et diminuer les conséquences potentielles. La méthode d'analyse associée à ce modèle (la GRILLE d'analyse CIRANO) permet d'identifier les vulnérabilités du système de soins dans son ensemble et de mettre en évidence les causes humaines, techniques et organisationnelles et les facteurs de contexte. Cette méthode permet aussi d'apprendre de la gestion des incidents et des accidents passés. En effet, l'originalité du modèle est de mettre en évidence les délais de latence, de détection et de récupération, qui sont regroupés sous le terme performance. Cette analyse approfondie doit permettre à l'établissement de tirer les leçons des «expériences » passées. L'utilisation de la grille d'analyse approfondie va s'imbriquer dans une démarche structurée que nous présenterons en section 5. 


\title{
CHEMINEMENT DU PROJET DE RECHERCHE
}

\author{
Étape 1 - Démarrage du projet \\ Revue de littérature sur les défaillances des systèmes complexes \\ et les outils d'analyse existants dans d'autres industries
}

L'étape de démarrage a consisté en l'élaboration du plan de travail du projet et une revue de littérature sur l'intérêt d'utiliser des outils et les démarches d'analyse utilisés dans d'autres industries. Nous avons dressé une première liste de personnes à contacter. Le CNAGRQ et le MSSS nous ont fourni une liste de professionnels de la santé.

\section{Étape 2 - Analyse de la situation actuelle au Québec}

Nous avons tout d'abord étudié les procédures de collecte des donnés d'incidents et d'accidents en place (rapport d'incident/accident utilisé, collecte, suivi des rapports, structure de gestion) et les outils d'analyse existants. Nous avons consulté les organismes concernés (l'AHQ, l'ACPM,...) et les établissements qui ont déjà une structure en place ou un projet en cours. L'objectif pour la première étape était de recueillir de l'information pertinente sur la situation actuelle au Québec.

\section{Etape 3 - Proposition d'une GRILLE d'analyse approfondie des événements indésirables liés a la prestation des soins et consultations des experts et intervenants du réseau concernés}

Nous avons élaboré une grille d'analyse approfondie adaptée d'un modèle d'analyse appelé RECUPERARE utilisé dans l'industrie nucléaire. Cette grille pourrait être utilisée par les établissements pour l'analyse approfondie de certains incidents et accidents.

\section{=> GRILLE D'ANALYSE CIRANO VERSION 0}

Au cours de la deuxième étape des consultations, nous avons pris contact avec des experts choisis et des intervenants de divers établissements afin de solliciter leurs opinions et des suggestions sur la GRILLE d'analyse proposée. Ces experts et intervenants ont été repérés au cours de la première étape. Le but de cette seconde série de consultations était de :

. solliciter des commentaires sur le rapport AH-223 et sur la GRILLE CIRANO (objectifs, portée, fonctions, données recueillies);

. solliciter des commentaires sur les sections et sous-sections proposées ;

. déterminer quels sont les facteurs de succès et les difficultés et préoccupations reliées à l'utilisation d'un tel outil d'analyse ; 


\section{Étape 4 - Analyse des remarques et des propositions}

Cette étape a consisté en une analyse de l'information recueillie au cours des différentes rencontres pour améliorer la grille d'analyse. Certaines sections et sous-sections de la grille proposée ont été améliorées en se fondant sur les informations recueillies.

\section{=> GRILLE D'ANALYSE CIRANO VERSION 1}

\section{Étape 5 - Proposition d'une démarche structurée d'analyse approfondie}

L'utilisation de la grille d'analyse approfondie va s'imbriquer dans une démarche structurée d'analyse approfondie (notamment pour savoir dans quel cas il est recommandé ou nécessaire de faire ce type d'analyse et le suivi). Nous avons détaillé chacune des étapes. Les établissements pourront utiliser la GRILLE CIRANO comme outil d'analyse s'il est intégré dans la démarche proposée ou comme rapport d'analyse pour reporter l'ensemble des résultats de l'analyse dans le cas où d'autres outils d'analyse seraient utilisés. En effet, le rapport d'analyse (qui sera en quelque sorte le résumé de l’analyse) devra être standardisé et compilé dans la base de données locale de l'établissement.

\section{Étape 6-Etude pilote \\ de la grille d'analyse CIRANO et de la démarche et d'un plan de développement}

L'objet de l'étude pilote qui va débuter fin mai 2003 est de mettre en place la démarche structurée proposée et d'utiliser la "grille d'analyse des événements indésirables liés au système de soins" élaborée par le CIRANO comme outil d'analyse pour les incidents/accidents. La première phase de l'étude pilote, qui va durer six mois va avoir plusieurs phases et comprendre un échantillon représentatif des établissements de soins de la province (la premier phase comprends 3 hôpitaux à Montréal et 1 hôpital en région). Cette étude devrait permettre de valider la pertinence de l'outil développé et de savoir s'il remplit bien les objectifs souhaités (structurer l'analyse des accidents, identifier les causes et les facteurs de contexte, mettre en évidence les aspects de défaillances organisationnelles, évaluer la gestion de l'incident, identifier les mesures de prévention à mettre en place). Nous souhaiterions évaluer s'il est adapté à la réalité des cas analysés (pertinence des sections, points positifs et négatifs, problèmes rencontrés,...) et le temps mis pour remplir la grille d'analyse. La deuxième phase de l'étude pilote se fera auprès d'autres types d'établissements.

\section{=> VERS UNE GRILLE D'ANALYSE CIRANO VERSION 2...}




\section{LA SITUATION ACTUELLE AU QUÉBEC}

Notre analyse de la situation actuelle de la gestion des risques au Québec est basée sur des visites d'établissements hospitaliers et sur nos échanges et contacts téléphoniques avec des professionnels du milieu de la santé. Nous avons étudié les procédures de collecte des donnés d'incidents et d'accidents en place (rapport d'incident/accident utilisé, collecte, suivi des rapports, structure de gestion), les outils d'analyse existants et la remontée d'informations. Nous avons consulté certains organismes (l'AHQ, l'ACPM,...) et les établissements qui ont déjà une structure d'analyse en place ou un projet en cours pour bien s'informer de leurs orientations. L'étude sur les procédures de collecte et le formulaire utilisé par les établissements a fait l'objet d'un rapport séparé. Nous rappellerons certains points pertinents mais nous allons surtout nous concentrer sur la démarche d'analyse et les outils utilisés.

\subsection{Le réseau des services de santé ${ }^{3}$}

Le système de santé et de services sociaux constitue un réseau qui a pour but le maintien et l'amélioration de la capacité physique, psychique et sociale des personnes. Les établissements de santé et de services sociaux sont administrés sur une base régionale et plusieurs assument plus d'une mission. On compte 126 centres hospitaliers $(\mathrm{CH}), 337$ centres d'hébergement et de soins de longue durée (CHSLD), 147 centres locaux de services communautaires (CLSC) offrant des services de première ligne, 91 centres de réadaptation et 19 centres de protection de l'enfance et de la jeunesse. Les établissements délivrant des soins de court séjour sont les centres hospitaliers de soins généraux et spécialisés et les centres hospitaliers de soins psychiatriques. Les centres hospitaliers universitaires (CHU) sont présents dans trois grandes villes (Montréal, Québec, Sherbrooke). Ces établissements gèrent en général plusieurs sites. Les régies régionales de la santé et des services sociaux doivent, agir à titre de maître-d'oeuvre 
dans la planification, la mise en oeuvre, l'organisation et la coordination des programmes et des services, ainsi que dans l'allocation des ressources sur le territoire. On retrouve également plus de 1000 cliniques médicales privées et, en 1999-2000, le Ministère a subventionné 2700 organismes communautaires. Le graphique suivant représente l'organisation du réseau de la santé :

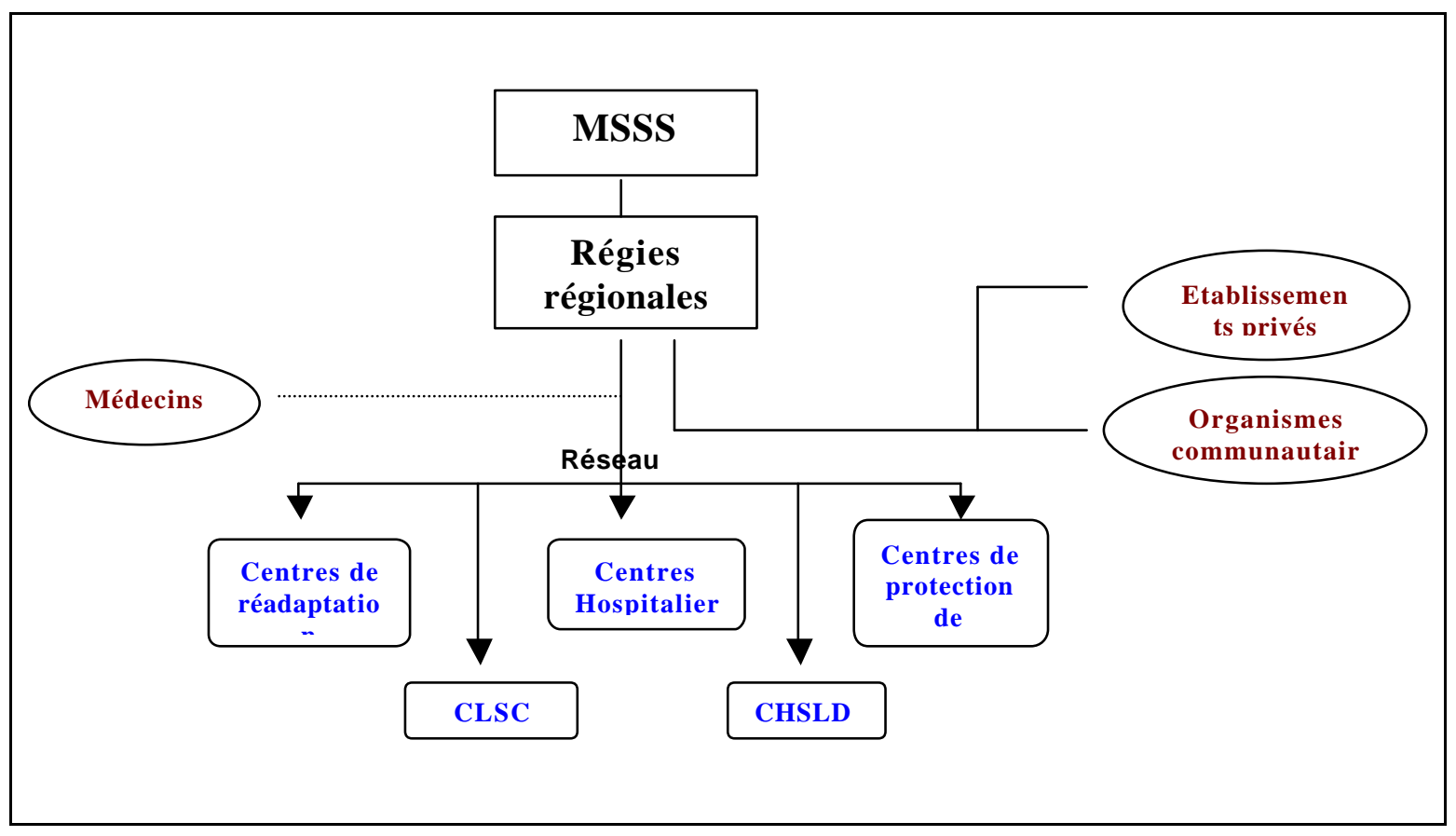

Graphique 1 : L'organisation du réseau des services de santé (adapté de l'organigramme proposé par le MSSS)

La sécurité de l'usager et la qualité des soins sont des priorités pour le réseau. Il est donc important de représenter l'USAGER (ou le bénéficiaire ou le patient ou le client) qui est au cœur du réseau et des préoccupations de chaque établissement de ce réseau. Prenons l'exemple des centres hospitaliers $(\mathrm{CH})$. Le graphique d-dessous représente un hôpital X (et les différents services qui le composent), son environnement proche (avec les autres établissements du réseau) et son environnement plus large (environnement législatif et budgétaire).

\footnotetext{
${ }^{3}$ Source : site du MSSS http://www.msss.gouv.qc.ca
} 


\section{Environnement:législatif, budgétaire}

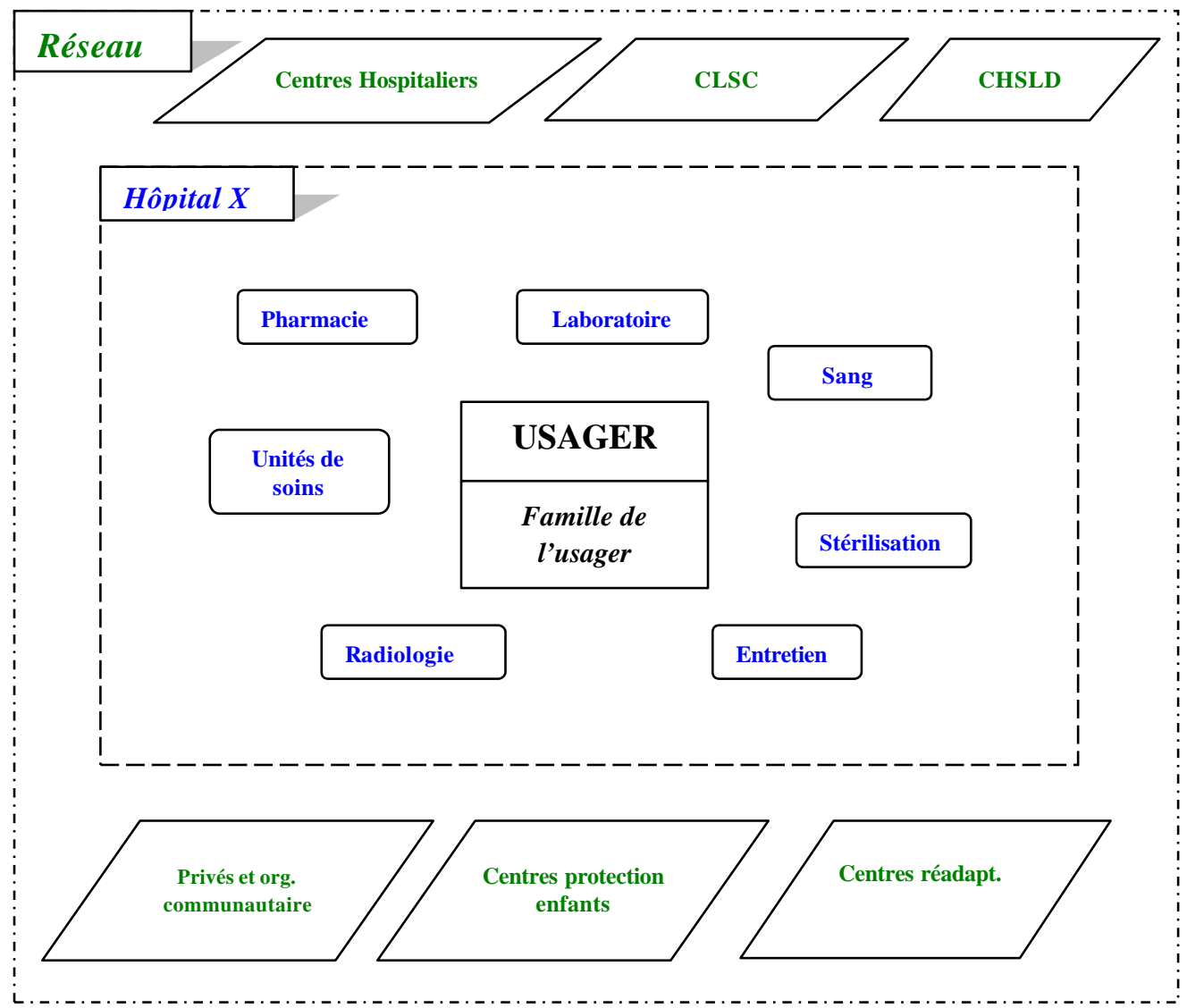

Graphique 2 : Représentation du réseau de la santé du point de vue du CH avec l'usager au centre

\subsection{Evolution de la gestion des risques dans les centres hospitaliers}

La gestion des risques a longtemps été considérée comme une gestion des réclamations. Ce sont les programmes d'assurance qui ont donné une impulsion importante à la mise en place des processus de gestion des risques dans les établissements, bien que fort variables d'un établissement à un autre. En effet, même si des programmes de gestion des risques sont en place dans certains établissements (surtout les CHU et les grands hôpitaux), de nombreux établissements n'ont pas de véritable programme ni de personne en charge exclusivement de ce 
dossier. En effet, même si on a observé une recrudescence des postes de gestionnaires de risques dans les hôpitaux québécois au début des années 90, ce sont les premiers postes qui ont été touchés par les restrictions budgétaires en 93-94, et qui n'ont majoritairement pas été repourvus depuis. Ainsi, dans la plupart des cas la gestion des dossiers de réclamation est faite au niveau de la direction financière et a donc un caractère exclusivement financier. Toutefois, la gestion des risques et de la qualité commence à prendre une place importante dans la gestion hospitalière et est tournée de plus en plus vers une démarche de prévention.

\subsubsection{Les programmes d'assurance : une impulsion pour la gestion des risques}

Tous les établissements hospitaliers s'assurent en responsabilité civile et professionnelle auprès du Regroupement des programmes d'assurances du réseau de la santé et des services sociaux. Cet organisme a été créé en 1986 sur l'initiative de l'Association des Hôpitaux du Québec (AHQ) qui comprend tous les hôpitaux du Québec. Il s'est élargi en 1995 avec l'adhésion d'autres associations d'établissements (CLSC, CHSLD, centres de réadaptation, centre jeunesse et régies régionales). Le regroupement joue aussi le rôle de conseiller auprès de ses membres. Il aide les établissements hospitaliers à mettre en place un programme de gestion des risque et propose des séances de formation et d'information. Toutefois, les établissements ne signalent pas à leur assureur tous les événements donnant lieu à un rapport d'incident /accident. En fait, ne sont signalées que les situations pouvant donner ouverture à une réclamation. Dans certains cas même, des réclamations arrivent à l'assureur alors qu'aucun dossier n'a été déposé par l'établissement.

L'Association Canadienne de Protection Médicale (ACPM) est le principal assureur des médecins. Elle possède un ensemble d'informations sur les causes des poursuites judiciaires entamées qui sont diffusées aux médecins pour réduire leur exposition aux risques. La gestion des risques en milieu hospitalier n'est pas une préoccupation nouvelle pour cette association. 
Déjà en 1988, l'association lançait un appel à l'engagement des médecins ${ }^{4}$ dans les programmes de gestion des risques et de la qualité des centres hospitaliers.

\subsubsection{La gestion des risques dans les centres hospitaliers : d'une gestion des réclamations à un processus continu d'amélioration de la qualité}

Comme on l'a vu précédemment, la gestion des risques a longtemps ressemblé à une gestion des plaintes et des réclamations. Avec la nouvelle loi, «la gestion des risques est passée d'une préoccupation strictement financière à un objectif de sécurité du patient. Elle contribue à l'amélioration continue de la qualité des soins et services offerts à la clientèle et à rendre ces soins de services le plus sécuritaire possible $»^{5}$. La gestion des risques commence par l'identification des situations à risque et par la déclaration des incidents et des accidents. Des systèmes d'information sur les usagers en matière d'hospitalisation existent (par exemple, MedEcho pour les centres hospitaliers de soins de courte durée) mais ils ne permettent pas d'obtenir des informations complètes sur les accidents (même si au départ Med-Echo comportait une section «accidents en cours de soins », les informations étaient souvent inutilisables et trop délicates à gérer).

\section{- La déclaration des incidents/accidents}

Le rapport de déclaration d'incident/accident le plus utilisé est le formulaire AH-223 que distribue le ministère de la santé et des services sociaux aux établissements de santé du réseau. Ce rapport facilite et normalise la cueillette d'informations susceptibles de permettre l'identification des risques. Toutefois, plusieurs établissements ont cherché aux cours des dernières années à adapter le rapport d'incident/accident à leurs besoins spécifiques et on trouve dans certains établissements des rapports «maison». Certains services en particulier

\footnotetext{
${ }^{4}$ Voir ACPM bulletin d'information, automne 1988 vol. 3

${ }^{5}$ Extrait de la présentation de Michelle Dionne de l'AHQ lors du Colloque sur la sécurité des patients qui s'est déroulé à Montréal début mai.
} 
utilisent des rapports qu'ils ont conçus (c'est essentiellement le cas de la pharmacie) et font une gestion des incidents et accidents au niveau du service. Dans d'autres services, il n'y a pas de politique de report (on a observé cela pour le service des archives ou la radiologie), ni de structure définie de suivi et de remontée de l'information. Cette situation peut s'expliquer par la culture du milieu, l'absence de consignes claires dans les services autres que les soins et par le fait que le formulaire $\mathrm{AH}-223$ est construit pour les événements en lien direct avec l'usager.

\section{- La gestion des rapports d'incidents/accidents}

Ces rapports sont le plus souvent remplis par des infirmières et les accidents les plus fréquents sont les chutes et les erreurs de médication. Toutefois, les procédures de suivi de ces rapports ne sont pas toujours clairement définies dans les établissements. Dans la plupart des cas, la gestion des rapports d'incidents/accidents se fait au niveau de la direction des soins infirmiers. Les politiques et les procédures de gestion des rapports sont établies par chaque établissement. Ces politiques et procédures, en plus de varier grandement d'un établissement à un autre, ne sont pas nécessairement clairement définies et diffusées parmi le personnel. Suite à l'adoption de la loi 113, une démarche de réflexion et de sensibilisation à la déclaration des incidents/accidents semble s'être amorcée dans de nombreux établissements.

\section{- La codification et l'analyse des données}

Une fois l'information recueillie, sans regard à sa qualité, elle doit être transmise, codifiée et analysée, sinon, il n’y a aucune utilité à la recueillir. Certains établissements ont une structure bien définie d'analyse statistique et un suivi efficace. Plusieurs établissements ont une base de données sur les incidents/accidents. Pour la saisie et le traitement statistique, certains utilisent un logiciel acheté (CH-CA ou Gesrisk) ou ont développé leur propre logiciel. Dans la plupart des cas, il s'agit de statistiques descriptives pour les sections à choix multiples (c'est à dire qu'il n'y a aucune codification des questions ouvertes). Ces données sont saisies par une tierce personne non impliquée dans les événements et en général plusieurs mois après. De plus, cela prend du 
temps car si il y a codage des questions ouvertes car la personne doit déchiffrer ce qui est écrit ou «compléter» le rapport. Elle essaye de cocher les cases à partir de la section F, Description des faits.

D'autres établissements fonctionnent en identifiant d'abord la catégorie de l'incident ou de l'accident, les mêmes que celles du AH-223. Ensuite des objets et des sous-objets ont été précisés détaillant plus les possibilités du AH-223 (par exemple, ajournement/délai). Les facteurs contributifs sont identifiés si possible, à partir essentiellement de la description des faits. Dans les exemples que l'on a vus, la liste est assez limitée. Certains établissements essayent aussi de codifier les avis et recommandations selon les différentes possibilités : discussion avec la famille, l'usager, le département, l'employé, l'infirmière, le médecin, la pharmacien,..., rapporté à la DSP ou à l'AHQ, révision de la procédure, etc.

Certains établissements cherchent à croiser les informations pour mieux les interpréter. Par exemple, CHUTE-TROUVÉ PAR TERRE est l'objet le plus souvent coché. Il est possible d'établir que le grand nombre de chutes provient d'un département spécifique comme la neuropsychologie et qu'en incluant la variable de la condition préalable on peut montrer que les chutes surviennent surtout lors de crise d'épilepsie. De plus, il peut y avoir des croisements entre l'objet et les conséquences ou l'objet, le service et les conséquences. Ce type d'information permet par la suite au gestionnaire d'avoir une idée des secteurs ou des pathologies particulièrement à risques et permet de fixer des objectifs sur la réduction des risques. Il s'agit d'un début d'analyse qui va au-delà du traitement statistique simple.

\section{- L'analyse approfondie de certains accidents et incidents}

Le rapport d'incident/accident est fait pour déclarer une situation à risque, que le risque se soit ou non réalisé. Dans certains cas, il est important de faire une analyse plus approfondie de la situation pour comprendre ce qui s'est passé. Il est évident qu'il ne faut pas faire d'analyse approfondie pour chaque incident ou accident déclaré. La plupart du temps, ces enquêtes sont 
faites pour des accidents graves. Même si certains établissements nous ont parlé des méthodes de «root causes analyses », il semble que ces méthodes soient utilisées de façon très ponctuelle et pour des accidents exceptionnels (la méthode est assez lourde car elle demande la formation d'une équipe pour faire l'analyse). De plus, des outils développés pour la gestion de la qualité sont parfois appliqués à des situations accidentelles. Par exemple, le diagramme d'Ishikawa (ou diagramme en arrêtes de poisson) est parfois utilisé. Il permet de représenter de manière hiérarchisée les différentes causes ou facteurs pouvant avoir un impact sur le déroulement d'un processus ou être générateur de dysfonctionnements.

\section{- Le processus continu d'amélioration de la qualité}

On a observé que des programmes «qualité » sont mis en place dans la plupart des établissements et que la gestion des risques est prise en compte dans ces programmes. Des actions commencent à être menées mais jusqu'à présent cela se faisait de façon dispersée et sans coordination effective. Avec la loi 113, la gestion des risques s'imbrique clairement dans le processus d'amélioration de la qualité de l'établissement.

\subsubsection{Les différentes initiatives qui coexistent}

Dans les établissements de santé, de nombreuses initiatives, qu'elles soient d'origine professionnelle, managériale ou réglementaire, visant à maîtriser les différents risques identifiés coexistent. Elles sont le plus souvent conçues de manière thématique : risques médicamenteux, infections nosocomiales, risque transfusionnel, sécurité,... Cependant, cette approche des différents risques hospitaliers a conduit à une gestion des risques éclatée (ANAES, 2003). Or, l'absence d'une vision globale des risques rend leur maîtrise difficile. De plus, même si certains risques sont assez bien pris en compte par certains établissements (ex, risques médicamenteux, infections nosocomiales,...), d'autres le sont insuffisamment malgré leur importance (iatrogénie des actes médicaux et des soins). Il sera important d'imbriquer et de coordonner l'ensemble de 
ces initiatives dans le processus global de gestion des risques et de la qualité des établissements. Nous allons présenter deux exemples d'initiatives que nous avons observées dans certains établissements : l'adoption d'un processus d'utilisation des médicament plus sécuritaire et la prévention des infections nosocomiales ${ }^{6}$.

\section{- Vers un processus d'utilisation des médicaments plus sécuritaire}

Un nombre considérable d'accidents évitables est relié à la médication ${ }^{7}$. Une littérature abondante est disponible sur le sujet. Le rapport de l'IOM (IOM, 1999) incite les établissements à adopter un système de distribution sécuritaire et efficient; à revoir les processus reliés à la gestion des médicaments (de la sélection des molécules à l'administration et à la surveillance des résultats); à évaluer et mettre en place les technologies de l'information; à évaluer et implanter l'automatisation des processus et à divulguer l'information sur les médicaments aux patients et aux professionnels de la santé.

Les services de pharmacie des établissements du réseau ont pour la plupart largement témoigné leur intérêt en promouvant auprès des directions l'adoption d'un processus d'utilisation des médicaments plus sécuritaire. Les pharmaciens en établissement sont membres à part entière de l'équipe médicale. Afin d'aider les départements de pharmacie des établissements de santé au Québec, le conseil d'administration de l'Association des pharmaciens des établissements de santé du Québec (l'A.P.E.S) ${ }^{8}$ a constitué un groupe de travail sur les accidents pharmaceutiques pour soutenir les initiatives locales favorisant la prestation sécuritaire de services de santé qui recourent à l'utilisation des médicaments ${ }^{9}$. De façon plus précise, son mandat est de :

\footnotetext{
6 Nous avons volontairement choisi les infections nosocomiales car même si ce type d'accidents évitables a un traitement à part dans les établissements, il faudra penser à les inclure dans le registre local (tout comme les risques transfusionnels) et national. En effet, il ne faut pas multiplier les bases de données.

${ }^{7}$ Cf Lefebvre et Beauchemin Perreault (2001)

${ }^{8}$ L'A.P.E.S. est une association qui regroupe tous les pharmaciens des établissements de santé au Québec soit 1000 professionnels œuvrant dans plus de 250 établissements.

${ }^{9}$ cf mémoire de l'APES remis pour la loi 113
} 
Proposer une définition d'accident pharmaceutique.

Répertorier les systèmes de classification et recommander un système de documentation (incidence, sévérité-conséquences).

> Répertorier les outils d'analyse de système pour évaluer les erreurs potentielles reliées au système de prestation des soins et services.

Élaborer les besoins en formation à l'égard des accidents pharmaceutiques, tels que le rôle de l'Institute for Safe Medication Practice (division canadienne) et l'analyse de la cause fondamentale (Root cause analysis).

Ces initiatives doivent se faire en collaboration avec l'ensemble des professionnels de la santé. Mais la vision d'analyse systémique proposée par le groupe de travail va dans ce sens. En effet, il est important de prendre en compte tous les intervenants dans la chaîne de distribution du médicament et identifier le(s) maillon(s) le(s) plus faible(s). Un patient qui est transféré d'un service à un autre et qui est vu par des médecins différents ne doit pas recevoir deux types de médication qui pourraient interagir. Ainsi, même si le leadership vient de la pharmacie, il faut sensibiliser l'ensemble des intervenants dans l'établissement.

\section{- La prévention des infections nosocomiales}

Les infections nosocomiales figurent au deuxième rang des accidents évitables après les erreurs médicamenteuses (Gourdeau 2003). Les infections nosocomiales font actuellement l'objet d'un traitement à part dans les établissements. Sont reportées sur le AH-223 uniquement les infections qui font ou qui pourraient faire suite à un événement indésirable : par exemple, une infection suite à la réutilisation d'un outil à usage unique ou d'un appareil non stérilisé,... Il s'agit d'une petite part des infections contractées par les usagers du réseau. 
Un programme structuré ou du moins des activités de surveillance épidémiologique ont été mises en place dans un grand nombre d'établissements ${ }^{10}$. L'objectif est de détecter les infections nosocomiales et les facteurs qui y sont associés. Différents points vont caractériser ce programme : le choix du type de surveillance (globale, périodique de prévalence, sélective de l'incidence); la définition des infections surveillées; la méthode de collecte de données; la méthode de compilation et d'analyse des données et la méthode de diffusion des rapports. La surveillance des infections nosocomiales met parfois en évidence des situations qui nécessitent une investigation, soit parce que l'on observe: une augmentation inhabituelle significative de l'incidence d'une infection; des taux dépassant un seuil jugé acceptable selon les normes reconnues et la présence d'infections jugées prioritaires parce qu'elles sont graves, même si le nombre de cas est restreint. Ces investigations sont très techniques et demandent une expertise dans le domaine. En effet, il est souvent difficile de déterminer si l'infection a réellement été contractée pendant l'hospitalisation (et donc liée à la prestation des soins) ou si le patient était déjà atteint à son admission. Le moment de sa détection ne permet pas toujours d'avoir cette information.

De plus, les infections nosocomiales sont généralement la résultante de plusieurs facteurs et il est difficile de les prévenir par une intervention unique (Gourdeau, 2003). Le développement et la mise à jour des politiques et procédures doivent s'effectuer en collaboration avec les différentes unités de soins, départements et services de l'établissement. Différents programmes, allant dans ce sens, sont déjà en place. Ils sont orientés vers la prévention et la qualité des soins. Nous allons en présenter trois :

\footnotetext{
${ }^{10}$ Pour certains établissements, il s'agit d'un programme récent à cause du manque de ressource humaine dans le domaine.
} 
- Le programme de prévention des infections urinaires: Indications d'une cathétérisation; Insertion et maintien d'une sonde; Prélèvements aseptiques; Entretien du matériel d'appoint; ...

- Le programme de prévention des plaies opératoires : Préparation pré-opératoire du patient; Préparation de l'équipe chirurgicale; tenue vestimentaire; brossage chirurgical; Préparation et entretien du bloc opératoire; instruments;

- Le programme sur l'utilisation des solutions antiseptiques et désinfectantes Principes généraux; Sélection des produits; Choix et indications; Entretien du matériel dans divers services de l'hôpital; endoscopes - centre d'exploration fonctionnelle; cathéters, ...

La formation du personnel comprend un volet orientation pour le nouveau personnel et un volet formation en cours d'emploi qui vise à promouvoir, renforcer et soutenir les programmes de prévention présentés ci-dessus. Toutefois, la surveillance des infections doit se fire par du personnel spécialisé en prévention des infections. Le manque de personnel qualifié semble être un obstacle pour certains établissements.

La constitution d'une base de donnée locale et nationale est primordiale pour ce type d'accident évitable. Même si les démarches d'analyse sont différentes des autres types d'accidents, il sera important de standardiser la collecte des données et de les inclure dans la base de donnée nationale. Le Comité des infections nosocomiales du Québec travaille sur ces aspects. Nous envisageons de les rencontrer pour la suite de nos travaux de recherche. 


\subsection{L'implication des ordres professionnels dans la sécurité des patients : l'exemple du NOUVEAU CODE DE DEONTOLOGIE des médecins}

Les ordres professionnels prennent de plus en plus en compte les problèmes liés à la sécurité des patients dans l'exercice de soins médicaux. Le Collège des médecins du Québec joue un rôle important. Il a conçu un système de surveillance et d'amélioration de la performance de ses membres basé sur la surveillance par indicateurs et par visites d'inspections professionnelles ainsi que sur des activités d'amélioration de la performance des établissements hospitaliers.

Le 7 novembre 2002, le nouveau Code de déontologie des médecins du Québec est entré officiellement en vigueur. Alors que le Code de déontologie n'avait pas été révisé depuis plus de vingt ans, l'exercice médical et son contexte ont, eux, beaucoup évolué. Le Code de déontologie tient donc compte de ces nouvelles réalités, notamment en ce qui a trait au dévoilement des accidents de soins, à l'indépendance professionnelle, à la prise en charge et au suivi des patients et aux thérapies alternatives. La relation médecin-patient se fonde sur une très grande confiance. Les patients comptent sur leur médecin pour les informer avec précision au sujet de l'évolution de leur maladie, par exemple, ou des complications survenues après une intervention. Le nouveau Code sensibilise les médecins à l'importance de dévoiler dès que possible tout incident, accident ou complication qui pourrait avoir des répercussions sur la santé de leur patient.

L'article 56, relié à la divulgation, est le suivant :

56. Le médecin doit informer, le plus tôt possible, son patient ou le représentant légal de ce dernier, de tout incident, accident ou complication susceptible d'entraîner ou ayant entraîné des conséquences significatives sur son état de santé ou son intégrité physique. 
Le code fait l'obligation d'informer la victime de ce qui s'est passé. Le code n'oblige pas le médecin à conclure sur les tenants et aboutissants d'un accident, ni à s'avouer coupable ou à identifier un coupable. «De toute évidence, cette obligation est le point de départ d'un système de gestion de risques. La reconnaissance de l'accident et sa divulgation à la personne concernée est le premier pas pour en prévenir la récurrence ${ }^{11}$.

\subsection{Le programme d'agrément et la sécurité des patients}

Ces dernières années, le Conseil Canadien d'Agrément des Services de Santé (CCASS $)^{12}$ a soulevé la question de la sécurité des services de soins et a amené les établissements hospitaliers à considérer la sécurité comme une dimension essentielle de la qualité des services de soin. Au Québec, le réseau de santé étant différent des autres provinces du Canada, le Conseil Québécois d'Agrément des Services de Santé a été créé. Complémentaire au CCASS, il a des missions spécifiques au réseau Québécois (par exemple, pour les CLSC - Centre local de services communautaires - qui n'existent qu'au Québec et les CHSLD - Centre d'hébergement et de soins de longue durée- qui n'étaient pas soumis aux normes du CCASS).

Le programme d'agrément du CCASS favorise la qualité dans les soins de santé. Le CCASS considère que les activités d'amélioration de la qualité vont aussi améliorer la sécurité des

\footnotetext{
${ }^{11}$ Mémoire déposé par le Collège des médecins en commission parlementaire dans le cadre de la loi 113.

${ }^{12}$ En tant qu'organisme non gouvernemental sans but lucratif, le CCASS a comme rôle principal d'aider les organismes de services de santé. Il examine objectivement les soins et la qualité des services prodigués aux clients de chaque organisme, en comparant les résultats à une série de normes nationales. Il offre un programme d'agrément en deux volets. Le premier volet porte sur l'auto-évaluation, c'est-à-dire que l'organisme qui demande l'agrément détermine sa propre conformité aux normes nationales. Plusieurs éléments sont examinés au cours du processus d'agrément, notamment les soins prodigués aux clients et la prestation de services, les pratiques ayant trait à la gestion de l'information et la gestion des ressources humaines, ladministration de l'organisme et la gestion de l'environnement. Des visiteurs qui ne sont pas associés à l'organisme entreprennent ensuite la visite d'agrément, en utilisant les mêmes normes nationales pour évaluer l'organisme de façon indépendante. Un résumé des conclusions de la visite est préparé sous forme de rapport, qui porte principalement sur les points forts et les faiblesses de l'organisme. Des recommandations sont formulées en vue d'aider l'organisme à élaborer des plans qui lui permettront de rectifier ses faiblesses et de maintenir ses points forts.
} 
patients. Les pratiques, les projets d'amélioration de la qualité ainsi que les activités de gestion des risques qui contribuent à maintenir et améliorer la sécurité des patients sont évalués dans le programme d'agrément. Les normes ont évolué de façon à mettre l'accent sur les processus liés aux soins des patients tels que l'administration sécuritaire des médicaments ou les processus de documentation clairs. A travers les différentes étapes du processus d'agrément, le CCASS montre qu'il est possible d'identifier les problèmes liés à la sécurité des patients et de mettre en place les mesures de préventions adaptées. Ce processus incite les établissements à mener une auto-évaluation systématique des aspects ou des processus non sécuritaires de leurs activités.. Une révision indépendante menée par des pairs de l'externe doit permettre de valider les constatations et d'émettre des recommandations pour remédier à la situation. La liste des organismes agréés au QUEBEC par le CCASS a été mise en annexe.

\subsection{L'importance de la loi 113 et les difficultés rencontrées par les établissements}

Les informations que nous avons recueillies concernant la situation actuelle au Québec nous montre bien l'importance de la loi 113. En effet, l'analyse des rapports d'incidents/accidents et les analyses approfondies ne sont pas systématiques et varient grandement d'un établissement à un autre (voir d'un service à un autre dans certains établissements). Dans le cas où l'analyse statistique des rapports d'incidents/accidents n'est pas effectuée, cela rend presque inutile la déclaration (car il n'y pas de compilation des données et aucun moyen de savoir ce qui s'est vraiment passé). De plus, pour les établissements où les rapports sont analysés, il n’y a pas de retour ou de communication des résultats de façon systématique. Certains services essentiellement les pharmacies - «publient» régulièrement les résultats mais d'autres ne semblent pas encore sensibilisés. Il va donc falloir faire évoluer les mentalités et les pratiques en matière de gestion des risques.

Même si ce n'est pas l'objet de ce rapport, nous ne pouvons pas ignorer l'environnement budgétaire et les problèmes actuels de financement dans le réseau de la santé. De nombreux établissements sont préoccupés par le manque de moyens et de ressources humaines 
disponibles pour mettre en application la loi 113. Il sera important que le ministère «aide » et « accompagne » les établissements.

\section{DEFINITIONS : SYSTEMES COMPLEXES ET DEFAILLANCES}

Nous allons tout d'abord présenter les spécificités du système de soins et nous allons définir les principaux concepts de la gestion des risques et les défaillances des systèmes complexes.

«L'activité de soins est une activité de service très particulière. Elle correspond à une production de service variable qualitativement et quantitativement nécessitant des capacités d'adaptation considérables. En effet, le système doit s'adapter à la variabilité des flux, à l'urgence et à chaque situation, le cas de chaque patient pouvant requérir des compétences multiples et spécialisées qu'il est nécessaire d'identifier et de réunir dans un lieu et un délai contraint »(ANAES, 2003).

Les événements indésirables qui se produisent lors de la prestation de soins sont le plus souvent liés à des erreurs ou sont le fait d'une inattention, d'une distraction (l'alarme d'un autre patient qui sonne), d'un faux mouvement ou d'un oubli, ou d'un événement fortuit (tel que le bris d'équipement). Il ne s'agit pas la plupart du temps d'erreurs commises par une personne en particulier (on recherche trop souvent les responsabilités individuelles) car il se peut que certains événements résultent d'un ensemble de causes (souvent apparues en cascade) ou proviennent de l'organisation des soins elle-même. Par exemple, il peut s'agir d'une mauvaise transmission d'informations entre deux services d'un hôpital (délai pour obtenir les résultats d'un test) ou au sein d'un même service (lors d'un changement de quart par exemple). Ainsi, pour maîtriser de tels événements il faut considérer non seulement les défaillances techniques et humaines mais aussi les défaillances organisationnelles aujourd'hui encore trop peu prises en compte. Les solutions trouvées dans l'industrie aéronautique et l'industrie nucléaire peuvent en grande partie être appliquées au domaine de la santé. 
Un système sûr doit permettre de résister aux erreurs et aux pannes et de se protéger contre leurs conséquences : c'est l'approche systémique de la gestion des risques.

\subsection{Le système de soins : un système complexe}

Nous allons tout d'abord mettre en évidence la complexité du système de soins. Nous allons définir successivement la notion de système et celle de système complexe.

\subsubsection{Définition d'un système}

Un SYSTÈME est un ensemble d'éléments (humain, matériel,...) interdépendants qui interagissent et concourent à un but commun. Chaque organisation a une structure de base composée d'éléments essentiels qui sont présents dans toutes les actions ou activités ou qui y contribuent : le personnel ; l'équipement et les outils ; l'environnement et l'administration.

\subsubsection{Définition d'un système complexe}

Un SYSTÈME COMPLEXE est un système constitué par une grande variété de composants ou d'éléments, possédant des fonctions spécialisées (Gell-Mann, 1995). Ces éléments peuvent être organisés en niveaux hiérarchiques internes (cellules, organes, système d'organes). Les différents niveaux et éléments individuels sont reliés par une grande variété de liaisons. Il en résulte une haute densité d'interconnexions. Les interactions entre éléments d'un système complexe sont non linéaires. L'approche systémique englobe la totalité des éléments du système étudié ainsi que leurs interactions et leurs interdépendances

\subsubsection{Le système de soins et le système « hôpital »}

Le SYSTÈME DE SOINS nécessite la réalisation de nombreuses activités et le bon fonctionnement de multiples processus. Les soins de santé sont prodigués jour et nuit, tous les 
jours de l'année dans des différents endroits (centres hospitaliers, CLSC,...). Le terme de réseau de soins utilisé au Québec est bien adapté (voir représentation graphique précédente). Ils reposent sur des techniques, compétences et savoir-faire sophistiqués (ANAES, 2003). L'amélioration des diagnostics et des traitements a complexifié les processus. D'après les définitions proposées ci-dessus, on peut considérer que la production de soins est assurée par un système complexe.

Toutefois, un hôpital peut aussi être considéré comme un système complexe. En effet, lors d'une seule hospitalisation, le patient peut voir un grand nombre d'intervenants d'une même équipe de soins ou de différents services ou départements. Au sein d'un même hôpital, de nombreuses spécialités coexistent, de nombreux services se complètent et des fonctions et spécialités différentes s'entrecroisent (Wiener, 1998). On peut représenter le «système » hôpital de la façon suivante :

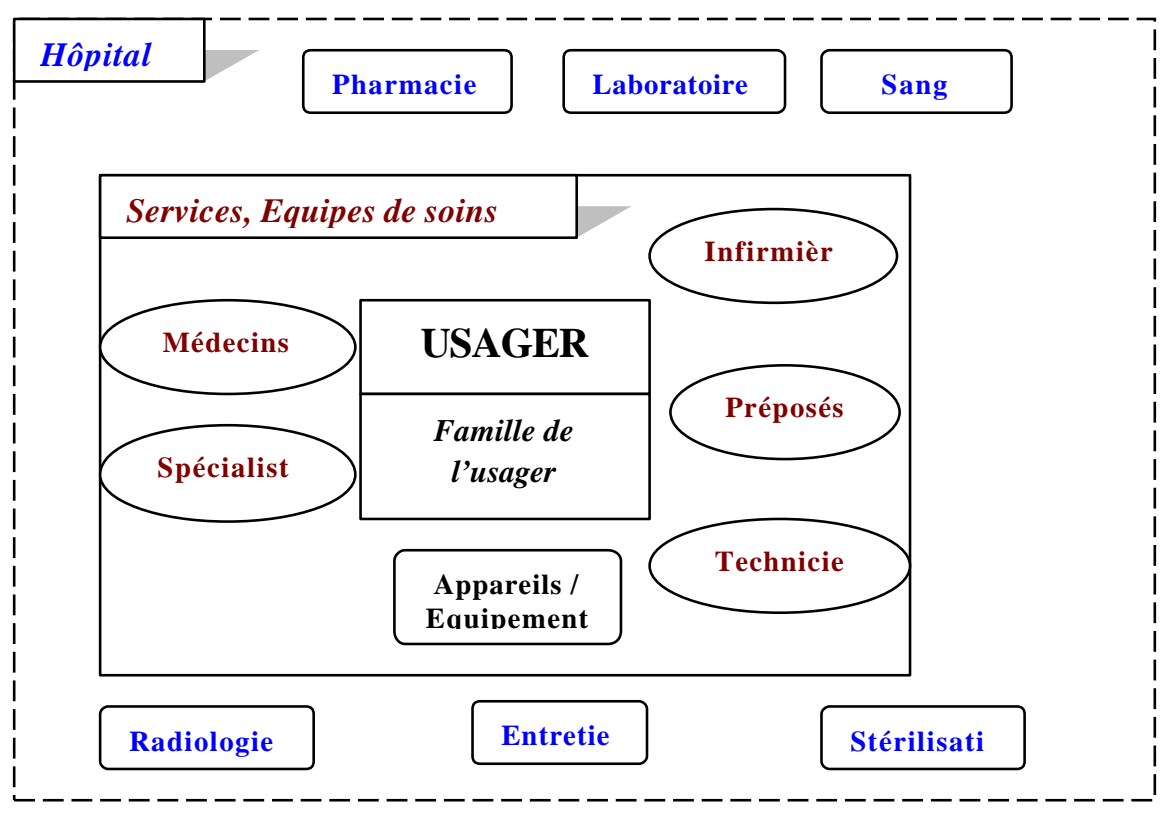

Graphique 3 : Le « système » hôpital 
De plus, un même établissement hospitalier peut avoir plusieurs sites. Une salle d'opération ou une unité d'obstétrique peuvent aussi être considérées comme un (sous)-système complexe de l'hôpital avec en plus des équipements de haute technologie.

\subsection{Les défaillances des systèmes complexes}

La compréhension des différentes causes de défaillance d'un système complexe est un préalable à la construction d'une démarche de gestion des risques (ANAES, 2003). Il y a une vaste littérature sur les défaillances des systèmes complexes. La plupart des références viennent de l'industrie (aviation et nucléaire). Les accidents industriels dans les systèmes complexes se produisent principalement par la présence de causes multiples, chacune nécessaire à la réalisation de l'événement indésirable mais qui prises indépendamment n'aurait pas toujours pu entraîner l'accident. Il s'agit la plupart du temps d'une chaîne d'événements. Les différents types de défaillances sont les défaillances techniques, humaines et organisationnelles. Pour $85 \%$ des cas d'accident, c'est le système (organisation, processus ou ressources, par exemple) qui est en cause et dans seulement $15 \%$ des cas, les accidents sont dus à une responsabilité strictement individuelle (Reason, 1993). De plus, certaines caractéristiques du système de soins ou de l'environnement du système peuvent contribuer à la survenue de l'accident (mauvaise coordination, surcharge de travail, fatigue, stress). Souvent ces vulnérabilités sont présentes dans l'organisation longtemps avant un incident ou un accident spécifique.

\subsubsection{Chaîne d'événements, causes proximales et causes racines}

Dans l'industrie aéronautique et dans l'industrie nucléaire, les nombreuses études ont conduit à une meilleure compréhension des CAUSES D'INCIDENT ET D'ACCIDENT, mettant moins l'accent sur les individus qui font des erreurs que sur les DÉFAILLANCES ORGANISATIONNELLES et sur les FACTEURS PRÉEXISTANTS. Ces études ont illustré 
la complexité de la CHAÎNE D'ÉVÉNEMENTS qui peuvent conduire à la réalisation du risque. Dans la littérature spécifique au système de soins, les chaînes d'événements et le cumul des erreurs sont souvent mis en évidence.

Prenons l'exemple d'une résidente qui a inversé deux dossiers et donne au médecin le mauvais dossier. Si le médecin ne détecte pas l'inversion, il fait une injection sans avoir connaissance que le patient en question est allergique à ce médicament. Le médecin dans ce cas particulier est l'ACTEUR de première ligne. Son erreur va conduire à la réalisation du risque. Il s'agit d'une ERREUR active.

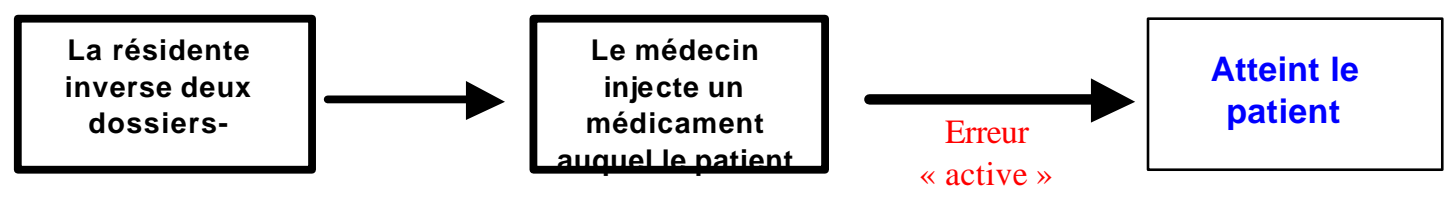

Acteur de première ligne

Graphique 4 : Exemple d'erreur active dans une chaîne d'événements

L'analyse des incidents et des accidents ne doit pas se centrer uniquement sur les erreurs des acteurs de première ligne. Elle doit chercher à identifier les insuffisances dans les défenses du système qui ont permis à l'erreur de l'acteur, à la panne ou à toute combinaison des deux, de dégénérer en accident (ANAES, 2003) et dans notre cas de toucher le patient.

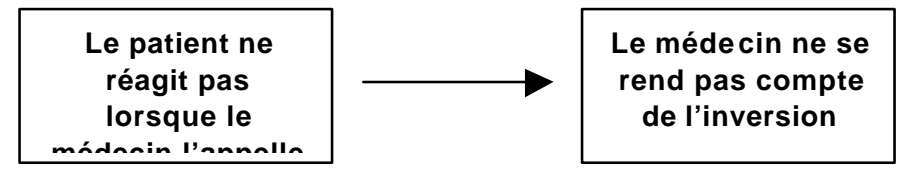




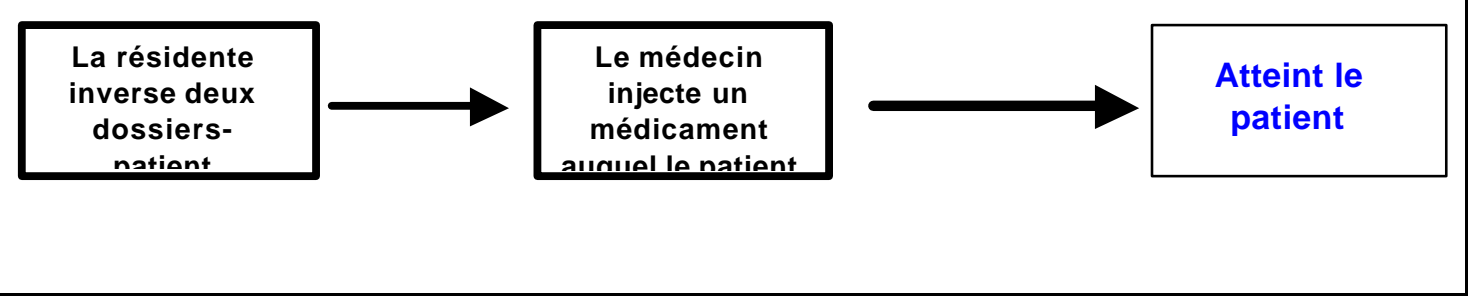

Graphique 5 : Chaîne d'événements et causes

Il est vrai que si la résidente n'avait pas inversé les dossiers, le médecin n'aurait certainement pas fait l'erreur. Dans une chaîne d'événements, chaque événement précédent est la CAUSE IMMEDIATE OU CAUSE PROXIMALE. Sans l'événement en question, l'accident ne serait pas survenu. Mais la chaîne d'événements peut être arrêtée à chaque maillon.

On peut empêcher le risque de se réaliser : on RECUPERE LA SITUATION ex ante. En effet, la résidente aurait pu s'en rendre compte et informer le médecin avant qu'il ne fasse sa prescription ou le médecin aurait pu s'en rendre compte (il y a des procédures qui permettent de vérifier l'identité d'un patient et qui servent de DEFENSES à ce type d'erreur) et ne pas faire l'injection. Les défenses peuvent être de différentes natures : physiques (alarmes,...) ou managériales (procédure de vérification, formation,...).

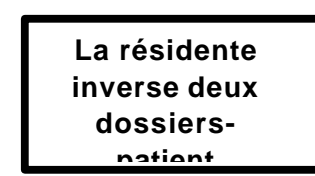

s'en aperçoit

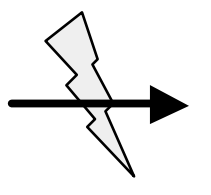

Défense

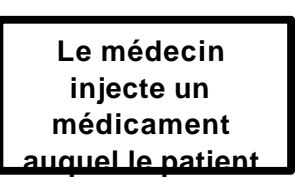

s'en aperçoit

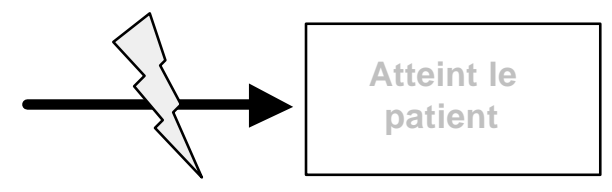

Défense

Détection /

Récupération ex ante

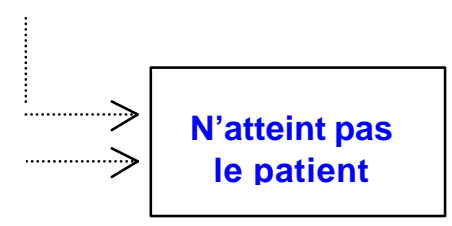

Graphique 6 : Exemples de défenses dans une chaîne d'événements 
Dans le cas ci-dessus, il y a deux causes (proximales) qui peuvent être identifiées : une erreur de la résidente et une erreur du médecin dans sa vérification (la défense qui n’a pas fonctionné). Ainsi, les situations où le risque se réalise et touche le patient sont souvent liées aux situations dans lesquelles une (ou des) erreur(s) humaine(s) a pu survenir, et, par une chaîne d'événements conduire à l'accident par défaut de défenses du système. Il ne faut d'ailleurs pas uniquement se focaliser sur les défenses de l'acteur de première ligne (même si ces défenses sont les plus importantes car elles sont les ultimes défenses à pouvoir empêcher la réalisation de l'accident) mais aussi identifier les défenses en amont de la chaîne d'événements. L'accident n'est souvent que le révélateur d'une ou plusieurs mauvaises défenses du système dans son ensemble.

L'association des pharmaciens du Québec a donné l'exemple suivant dans son mémoire rédigé dans le cadre du projet de loi 113. Un usager a reçu la bonne dose du médicament grâce à une intervention du pharmacien qui a prévenu l'accident à l'étape de la prescription. Tous les systèmes d'utilisation des médicaments prévoient des vérifications multiples par plusieurs professionnels et employés différents. Le graphique suivant reprend les différents cas pour une chaîne de 2 événements :

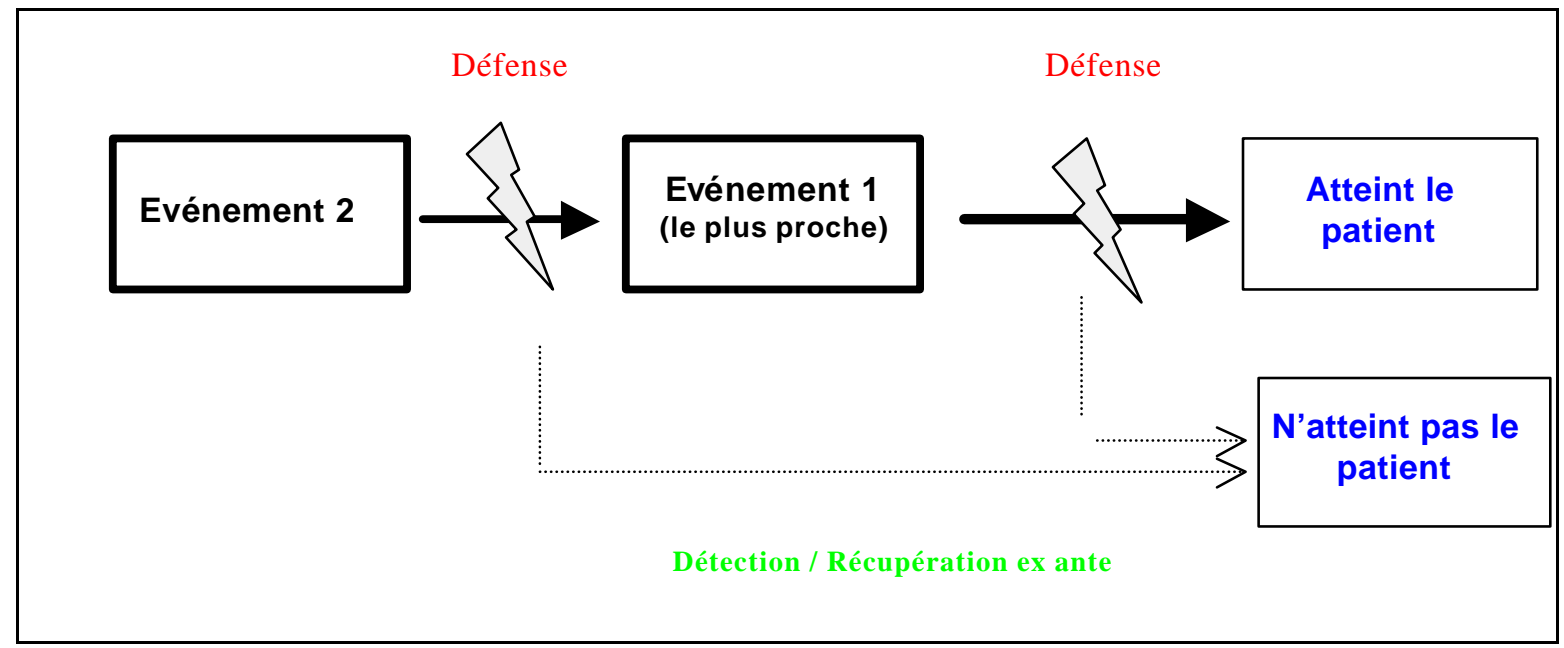

Graphique 7 : Chaîne d'événements qui va (ou non) atteindre le patient

Pour faire progresser la sécurité, il convient de considérer que l'accident n'est pas uniquement lié aux erreurs d'un individu ou d'un groupe d'individus - dans notre cas la résidente et le médecin (appelées DEFAILLANCES HUMAINES). Il peut y avoir des DÉFAILLANCES 
TECHNIQUES (un appareil qui tombe en panne, qui se dérègle,...) ou des DÉFAILLANCES ORGANISATIONNELLES (procédures erronées, mauvaise communication, délai dans l'obtention des résultats,...). Les défaillances organisationnelles vont la plupart du temps conduire à la réalisation d'erreurs humaines, il s'agira d'une des causes d'erreur. La plupart du temps cette erreur est vue comme une DEFAILLANCE ACTIVE (Reason, 1990)

De plus, tout système comporte aussi des facteurs préexistants favorisant l'erreur. Ces facteurs vont augmenter la probabilité de faire des erreurs (coordination insuffisante, surcharge de travail, fatigue, stress, mauvais design de l'appareil,...). Il s'agit de FACTEURS DE CONTEXTE ou facteurs contributifs ou encore appelés défaillances latentes (car il s'agit de facteurs «présents » dans le système et qui vont contribuer à la survenance de l'événement). L'acteur de première ligne révélera ces défaillances latentes par des erreurs actives à l'occasion de circonstances particulières. On parle aussi de CAUSES RACINES pour bien montrer qu'il s'agit de « causes » premières qui ont favorisé l'erreur (pour bien les différencier des causes proximales). Les causes racines des événements indésirables liés à la prestation des soins peuvent résider dans des facteurs tels le recours à des médecins remplaçants ou infirmières intérimaires, des problèmes de supervision, une charge de travail excessive, des insuffisances de formation initiale ou continue (Vincent et al. 2000).

Le modèle de Reason (Reason, 1990), intitulé le «Swiss cheese model» - car il utilise une représentation avec des tranches de fromage suisse - considère que les défaillances latentes représentent les trous du fromage par lesquels se sont faufilées les défaillances actives. Les défaillances actives ont des répercussions immédiates sur les systèmes et sont habituellement commises par des pilotes, des médecins, des infirmières et d'autres acteurs à « l'avant-scène ». Il s'agit d'erreurs - qu'il ne faut pas confondre avec faute (qui peut avoir un caractère intentionnel) ou de déviances - écart d'une pratique ou non-respect d'une procédure. Il peut s'agir par exemple d'une application insuffisante d'une procédure ou d'un protocole (comme le lavage des mains). Par contre, les défaillances latentes sont le fait de ceux qui se trouvent à «l'arrière-scène» du système, entre autres les gestionnaires, les décideurs et les concepteurs de 
système. Un examen de six études de cas (dont Three Mile Island, Challenger,...) permet de croire que ce sont les défaillances latentes plutôt que les défaillances actives qui menacent le plus la sécurité dans les systèmes complexes. Les défaillances latentes sont fréquemment présentes de longue date dans le système mais elles ne sont souvent pas perçues comme des défaillances potentielles car les individus sont habitués à travailler de cette façon, et elles font partie de la culture professionnelle. Elles peuvent se situer dans des décisions managériales, des procédures, des appareils (par exemple, une mauvaise ergonomie), des dispositions d'équipe, mais également dans certaines caractéristiques des patients pris en charge (Chopard et Wasserfallen, 2002). Tous les systèmes complexes contiennent de tels facteurs latents. Le modèle de Reason est utilisé comme modèle organisationnel d'accident dans le système de santé dans de nombreux pays comme l'Australie et la Grande Bretagne.

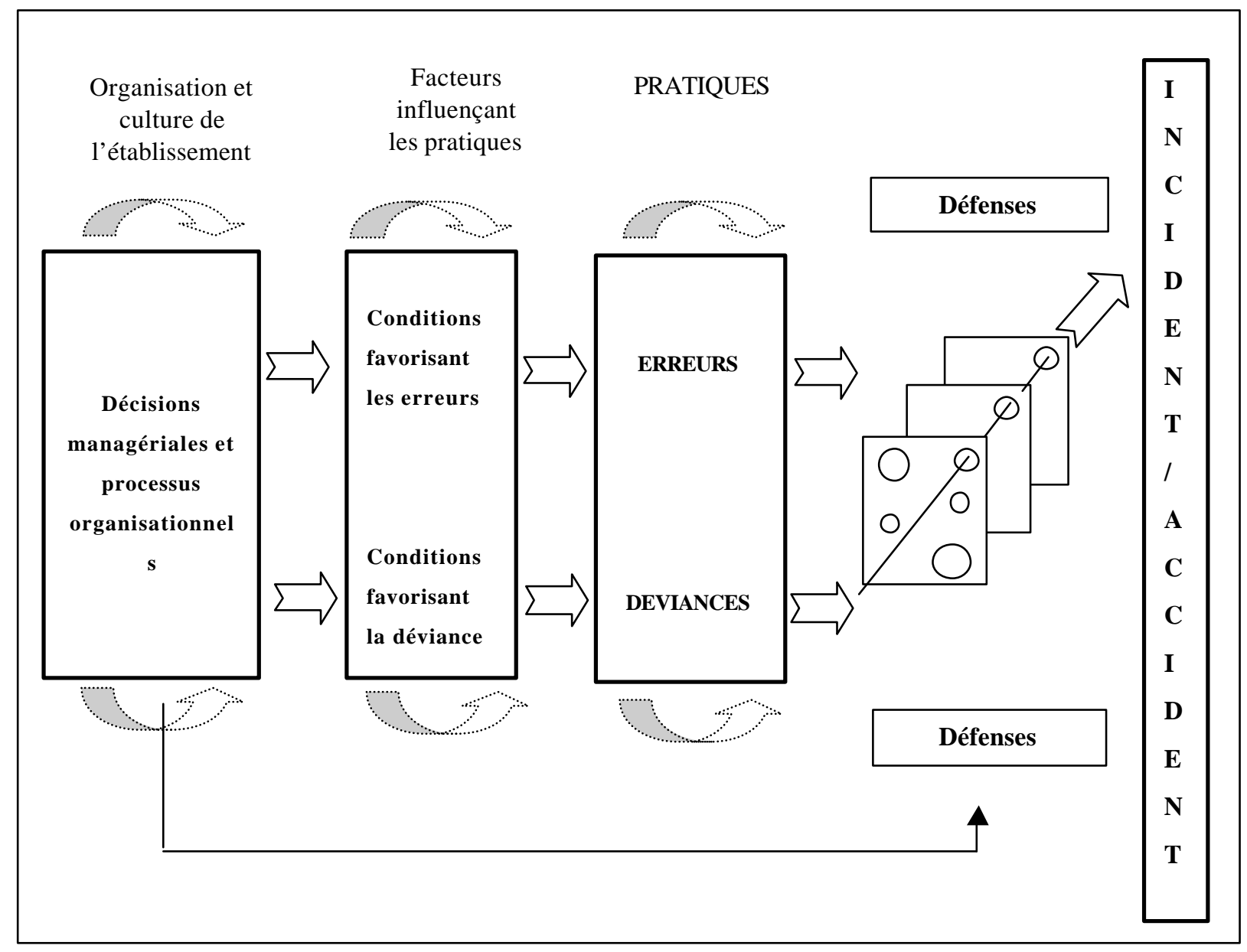

Graphique 8: Le modèle de Reason (adapté de Reason (2001) in Vincent 2001) 
La compréhension de la survenue des accidents dans un système complexe impose la prise en compte des différentes sources de défaillance, qu'elles soient latentes ou actives. Les défaillances humaines, les défaillances organisationnelles et les défaillances techniques seront étudiées successivement.

\subsubsection{Facteur Humain : erreurs humaines et conditions du système favorisant l'erreur}

Comme on l'a vu dans la section 3.1, la défaillance des systèmes complexes conduit à s'intéresser au rôle de l'erreur humaine. Perrow (1984) montre que, dans la plupart des cas, les accidents ont pour origine une ou plusieurs défaillances humaines. La littérature montre que l'analyse des accidents impute 65 à $80 \%$ des causes immédiates aux opérateurs de première ligne (Amalberti, 1996). Toutefois, les erreurs résultent la plupart du temps de systèmes mal conçus et de défenses inefficaces plutôt que de personnes négligentes ou incompétentes (IOM, 1999). Deux exemples peuvent servir d'illustration.

Prenons tout d'abord l'exemple d'une erreur liée à la médication (Voir graphique 9). Une infirmière procède à une injection par la mauvaise voie. L'infirmière s'est trompée. La cause est dans ce cas là une erreur individuelle. Toutefois, dans d'autres cas, il pourra s'agir du contenu de la seringue qui est en défaut. Mais là encore, ce sera le geste posé sur le patient par l'acteur de première ligne qui sera la cause immédiate de l'accident.

Prenons un autre exemple lié à la stérilisation (Voir graphique 10). Le médecin ou l'infirmière utilise un instrument non stérilisé (alors qu'il est censé l'être). Mais là encore, ce sera le geste posé sur le patient par l'acteur de première ligne qui sera la cause immédiate de l'accident. Il ne faut pas, dans ces deux cas, s'en tenir à l'analyse du dernier maillon de la châne (qui en contient obligatoirement plusieurs) mais chercher à savoir pourquoi cette seringue non stérilisée ou le mauvais contenu est arrivé à Punité et pourquoi le médecin ou l'infirmière qui l'a utilisé ne s'en est pas rendue compte. Nous avons représenté les deux exemples en mettant en foncé les « acteurs » ou les «services » pouvant être impliqués dans la chaîne d'événements. 


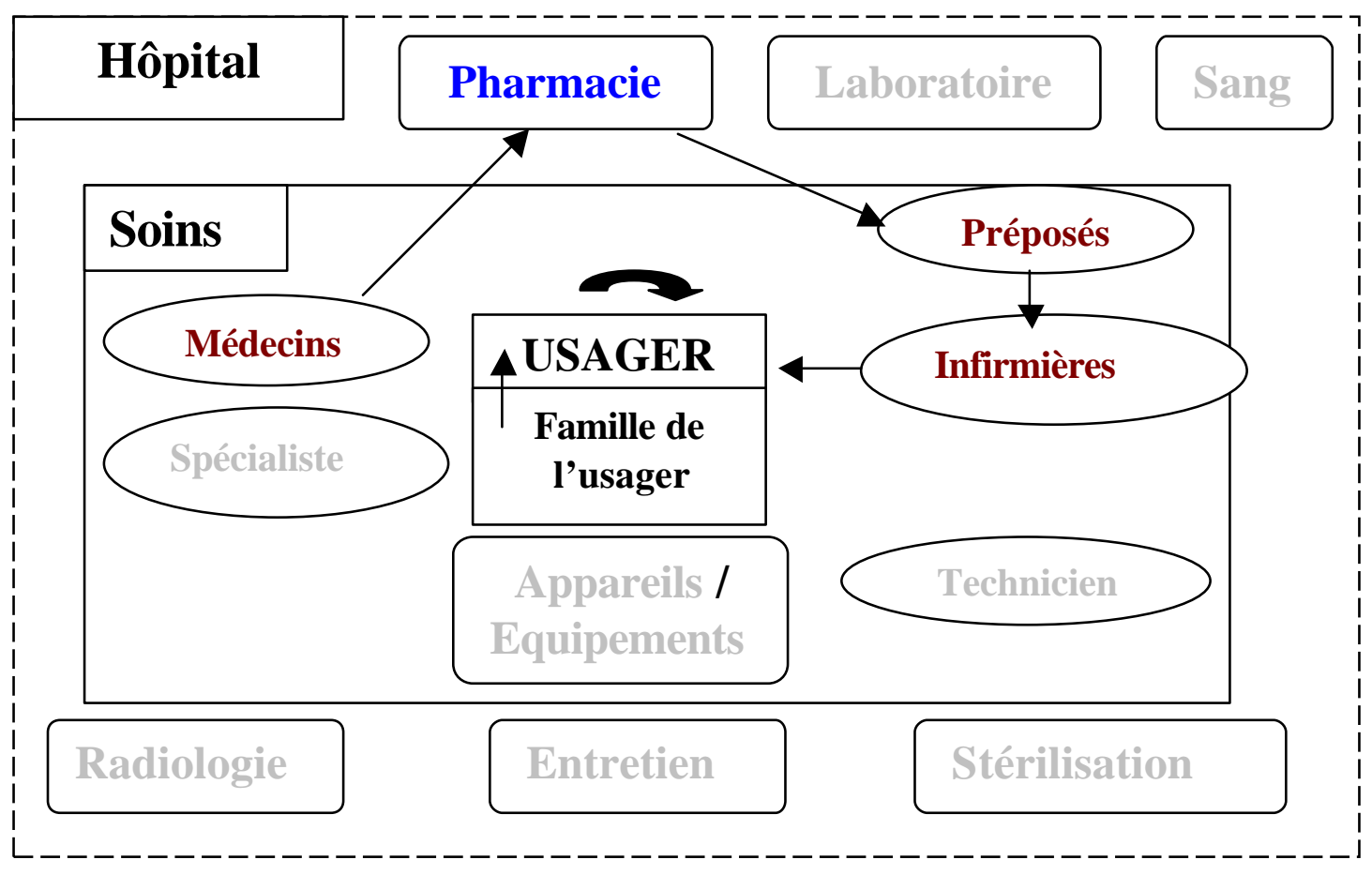

Graphique 9 : Accident lié à la médication dans le système « hôpital »

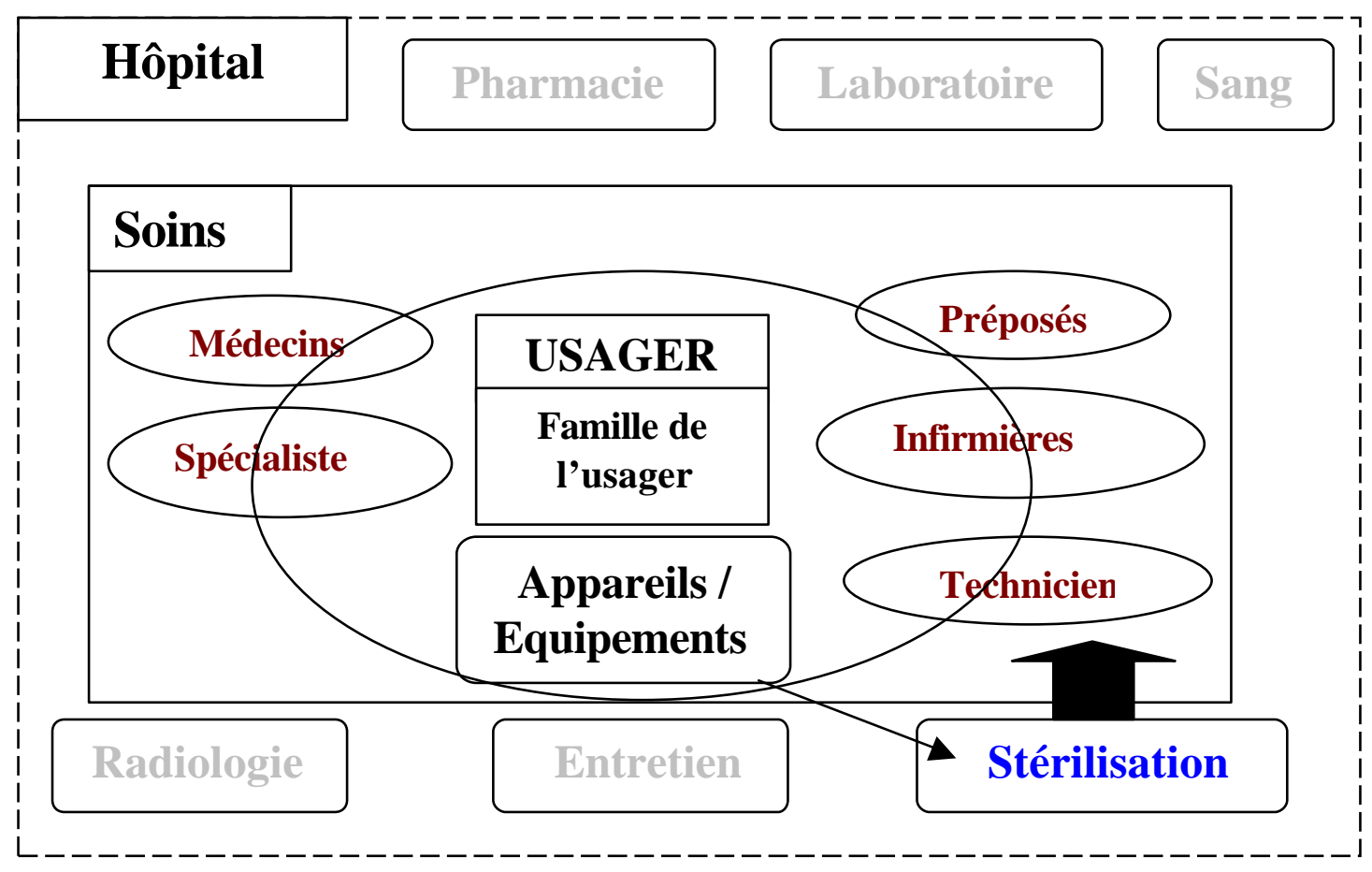




\section{Graphique 10 : Accident lié à la stérilisation dans le système « hôpital »}

L'étude de Donchin, Gopher et al (1995) qui a porté sur la nature et les causes des erreurs humaines dans une unité de soins intensifs au sein d'un hôpital universitaire a montré qu'en moyenne sur 178 activités faites par patient et par jour, il y avait 1,7 erreurs. Pour l'ensemble de l'unité de soins intensifs, une erreur grave ou potentiellement préjudiciable survenait en moyenne deux fois par jour. Les médecins commettaient autant d'erreurs que les infirmières, bien que le nombre quotidien d'activités des infirmières dépasse celui des médecins. Une bonne partie de ces erreurs sont attribuables aux problèmes de communication entre les médecins et les infirmières.

Une erreur quelle que soit sa qualification dévie de quelque chose, de quelque norme. On trouve généralement trois types d'erreurs humaines :

- ERREUR DE CONNAISSANCE (information inadéquate ou incomplète) : la personne pensait faire bien mais elle fait «mal». Cela peut être due à une mauvaise information (mauvais dossier) ou à un manque d'information (délai des résultats) ou de formation (ne connaît pas le fonctionnement de la machine).

- ERREUR DE PERFORMANCE : la personne le savait mais s'est quand même trompée. Par exemple, l'infirmière de la salle de soins intensifs qui est alertée par une alarme d'un patient alors qu'elle est au chevet d'un autre administrera en vitesse au second un médicament par la mauvaise voie (intra-artérielle plutôt qu'intraveineuse). Elle le sait mais elle s'est quand même trompée. Le manquement se situe souvent au niveau de l'exécution. Par exemple, l'infirmière remonte les côtés du lit alors que la main du patient est coincée dans la charpente métallique

- OUBLI : la personne n'a pas fait une tâche qu'elle devait faire. Par exemple, l'infirmière a oublié de donner un médicament. 
De nombreuses études ont mis en évidence les facteurs qui influencent les taux d'erreur humaine (Cooper, Newbower et al., 1978, Perrow, 1984, Reason, 1990, Almaretti, 1996, Vincent, 2002,...). Les facteurs de contexte qui ont été identifiés sont liés au :

- CONTEXTE TEMPOREL (retard, action interrompue, actions répétitives,...),

- AU CONTEXTE PERSONNEL (charge de travail, stress et fatigue, inconfort dans la situation,...),

- A L'EQUIPE ET A SA GESTION (changement de quart, absence de collaboration, problème de répartition des taches, pression sur l'équipe, manque de supervision, conflit,...),

- AUX FACTEURS ORGANISATIONNELS ENTRE UNITÉS /SERVICES/ DÉPARTEMENTS (situation de transfert d'un usager, différences dans les politiques et procédures,...),

- $\quad$ A L'ENVIRONNEMENT DE TRAVAIL (ergonomie, bruit, lumière,...),

- A LA QUALITÉ DE L'INTERFACE HOMME-MACHINE (données erronées, mauvais design de l'appareil,...),

- $\quad$ A L'USAGER (non coopératif, intervention des proches,...).

Le graphique suivant reprend l'exemple précédant d'inversion des dossiers en identifiant les facteurs de contexte : 


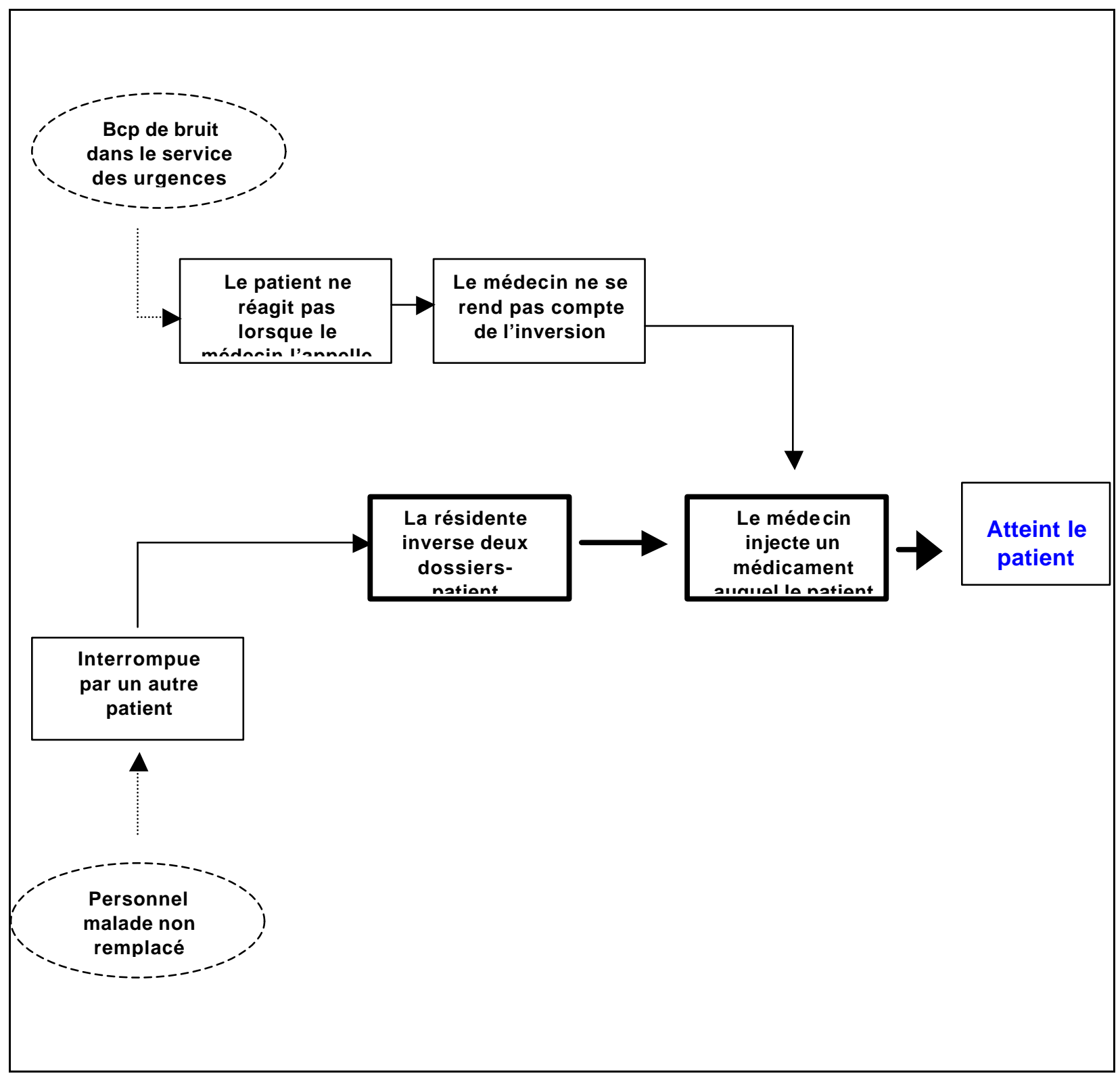

Graphique 11 : Exemple de châne d'événements, causes et facteurs de contexte

Cooper, Newbower et al. (1978) ont étudié les erreurs dans le domaine de l'anesthésie. La majorité des incidents évitables mettaient en cause l'erreur humaine. Les défaillances évidentes du matériel n'entraient en ligne de compte que dans $14 \%$ du nombre total d'incidents évitables ; en revanche, la conception du matériel était mise en cause dans de nombreuses catégories d'erreur humaine, au même titre qu'une expérience insuffisante ou une connaissance insuffisante du matériel ou de l'intervention chirurgicale pratiquée. Ces facteurs ont augmenté la probabilité 
de défaillance. Williams (1988) a évalué cette influence pour certains facteurs. Par exemple, la non-familiarité avec la tâche augmente le risque par 17 alors que la monotonie et l'ennui par 1.1. Le manque de temps pour effectuer une tâche augmente le risque 11 fois alors que le manque de sommeil 1.6 fois.

\subsubsection{Défaillances organisationnelles}

Un certain nombre d'éléments d'organisation peuvent être identifiés à la source des défaillances. La littérature impute classiquement plus de $80 \%$ (Reason, 1997) des causes d'erreurs à l'organisation avec surtout des problèmes rencontrés aux interfaces entre les acteurs ou entre les services. Lorsque l'on parle de défaillances organisationnelles, on a parfois de la difficulté à les différencier des facteurs de contexte. La plupart du temps il s'agira de causes ayant entrâné la réalisation d'erreurs humaines (mauvaise communication entre deux personnes lors d'un changement de quart,...). Sans ce maillon dans la chaîne d'événements, le risque ne se serait pas réalisé. Il peut s'agir aussi de défaillances liées à la notion de déviance comme la non application d'une procédure ou le lavage des mains sans utiliser un savon antiseptique. Il s'agit d'un mécanisme d'adaptation du système (ANAES, 2002). Les grandes catégories de défaillances organisationnelles que l'on a retenues sont :

- PROCEDURE : défaillance liée à la conception - inexistante, incomplète, ergonomie, non mise a jour - ou à l'utilisation - non utilisation, omission d'une étape, non suivi de l'ordre des étapes,...;

- COMMUNICATION : orale - non faite ou mal comprise - ou écrite - illisible, incomplet, inexacte,... ;

- DELAI : dans la provision d'un service, la prise de rendez-vous, le résultat d'un test,... 


\subsubsection{Défaillance technique (équipement/matériel)}

Le fonctionnement des établissements de santé repose de plus en plus sur des équipements techniques et matériels sophistiqués. Les défaillances peuvent concerner les appareils médicaux, les fournitures de soins, les installations matérielles et les appareils de communication.

Ces équipements peuvent connaître des défaillances se traduisant soit par une interruption de fonctionnement (panne informatique entrânant une non-disponibilité des données du patient, panne électrique, panne d'un dispositif médical), soit par des mauvais fonctionnements (résultat erroné fourni par un appareil déréglé, mauvais fonctionnement du thermostat d'une couveuse, modification du débit d'une seringue électrique) (ANAES, 2003), soit par un état particulier (mauvaise stérilisation, plancher glissant, encombré, pas propre,...) ou soit par une non disponibilité du matériel.

Des mesures visant à supprimer ou à réduire ce risque ou à s'en protéger sont à prendre : maintenance préventive et curative, formation des professionnels, rédaction de notices d'utilisation, doublement de certains équipements, élaboration de procédures de fonctionnement en mode dégradé, mise en place d'alarmes, réalisation de contrôles qualité, validation des résultats, contrôle des matériels, etc. 


\section{VERS UN MODĖLE D'ANALYSE APPROFONDIE DE CERTAINS INCIDENTS ET ACCIDENTS LIÉS À LA PRESTATION DES SOINS DE SANTÉ}

Les théories qui sous-tendent l'élaboration de la grille d'analyse CIRANO proviennent de la recherche dans l'industrie nucléaire, plus spécifiquement les analyses organisationnelles et les études liées à la «fiabilité humaine». Dans l'industrie nucléaire, comme dans l'aviation ou l'industrie pétrolière, l'enquête formalisée des incidents et des accidents est une pratique bien implantée. Dans ces domaines, les analyses approfondies ont conduit à une meilleure compréhension des causes, en mettant moins l'accent sur les individus qui font des erreurs que sur les facteurs organisationnels préexistants mais aussi sur la performance des équipes dans la gestion des incidents (et ainsi éviter que le risque se réalise) et des accidents (lorsque le risque se réalise). Ces études ont également illustré la complexité de la chaine d'événements qui peuvent conduire à la réalisation du risque.

Pour les événements indésirables qui surviennent dans les établissements de santé, le développement de stratégies de prévention à partir de telles analyses n'est pas encore complètement exploité (Vincent et al., 2000). Il existe une vaste littérature sur les analyses des « root causes» qui permettent d'identifier les causes racines. Ces méthodes sont souvent structurées selon le modèle de Reason (Vincent et al., 2000 ; JCAHO, 2001 ; Chopard et Mabboux, 2001). Mais ces méthodes ne permettent pas toujours d'analyser de façon dynamique la phase de gestion de l'incident (récupération de la situation) et très rarement l'après-accident (récupération ex-post). Par exemple, les délais de latence ou de récupération ne sont pas identifiés.

En nous basant sur le modèle développé par l'Institut de Surete et de Protection Nucléaire (IPSN) en France, nous avons cherché à proposer un cadre conceptuel qui permette d'effectuer une analyse organisationnelle de l'incident/accident qui comprenne à la fois une 
analyse approfondie des causes racines mais aussi une analyse de la gestion de l'incident ou de l'accident. Nous allons tout d'abord présenter le modèle RECUPERARE (Baumont et al. 1999) puis nous allons expliquer comment nous l'avons adapté aux établissements de soins.

\subsection{Présentation du modèle «RECUPERARE » utilisé dans l'industrie nucléaire et de la méthode d'analyse associée à ce modèle}

L'Institut de Protection et Sûreté Nucléaire a mis au point plusieurs méthodes de retour d'expérience ${ }^{13}$ pour analyser les incidents significatifs déclarés par Electricité de France (EdF) et dégager des enseignements pour la sûreté. L'une de ces méthodes appelée RECUPERARE a été développée plus spécifiquement dans le cadre des études liées à la «fiabilité humaine » (Baumont et al., 1999, 2000) Ce modèle, associant les facteurs humains et organisationnels aux aspects techniques, décrit le processus mis en jeu dans les incidents en se focalisant sur le couple défaut-récupération donc il décrit les liaisons. Ces défauts peuvent être des défaillances techniques, humaines ou organisationnelles.

Dans la chronologie qui décrit l'ensemble de l'histoire de l'incident, la phase capitale du modèle est la façon dont on a géré l'erreur ou le défaut. Comment l'a-t-on détecté ?, comment a-t-on établi le diagnostic ? comment (qui, par quel moyen) l'a-t-on "récupéré" ? et surtout en combien de temps ? L'originalité du modèle RECUPERARE est de mettre en évidence les délais de latence, de détection et de récupération, qui sont regroupés sous le terme performance. L'hypothèse principale est que l'importance des conséquences n'est pas seulement en lien direct avec les causes de l'erreur ou du défaut, mais avec les capacités de récupération de la personne, de l'équipe, c'est-à-dire du système

\footnotetext{
13 La démarche de "retour d'expérience" consiste à se mettre "à l'écoute des signaux provenant de l'installation" et à se saisir de tout accident, incident, anomalie pour en retirer le maximum d'enseignements Cette démarche a pour objet de se saisir de ces éléments anormaux sortant du cadre du prescrit pour, par une analyse détaillée, en rechercher les causes et définir les corrections propres à éviter qu'ils se reproduisent. Il s'agit d'une démarche organisée et systématique pour analyser toutes les anomalies, tous les incidents et accidents constatés, en rechercher les causes et les enchaînements, en retirer les divers enseignements, définir les mesures de correction et amélioration et assurer l'information pertinente aux parties intéressées (Verot, 1999).
} 
technique et humain. Cette phase doit donc faire l'objet d'études précises car des améliorations peuvent être apportées dans ce domaine tout autant que dans la réduction des causes à l'origine des problèmes. La méthode d'analyse associée à ce modèle consiste d'abord en une lecture systématique et structurée des compte-rendus dincidents significatifs (transmis par $\mathrm{EdF}$ ) et l'enregistrement des données pertinentes dans une base informatisée. Les données obtenues sont traitées à l'aide de dépouillements statistiques.

\subsubsection{Présentation générale du modèle RECUPERARE (Baumont et al., 1999, 2000)}

Pour modéliser les situations d'incident, les auteurs ont dherché à délimiter précisément les situations dans le temps (début et fin de l'incident, différentes phases,...), à choisir des paramètres pertinents pour l'analyse des incidents, à les disposer selon une logique qui permette une description rigoureuse de la situation et de trouver des critères de classement. Le schéma ci-dessous présente un aperçu «statique » du modèle :

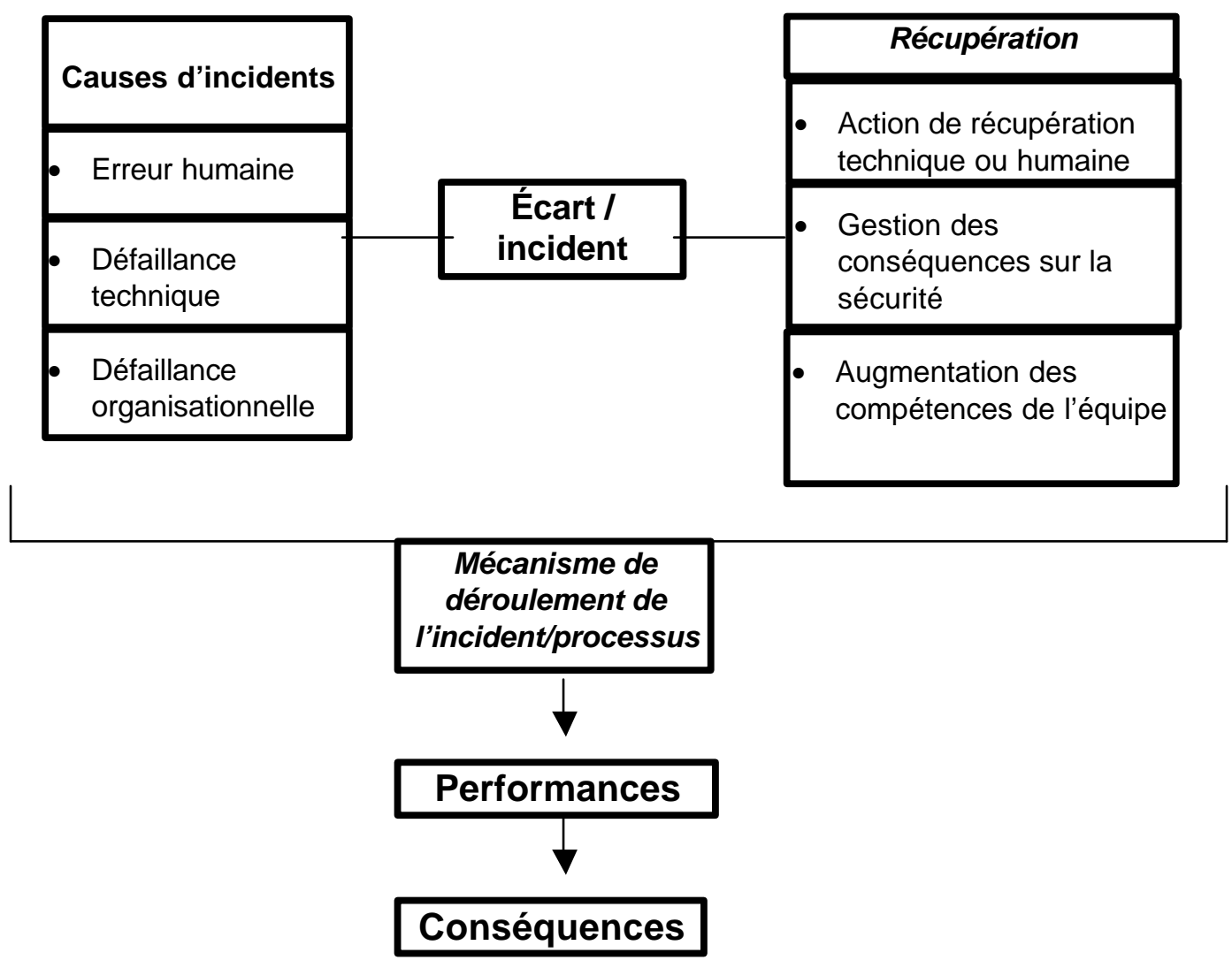

Graphique 12 : Le modèle RECUPERARE-NUCLEAIRE (Baumont et al., 1999) 
La version «dynamique » du modèle d'incident RECUPERARE permet de définir différents délais qui caractérisent le processus de façon chronologique, en prenant en compte l'instant d'apparition du défaut. Il faut noter que ce modèle met en place une chronologie précise de la récupération au sens large (ou «gestion de la situation -incident ou accident ») mais ne décrit pas la chronologie de l'apparition de toutes les causes et facteurs de contextes, ni de celle des conséquences.

\subsubsection{Les spécificités du modèle RECUPERARE :}

Basé sur une description systémique, le modèle examine l'émergence des causes de l'incident (défaillances techniques, erreurs humaines ou défaillances organisationnelles), les caractéristiques du déroulement de lincident et sa récupération (qui repose sur le système humain et les automatismes de sauvegarde, autant pour récupérer les erreurs humaines que les défaillances techniques ou organisationnelles).

Les causes de l'incident peuvent être des défaillances techniques, des erreurs humaines ou des défaillances organisationnelles. Ces trois types de défaillance sont regroupés sous le terme DEFAUT. Le modèle met en évidence les facteurs de contexte afin de pouvoir affiner l'analyse. Par exemple, pour les erreurs humaines cela permet d'identifier les facteurs de contexte à l'origine des erreurs. L'erreur est caractérisée selon la nature du geste erroné dans le contexte de travail, le mode d'utilisation des procédures liées à l'activité et leur nature d'un point de vue cognitif.

La récupération peut être décrite comme la conjugaison de trois types d'activités liées à la gestion de l'incident : la correction des défauts, la gestion des conséquences réelles et potentielles liées à la sûreté en utilisant éventuellement les procédures, la mobilisation des ressources humaines que requiert la situation, tout en leur fournissant l'information nécessaire. 
Ces trois activités se complètent et les délais pour récupérer la situation renseignent de manière synthétique sur le temps nécessaire pour gérer l'incident.

Le processus est le mécanisme de déroulement de l'incident qui évoque «ce qui s'est passé » entre le moment ou le défaut apparaît et la fin de la récupération en terme de facteurs temporels et de facteurs de dépendance. Ces facteurs sont les suivants :

- Facteurs temporels : Certaines phases du processus se déroulent avec une certaine durée (par exemple, les délais pour détecter le problème et identifier la situation). Afin de caractériser la performance humaine et organisationnelle de la gestion de l'incident, on note les différents délais qui correspondent aux différentes phases de la récupération de la situation. Ces délais peuvent devenir des indicateurs globaux de performance.

- Facteurs de dépendance : Le modèle prend en compte les dépendances entre activités, entre équipes et entre acteurs (les acteurs qui ont détecté sont-ils les mêmes que ceux qui ont récupéré la situation? Même type d'acteur dans une autre équipe, autres fonctions ?).

- Mise en service d'un système de sauvegarde ou de protection : Le modèle permet également de mettre en évidence les processus au cours desquels la récupération est effectuée par la mise en service automatique d'un système de sauvegarde.

Lors de l'étude du déroulement de l'incident dans le temps, le modèle introduit un point de référence temporelle : la mise en service du système concerné par le défaut. C'est le point capital de ce modèle dynamique qui permet une mesure de la durée des différentes phases de l'incident et conduit à une classification des incidents. Trois catégories ont été ainsi identifiées 
selon l'instant d'émergence du défaut-source et celui de sa récupération par rapport à la mise en service (ou en exploitation) du circuit concerné.

- Le couple défaut/récupération de type $\boldsymbol{A}$ considère un défaut latent, qui affecte un système ne fonctionnant pas (ou non utilisé), et récupéré avant la mise en service de ce système. La récupération de tels incidents peut témoigner de la capacité de l'organisation (procédures, hommes, contextes) à détecter un défaut latent et à le récupérer avant qu'il ne perturbe le fonctionnement ultérieur des circuits.

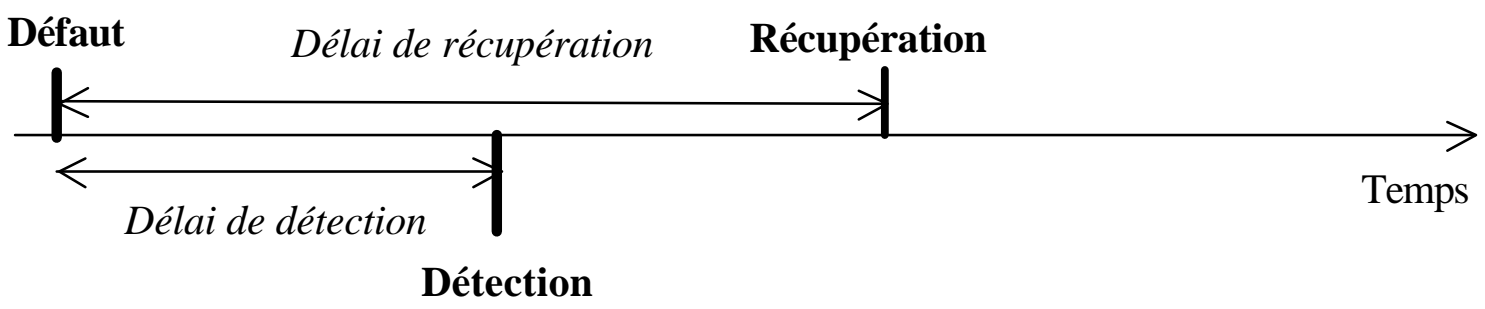

Graphique 13 : Les différents délais pour le type A

- Le Couple défaut/récupération de type $\boldsymbol{B}$ : un défaut latent qui affecte un système, est récupéré après la mise en service de ce système. La récupération de tels incidents peut témoigner de la capacité de l'organisation et des opérateurs à détecter un dysfonctionnement lors de la mise en service d'un système, en contrôlant son fonctionnement à la mise en service. Ce type d'incident est en quelque sorte l'expression de l'échec des capacités organisationnelles révélées par les récupérations des incidents de type A.

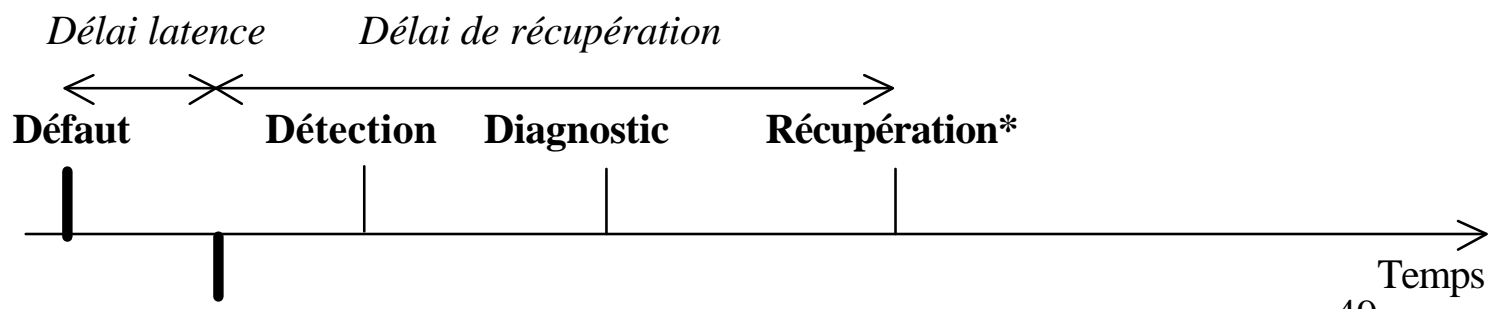




\section{Sollicitation \\ système}

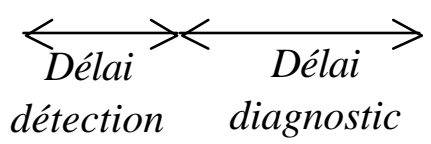

Graphique 14 : Les différents délais pour le type B

- Le Couple défaut/récupération de type $C$ : un défaut (intempestif ou non) affecte un système alors que celui-ci est en cours d'exploitation (ici la récupération se situe, bien sûr, après la mise en service du système concerné). La récupération de tels incidents témoigne de la capacité de l'organisation à détecter un dysfonctionnement dans les activités en cours (sa capacité d'auto contrôle).

Remarques :

(1) Il n'est pas toujours aisé de déterminer les délais de récupération d'un incident ${ }^{14}$. Il y a différentes "fins" pour un incident. L'IPSN distingue en particulier deux délais de récupération. L'un concerne le délai entre le défaut et le moment où la situation est redevenue conforme aux critères de sûreté (état sûr ou retour dans le domaine de fonctionnement) souvent indiqué comme "fin" de lincident dans les comptes rendus d'incidents, l'autre entre le défaut et le moment où les composants défectueux de l'installation ont été réparés. Ces délais n'ont rien à voir avec un retour à la disponibilité initiale. Le premier délai correspond à la capacité à gérer la sûreté de l'installation, le second est lié à la capacité de réponse en temps réel en terme de maintenance des composants importants pour la sûreté de l'installation.

14 Ces remarques ont été faites par les auteurs du modèle RECUPERARE pour les incidents qui se produisent dans l'industrie nucléaire mais elles peuvent s'appliquer au contexte de soins. 
(2) Ce modèle permet d'examiner la conjugaison des aspects techniques et humains. Par exemple, une erreur humaine pourra être récupérée par une mise en service automatique d'un système de sauvegarde ou encore une défaillance technique pourra faire l'objet d'un processus complexe de récupération par les équipes. Si la situation est récupérée par le démarrage automatique d'un système de sauvegarde, le délai entre le défaut et cette mise en service est enregistré dans la base. Le délai de détection de la situation par les opérateurs est souvent concomitant à cette mise en service automatique signalée par de nombreux signaux et alarmes. Par contre, le diagnostic de la cause à l'origine de la situation peut se révéler long.

(3) Un autre type d'incident est pris en compte : les incidents de type «organisationnel» qui ne relatent aucun défaut effectif sur l'installation elle-même mais ils mettent en cause un processus organisationnel.

Les conséquences de l'erreur peuvent apparaître dans les minutes qui suivent (comme dans l'industrie aéronautique), avoir un développement lent (comme dans l'industrie nucléaire) ou un développement très lent. Plus le délai est important et plus il est difficile d'identifier les causes exactes de l'incident.

\subsubsection{La méthode d'analyse des incidents à partir du modèle RECUPERARE}

La méthode, utilisée par l'IPSN, consiste à analyser de façon exhaustive tous les incidents

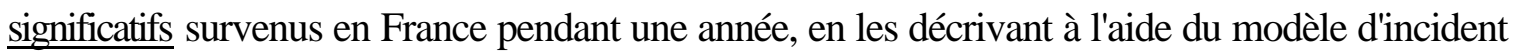
présenté dans les paragraphes précédents. Le premier objectif de cette méthode d'analyse est d'obtenir une description statistique fine des incidents significatifs d'une année, suivant des descriptifs variés, principalement liés au couple défaut/récupération, et comparables à ceux de l'année précédente. Le deuxième objectif est d'observer des délais pertinents (définis plus haut) utilisés pour évaluer la performance des sites ou de la tranche face à certains types de situation. 
La méthode d'analyse associée à ce modèle consiste d'abord en une lecture systématique et structurée des comptes-rendus d'incidents significatifs (transmis par EdF) et l'enregistrement des données pertinentes dans une base informatisée. Les compte-rendus d'incidents comprennent déjà une enquête faite par celui qui l'a rempli. La méthode d'analyse RECUPERARE est utilisée comme une grille de lecture systématique et structurée de chaque compte-rendu. L'outil nécessaire à cette analyse est une base de données informatique, dont les champs ont été élaborés à partir des descriptifs du modèle d'incident. Toutes les données concernant les incidents sont issues des comptes-rendus d'incidents significatifs transmis par les exploitants. Les données obtenues sont traitées à l'aide de dépouillements statistiques.

\subsection{Adaptation du modèle RECUPERARE au système de soins}

Conscients des différences qui peuvent exister entre le domaine de la santé et le domaine du nucléaire, nous avons essayé de construire un outil d'analyse s'inspirant de la méthode RECUPERARE mais adapté au système de soins et aux spécificités de la sécurité des patients.

Ce qui nous a séduit dans le modèle RECUPERARE, c'est d'une part l'aspect organisationnel de l'analyse mais aussi l'aspect dynamique de la gestion de l'incident et l'évaluation de la performance dans la gestion de l'incident. De plus, l'hypothèse principale du modèle qui considère que l'importance des conséquences n'est pas seulement en lien direct avec les causes de l'erreur ou du défaut, mais aussi avec les capacités de récupération du système technique et humain est primordiale dans le domaine des soins.

Sur le plan de la terminologie, il s'agit d'un modèle d'analyse des incidents significatifs. Dans notre contexte, il pourra s'agir de certains incidents et accidents. De plus, dans notre cadre d'analyse, le patient «complique » la relation Homme-Machine spécifique à l'industrie. Il y a ici une relation «Hommes-Patient-Machines » et les caractéristiques du patient peuvent jouer un rôle aggravant (comme par exemple, la situation préalable concernant son état, sa capacité de communication,... ). 


\subsubsection{Les fondements du modèle RECUPERARE-SANTE}

Le modèle RECUPERARE met en place une chronologie précise de la récupération au sens large (à partir du moment où on détecte le défaut) mais ne décrit pas la chronologie de l'apparition de toutes les causes et facteurs de contextes, ni de celle des conséquences. C'est un des éléments du modèle que nous avons un peu modifié. Dans notre cas cela peut être pertinent d'identifier la séquence des événements car cela peut renseigner la situation : par exemple, le circuit du médicament dans l'établissement ou les différentes interventions auprès du malade qui se font dans un ordre précis. Dans la méthode d'analyse, nous proposerons tout d'abord reconstruire la séquence des événements jusqu'à ce que le défaut soit détecté puis récupérer avant de toucher le patient ou dans l'autre cas, jusqu'à ce que cela atteigne le patient (on pourra chercher à identifier dans ces cas là, les défenses qui n’ont pas fonctionné).

Il est important de noter que dans certains cas où le patient a été touché, on ne peut rien faire pour récupérer la situation. Le fait même d'avoir touché le patient a des conséquences irréversibles, le décès du patient étant le cas extrême. Dans ces cas précis, la récupération ex ante et les mécanismes de défenses en place sont primordiaux.

\subsubsection{Le modèle RECUPERARE-SANTE}

Basé sur une description systémique, le modèle RECUPERARE - SANTE adapté par le CIRANO examine l'émergence des causes de l'incident/accident (défaillances techniques, erreurs humaines ou défaillances organisationnelles), les caractéristiques du déroulement de l'incident/accident et la récupération qui peut être ex ante dans le cas où cela touche le patient et ex post si cela ne touche pas le patient. Nous avons représenté en annexe les différentes composantes du modèle (voir annexe). Nous allons tout d'abord les préciser.

\section{- Les généralités :}




\section{- Informations sur l'établissement ;}

- Informations sur l'usager;

- Contexte général : il s'agit des informations qui se trouvent sur le rapport d'incident/accident AH-223 (ou autre rapport maison) concernant l'endroit où est survenu l'incident ou l'accident, l'objet (à quoi l'incident ou l'accident est relié : services cliniques/soins, médication, chute, équipement/matériel, divers, la description des faits, sans jugement, ni analyse, la situation préalable (état de la personne, capacité de déplacement, surveillance requise, considérations cliniques, environnement et lit), les mesures prises (description des gestes posés ainsi que les personnes avisées), le diagnostic consécutif versé au dossier par le médecin, les témoins identifiés, si une plainte est prévisible, avis ou recommandations.

- Informations complémentaires : il s'agit d'informations qui ne sont pas sur le AH-223 mais qui pourraient aider à l'analyse (situation et contexte avant et au moment de l'incident : capacité de communication de l'usager, accompagnement d'un proche, raison de la présence à l'hôpital, implication d'un(des) autre(s) service(s), acteurs impliqués dans l'identification/détection et moyens utilisés, gestes posés, gestion des ressources, mesures immédiates prises pour éviter la répétition de l'incident/accident,...).

- Les causes de l'incident/accident peuvent être des :

- Défaillances humaines (erreur de connaissance, erreur de performance, oubli),

- Défaillances organisationnelles (procédure, problème de communication, délai).

- Défaillances techniques (concernant les appareils médicaux - mauvais fonctionnement d'une machine, disponibilité, stérilité -, les fournitures de soins, 
les installations matérielles - plancher glissant,... -, les appareils de communication - ligne occupée, réseau non disponible,...-),

\section{- Les facteurs de contexte :}

Les facteurs de contexte vont permettre d'affiner l'analyse des causes. Certains facteurs vont influencer la réalisation de l'erreur ou de la défaillance comme des facteurs reliés au contexte temporel (contrainte de temps, action interrompue,...), au contexte personnel (charge de travail, stress,...), à l'équipe et à sa gestion (changement de quart, absence de collaboration, problème de répartition des tâches,...), à l'organisation entre unités/services/départements (transfert d'un usager de façon temporaire pour un test, changement de service,...), à l'environnement de travail (ergonomie, bruit, circulation,...) et à la qualité interface homme-machine.

- La récupération peut être décrite ici comme la conjugaison de trois types d'activités liées à la gestion de l'incident et de l'accident :

- les gestes posés pour la correction des défauts ex ante : changement de matériel, nouvelle préparation du médicament, réattribution des dossiers aux bons patients, ...),

- les gestes posés lorsque cela touche le patient (en utilisant éventuellement des procédures) : mesures prises et gestes posés - surveillance augmentée, test diagnostic, changement de médication, administration d'un antidote ou d'un médicament de façon temporaire pour stabiliser la situation, transfert dans un autre service, dans un autre établissement,...,

- la mobilisation des ressources humaines (que requiert la situation, tout en leur fournissant l'information nécessaire): appel du médecin, implication de nouveaux intervenants, spécialistes,... 
Ces trois activités se complètent ${ }^{15}$ et les délais pour récupérer la situation renseignent de manière synthétique sur le temps nécessaire pour gérer l'incident ou gérer l'accident. Il s'agit en fait d'une PHASE DE RÉCUPÉRATION qui débute dès les premiers gestes posés (dans les cas où cela atteint le patient, on pourrait parler d'une première étape de STABILISATION de la situation). Il est vrai qu'un patient transféré aux soins intensifs aura déjà eu des premiers gestes pour stabiliser la situation.

- Le processus est le mécanisme de déroulement de l'incident/accident qui évoque «ce qui s'est passé » entre le moment où le défaut apparaît et la fin de la récupération (ex ante ou ex post selon la situation) en terme de facteurs temporels (i) et de facteurs de dépendance (ii).

(i) Facteurs temporels : Certaines phases du processus se déroulent avec une certaine durée (par exemple, les délais pour détecter le problème et identifier la situation). Afin de caractériser la performance humaine et organisationnelle de la gestion de l'incident, on note les différents délais qui correspondent aux différentes phases de la récupération de la situation. Ces délais peuvent devenir des indicateurs globaux de performance.

On a noté dans le modèle RECUPERARE original l'introduction d'un point de référence temporelle (la mise en service du système concerné par le défaut). Les auteurs suggèrent que la transposition du modèle dans une autre industrie ou pour un autre type de processus doit passer par une réflexion sur le choix de ce point de référence temporelle et sur les phases de l'activité décrites. Dans notre cas, la situation peut être récupérée avant que cela atteigne le patient ou après. Dès que le patient est touché, il peut y avoir une récupération ex post de la situation. Les intervenants peuvent poser des gestes qui vont «annuler» les effets potentiels, par exemple en

\footnotetext{
${ }^{15}$ Dans le cas ou le patient n'est pas atteint, le second point ne sera pas pertinent.
} 
administrant un antidote. Dans ce cas là l'aspect dynamique du modèle est pertinent car la prise en compte des délais de détection, d'identification et de récupération est primordiale.

- Le type $A$ «n'atteint pas le patient » : considère un défaut latent qui est récupéré avant d'atteindre le patient. La récupération de tels incidents peut témoigner de la capacité de l'organisation (procédures, hommes, contextes) à détecter un défaut latent et à le récupérer avant qu'il n'atteigne le patient.

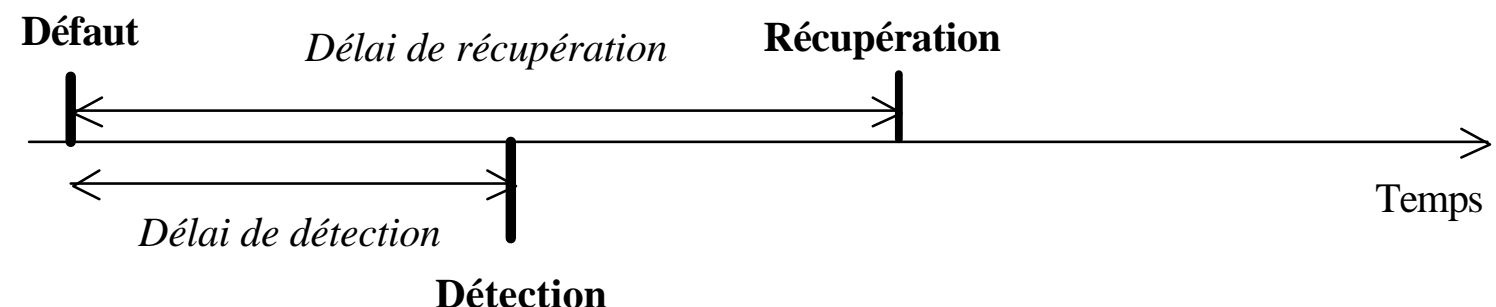

Graphique 15 : Les différents délais dans le cas où cela n'atteint pas le patient

Les moyens utilisés pour la détection peuvent influencer la performance de la récupération. Par exemple, l'alarme d'un moniteur ou l'observation de la variation des signes vitaux influencent différemment les délais de détection. Les procédures utilisées pour la détection devront être identifiées. Il peut s'agir de procédures de vérification. Par exemple, un médecin qui a en main le dossier d'un nouveau patient qu'il ne connaît pas doit s'assurer qu'il s'agisse bien du bon dossier. Il peut commencer par dire «Bonjour Monsieur X», puis si le patient ne réagit pas (il peut s'agir d'un homonyme... ou d'une personne pas capable de communiquer), continuer à vérifier un ou deux autres points. Dans tous les cas, ces procédures servent de DEFENSES. 
- Le type B «atteint le patient»: considère un défaut latent qui est récupéré après avoir atteint le patient. La récupération peut témoigner de la capacité de l'organisation et des acteurs à détecter un problème. Cela est en quelque sorte l'expression de l'échec des capacités organisationnelles révélées par les récupérations des incidents de type A. Il va falloir dans certains cas identifier le problème. Un patient trouvé par terre a très certainement fait une chute. Dans ce cas précis, on détecte et on identifie en même temps. Par contre, un patient dont les signes vitaux varient va alarmer le personnel mais il n'est pas évident que l'identification de la situation soit immédiate. Nous avons identifié 4 types de situation :

\section{$\underline{\text { Situation B1 }}$}

La situation B1 représente la séquence de référence où toutes les phases sont distinctes. La chaine d'événements atteint le patient. Il y a un certain délai avant que l'on détecte le problème, puis il y a un autre délai avant le moment de l'identification et puis il y encore un délai avant le début de la phase de récupération. Chacun des délais peut se mesurer en minutes, heures, jours.

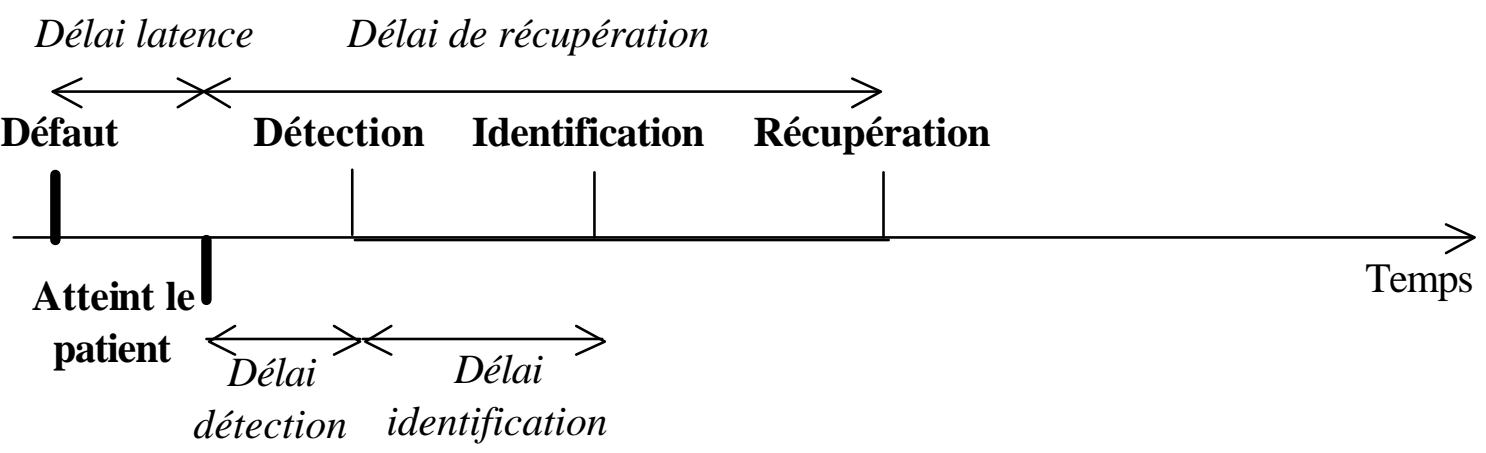


Graphique 16 : Les différents délais dans le cas où cela atteint le patient (cas 1)

\section{Situation B2}

La situation B2 représente le cas où le moment de la détection est aussi celui de l'identification. Par exemple, dans le cas des chutes, les patients sont la plupart du temps trouvés par terre. Au moment où l'infirmière s'en rend compte (et détecte), elle identifie le problème. Cela devrait réduire le délai de récupération.

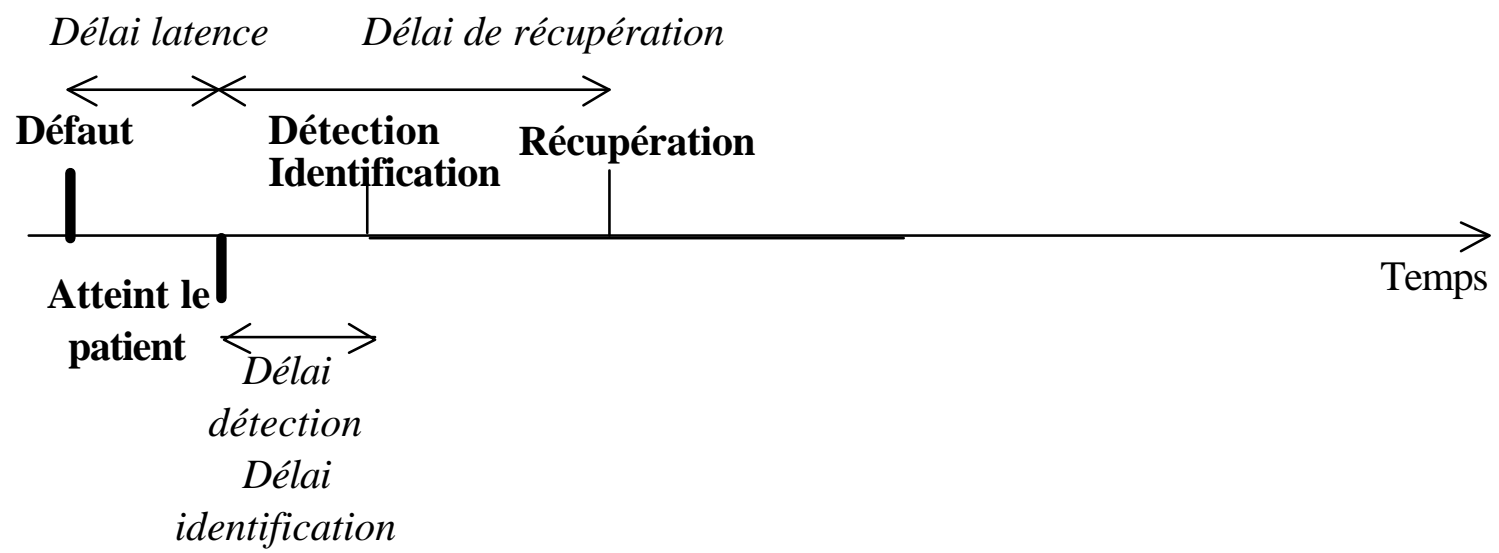

Graphique 17 : Les différents délais dans le cas où cela atteint le patient (cas 2)

\section{Situation B3}

La situation B3 représente le cas où la détection et l'identification se font au moment même où cela atteint le patient. La personne qui fait l'erreur la réalise au moment même où elle la fait. Par exemple, une infirmière administre un médicament par la mauvaise 
voie et réalise son geste aussitôt. La détection et d'identification sont donc immédiates. Cela va permettre de commencer plus rapidement la phase de récupération.

\section{Délai de récupération}

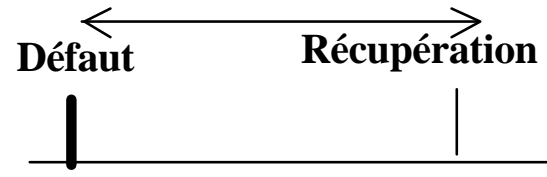

Atteint le patient

\section{Détection}

\section{Identification}

Graphique 18 : Les différents délais dans le cas où cela atteint le patient (cas 3 )

\section{Situation B4}

Dans certains cas, il se peut que des mesures soient prises avant même que la situation ait été correctement identifiée. Il s'agit souvent d'interventions pour stabiliser la situation. Ces interventions «anticipées » peuvent être propices à d'autres erreurs. Cela peut conduire à un SCÉNARIO DE CUMUL. On parle alors de cumul d'erreurs associées à la phase de récupération anticipée.

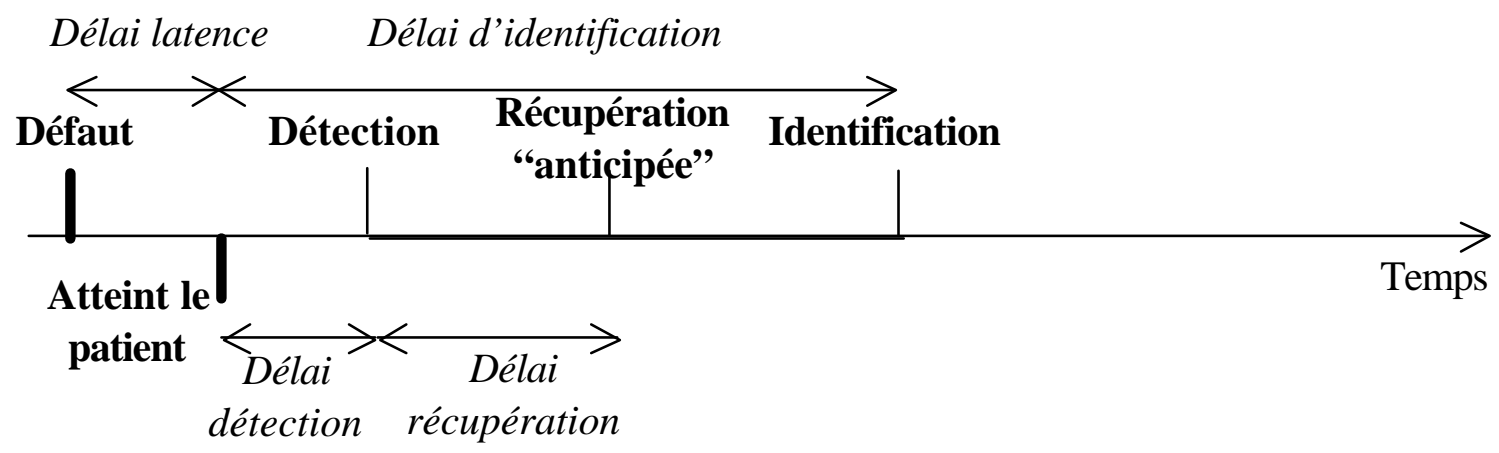




\section{Graphique 19 : Les différents délais dans le cas où cela a atteint le patient (cas 4)}

Dans le cas où cela atteint le patient, la procédure de récupération est plus complexe car elle peut faire appel de façon obligatoire à d'autres acteurs que ceux qui ont détecté ou identifié le problème. Même si l'infirmière peut poser les premiers gestes pour stabiliser la situation (début de la phase de récupération), elle devra prévenir le médecin qui fera un diagnostic et décidera des autres gestes à poser ou de l'antidote à administrer. La récupération « anticipée » peut aussi conduire à poser des gestes non adaptés à la situation réelle - qui n'a pas encore été identifiée.

- Facteurs de dépendance : Le modèle prend en compte les dépendances entre activités, entre équipes et entre acteurs (les acteurs qui ont détecté sont-ils les mêmes que ceux qui ont récupéré la situation? Même type d'acteur dans une autre équipe, autres fonctions ?). Dans notre cas, les acteurs de l'erreur, de la détection, de l'identification et de la récupération peuvent être différents.

Par exemple, une infirmière dans l'unité peut identifier une erreur faite par le pharmacien à la pharmacie et ne pas administrer le mauvais médicament au patient. Un pharmacien peut identifier une erreur de prescription du médecin et le prévenir. Dans ce cas là, le médecin modifiera sa prescription. Le médecin peut se rendre compte d'une inversion de dossiers par une résidente et ne pas procéder à des tests sur le mauvais patient. L'infirmière chef du bloc opératoire peut identifier que le matériel n'a pas été stérilisé (l'étiquette de contrôle n'est pas de la bonne couleur).

Evidemment, il y a aussi le cas où il s'agit du même acteur. On peut penser que dans ce cas là les délais sont raccourcis mais cela n'est pas aussi évident, et il y a des cas où il n'y a pas de délai : chacune des étapes étant effectuée de façon quasi-simultanée. Reprenons l'exemple de l'infirmière qui au moment même où elle fait l'injection par la mauvaise voie, elle s'en rend 
compte (situation B2). Elle a donc détecté et identifié le problème au même moment. Même si comme on l'a dit, cela devrait réduire le délai de récupération, elle ne pourra peut-être pas récupérer la situation au même moment. Toutefois, elle pourra commencer la phase de récupération aussitôt (en appelant immédiatement un médecin par exemple ou en appliquant une procédure en place, ...).

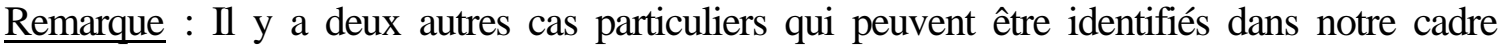
d'analyse :

1) Gestes posés pendant la phase de récupération par un autre établissement

Supposons que l'on considère un établissement en particulier, il se peut que la défaillance ait eu lieu en dehors de l'établissement et si cela a touché le patient, il se peut que le patient soit admis ou transféré pour la récupération uniquement (ou peut-être avant pour la détection si des tests sont nécessaires et qu'aucun geste n'a encore été posé).

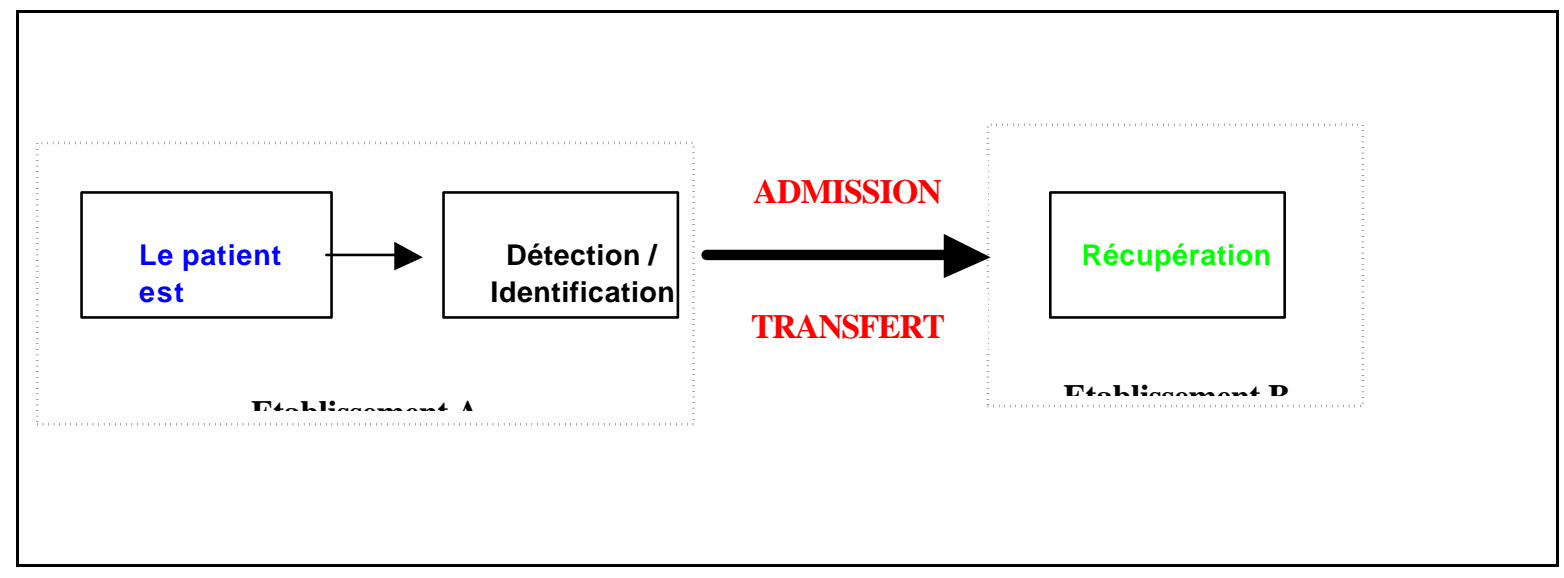

Graphique 20 : Récupération par un autre établissement

2) Sortie du patient « touché » avant détection 
L'autre cas qui peut se présenter est lorsque la défaillance a eu lieu dans un établissement et que le patient soit sorti avant même que l'on ait détecté la situation. Dans certains cas, le patient sera réadmis. Mais plus le moment de la détection est éloigné de la date de sortie du patient et plus il sera difficile d'établir le lien de causalité avec l'établissement et d'identifier les causes exactes.

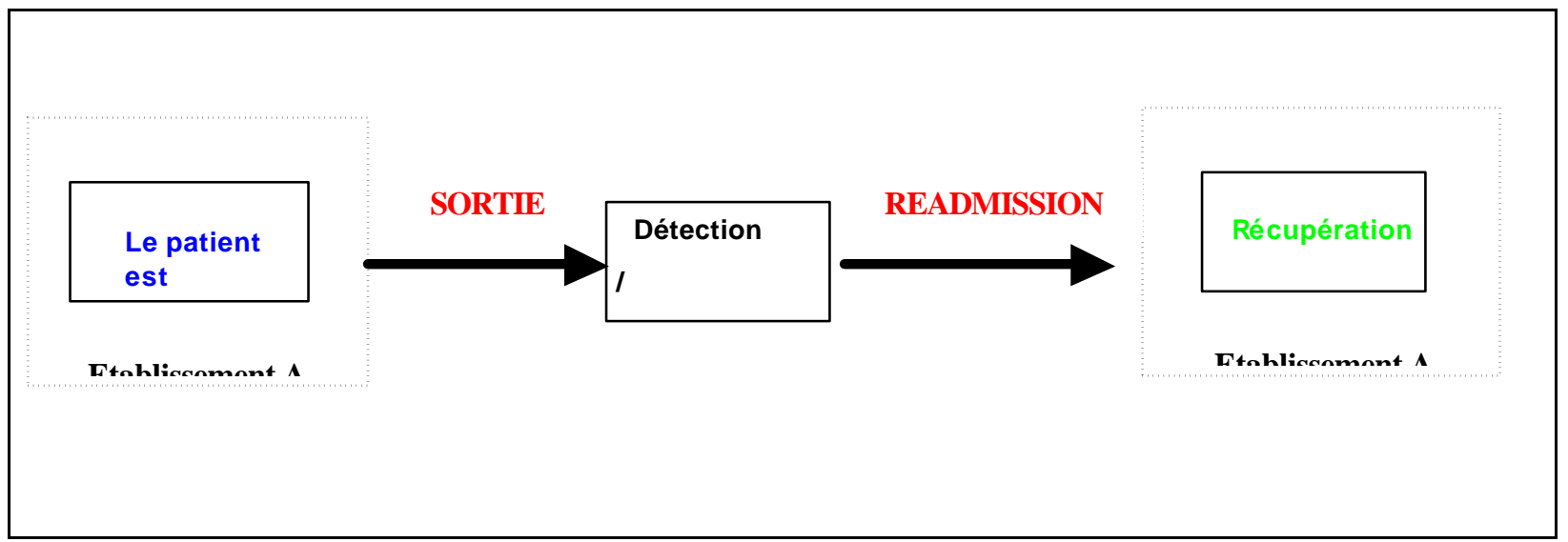

Graphique 21 : Identification après la sortie de l'usager

En effet, les conséquences de l'erreur peuvent apparaître dans les minutes qui suivent (comme dans l'industrie aéronautique), avoir un développement lent (comme dans l'industrie nucléaire) ou un développement très lent (comme cela peut être le cas pour certaines infections). Ainsi, plus le délai est important et plus il est difficile d'établir le lien de causalité. 
[Insérer graphique 22 : Déroulement de la gestion de la situation ] 


\subsection{Grille d'analyse des événements indésirables liés à la prestation des soins élaborée à partir du modèle RECUPERARE-SANTE}

En nous basant sur le modèle précédent (RECUPERARE-SANTE), nous allons proposer une grille d'analyse approfondie de certains incidents et accidents dans la prestation des soins de santé. Cette grille d'analyse est un outil qui permettra de conduire une analyse approfondie en se basant sur un certain nombre de données recueillies (données qui caractériseront l'incident ou l'accident et qui identifieront les paramètres importants). Son utilisation est donc un peu différente de celle du Nucléaire car ici seul un rapport de déclaration d'incident/accident sera rempli (avec dans certains cas un rapport complémentaire - comme cela se fait déjà dans différents établissements) mais la grille servira aussi de base à l'analyse pour certains établissements qui n'utilisent pas d'autres outils. Il est vrai que pour les autres établissements cela servira comme pour RECUPERARE-Nucleaire à standardiser le recueil des données pour les enregistrer dans une base de données.

\subsubsection{Présentation de l'outil d'analyse approfondie : la GRILLE D'ANALYSE}

Dès qu'un événement indésirable se produit, de nombreuses questions apparaissent :

Que s'est-il passé ? (description de l'événement, gravité, individu ou équipement impliqué, autre service impliqué),

Où est-ce arrivé ? (localisation / spécialité),

Quand est-ce arrivé ? (date et heure),

Comment est-ce arrivé ? (causes immédiates),

Pourquoi est-ce arrivé ? (causes sous-jacentes, facteurs de contexte, arbre des causes,...)

Quelle action a été entreprise ou proposée ? (immédiatement ou à long terme), 
Quel impact cet événement at-il eu ? (en terme de préjudice causé à l'organisation, patient, visiteur ou autres), Quels facteurs auraient pu ou ont minimisé l'impact de cet événement?

Les réponses à l'ensemble de ces questions et surtout l'analyse de ces réponses permettraient d'apprendre des erreurs commises et d'améliorer la sécurité des patients. L'outil d'analyse que nous proposons, la GRILLE, aide à répondre à ces interrogations en structurant la collecte d'information, les interviews avec les gens impliqués et en favorisant l'exploration de plusieurs pistes. Il ne s'agit pas d'une liste de vérification mais d'une analyse bâtie sur un «modèle d'incident » avec une classification liée aux facteurs clés.

\section{LA GRILLE D'ANALYSE CIRANO COMPORTE 5 SECTIONS :}

- Section $1:$ Informations générales sur l'incident/accident (AH-223)

- Section 2: Informations complémentaires sur le déroulement et les conséquences de l'incident /accident,

- Section 3 : Retour sur l'incident/accident (enquête, codification des causes et facteurs de contextes, et évaluation des performances)

- Section 4 : Mesures de prévention et recommandations

- Section 5 : Annexes.

Dans les sections 1 et 2, on retrouvera l'ensemble des informations contenues dans le rapport d'incident/accident AH-223 et aussi d'autres informations absentes de ce rapport, mais que nous avons jugé pertinentes pour l'analyse. Dans la section 2 «déroulement de l'incident /accident », nous avons essayé de mettre en évidence une phase de «récupération de 
l'incident/accident » et ainsi l'aspect gestion de l'incident par l'équipe de soins. Nous cherchons à identifier les interactions et les délais entre les différentes étapes du déroulement de l'incident. La section 3, qui est l'analyse de l'incident/accident est basée sur le retour d'expérience, prenant en compte le facteur humain, les interactions complexes entre les individus, les produits,

la technologie et les systèmes organisationnels. Cette analyse devrait permettre d'identifier : les causes (humaines, techniques et organisationnelles), les facteurs de contexte de l'événement (facteurs personnels, environnement, interface homme-machine,...) et la performance de la gestion de l'incident. L'analyse approfondie des incidents et accidents est une méthode puissante d'apprentissage de l'organisation des soins. L'analyse organisationnelle conduit directement aux stratégies d'amélioration de la sécurité du patient. Nous allons proposer dans les pages suivantes une représentation du modèle RECUPERARE-SANTE (adapté de la modélisation proposée par Baumont et al. (1999, 2000). Nous allons représenter les différentes sections du modèle : généralités, causes, récupération, performances, processus et conséquences.

\subsubsection{La GRILLE d'analyse CIRANO}

A partir du modèle RECUPERARE-SANTE, nous avons construit une grille d'analyse qui peut servir d'outil d'analyse approfondie (un guide d'accompagnement et une feuille de route pour les entretiens seront fournis avec cette grille) ou qui peut permettre le report des résultats de l'analyse approfondie pour les établissements qui utilisent déjà d'autres outils. En effet, dans un souci de standardisation minimum, il est important que le rapport d'analyse soit identique et que les informations reportées dans chaque registre local soient comparables. La GRILLE-CIRANO peut servir de support à la collecte des données pour le registre local et donc faciliter le report des données au niveau provincial. Les différentes sections de la GRILLE CIRANO vont être présentées ci-après. Le guide d'accompagnement pour l'utiliser comme outil d'analyse et la feuille de route des entretiens font l'objet d'un autre rapport. Une version informatisée est en cours de développement. 
[Insérer graphique 23 : Modèle RECUPERARE-SANTE] 
[Insérer GRILLE d'analyse CIRANO 18 pages]

PAGE 1 
[Insérer GRILLE d'analyse CIRANO 18 pages]

PAGE 2 
[Insérer GRILLE d'analyse CIRANO 18 pages]

PAGE 3 
[Insérer GRILLE d'analyse CIRANO 18 pages]

\section{PAGE 4}


[Insérer GRILLE d'analyse CIRANO 18 pages]

PAGE 5 
[Insérer GRILLE d'analyse CIRANO 18 pages]

PAGE 6 
[Insérer GRILLE d'analyse CIRANO 18 pages]

PAGE 7 
[Insérer GRILLE d'analyse CIRANO 18 pages]

PAGE 8 
[Insérer GRILLE d'analyse CIRANO 18 pages]

PAGE 9 
[Insérer GRILLE d'analyse CIRANO 18 pages]

PAGE 10 
[Insérer GRILLE d'analyse CIRANO 18 pages]

\section{PAGE 11}


[Insérer GRILLE d'analyse CIRANO 18 pages]

PAGE 12 
[Insérer GRILLE d'analyse CIRANO 18 pages]

\section{PAGE 13}


[Insérer GRILLE d'analyse CIRANO 18 pages]

PAGE 14 
[Insérer GRILLE d'analyse CIRANO 18 pages]

PAGE 15 
[Insérer GRILLE d'analyse CIRANO 18 pages]

PAGE 16 
[Insérer GRILLE d'analyse CIRANO 18 pages]

PAGE 17 
[Insérer GRILLE d'analyse CIRANO 18 pages]

PAGE 18 


\section{PROPOSITION D'UNE DÉMARCHE STRUCTURÉE D'ANALYSE APPROFONDIE DES INCIDENTS/ACCIDENTS ÉVITABLES LIÉS À LA PRESTATION DES SOINS}

L'utilisation de la grille d'analyse approfondie présentée dans la section précédente s'imbrique dans une DÉMARCHE STRUCTURÉE D'ANALYSE APPROFONDIE (notamment pour savoir dans quel cas il est recommandé ou nécessaire de faire ce type d'analyse et le suivi). Vincent et al. (2000) considèrent que l'utilisation d'un protocole en bonne et due forme permet de faire en sorte que l'enquête soit systématique, circonstanciée et rentable. Le protocole réduit le risque d'explications simplistes et de rejet systématique de la faute sur quelqu'un. Nous allons présenter la démarche et détailler chacune des étapes. Les établissements pourront utiliser notre GRILLE d'analyse CIRANO lors de l'étape de recueil d'informations complémentaires et pour mener l'analyse des causes et de la gestion de l'incident/accident ou utiliser d'autres outils qu'ils auront eux-mêmes développés et /ou qu'ils utilisent déjà. Toutefois, le rapport d'analyse (qui sera en quelque sorte le résumé de l'analyse) devra être standardisé et compilé dans la base de données de l'établissement (le registre local).

\subsection{Le déclenchement de l'analyse approfondie}

Une analyse approfondie ne peut pas être faite pour chaque incident ou accident qui surviendrait dans l'établissement. Il faut établir une liste de caractéristiques qui permettraient de « déclencher » une telle analyse. Il est important que ces critères soient le plus objectif possible pour que le déclenchement soit systématique et ne repose pas sur une procédure d'évaluation subjective. Par contre, il est important de laisser la possibilité à chacun des membres de l'organisation de solliciter une analyse approfondie pour un événement, incident ou accident qui ne correspondrait pas aux critères déclencheurs. Il peut s'agir de tout événement dont l'analyse pourrait être fructueuse en terme d'apprentissage pour l'organisation. 


\subsubsection{Les critères «déclencheurs » généralement retenus}

Dans la littérature sur les analyses approfondies d'incident ou d'accident, ce sont souvent les événements sentinelles qui servent de signal d'alerte et déclenchent systématiquement une investigation et une analyse poussée (JCAHO, 2001 ; ANAES, 2003 ). Les événements sentinelles correspondent généralement à des événements rares. Le but n'est pas une analyse statistique mais bien un retour d'expérience pour comprendre ce qui s'est passé. Ils peuvent correspondre à des événements pour lesquels les professionnels souhaitent exercer une vigilance (par exemple, le décompte en salle d'opération); ils sont donc parfois choisis par un secteur d'activité et/ou par l'établissement. Nous allons tout d'abord passer en revue les recommandations qui sont données dans différents pays et par différentes organisations concernant les cas où une analyse approfondie est recommandée. Dans certains pays, il s'agit d'événements qui peuvent faire aussi l'objet d'une surveillance nationale.

Aux Etats-Unis, la Joint Commission on Accreditation of Health Care Organizations (JCAHO) a établi une liste d'événements sentinelles ${ }^{16}$ qui peuvent leur être reporté de façon volontaire par les établissements. Ces mêmes événements devraient déclencher une analyse approfondie basée sur les principes de la «root causes analysis ». D’une manière générale, il s'agit de faits graves :

\section{Exemples d'événements sentinelles proposés par le JCAHO}

- Décès, paralysie, coma ou invalidité permanente associé à une erreur de médication ;

- $\quad$ Le suicide d'un patient;

- Toute chirurgie sur le mauvais patient, le mauvais côté ou le mauvais organe;

- Le décès d'une patiente lors de l'accouchement;

- Le décès d'un nouveau-né non relié à un problème congénital ;

- Tout homicide;

- Une chute d'un patient qui entraîne son décès ou une invalidité permanente ;

- Un accident transfusionnel impliquant une incompatibilité de groupe sanguin.

\footnotetext{
${ }^{16}$ Cette liste est disponible sur le site de la JCAHO : www.jcaho.org
} 
En Angleterre, l'évaluation de la gravité des incidents se fait à l'aide d'une grille encore en phase d'évaluation. Les incidents graves doivent être déclarés à l'Agence nationale de sécurité des patients créée fin 2001 et faire l'objet d'une analyse approfondie par l'établissement.

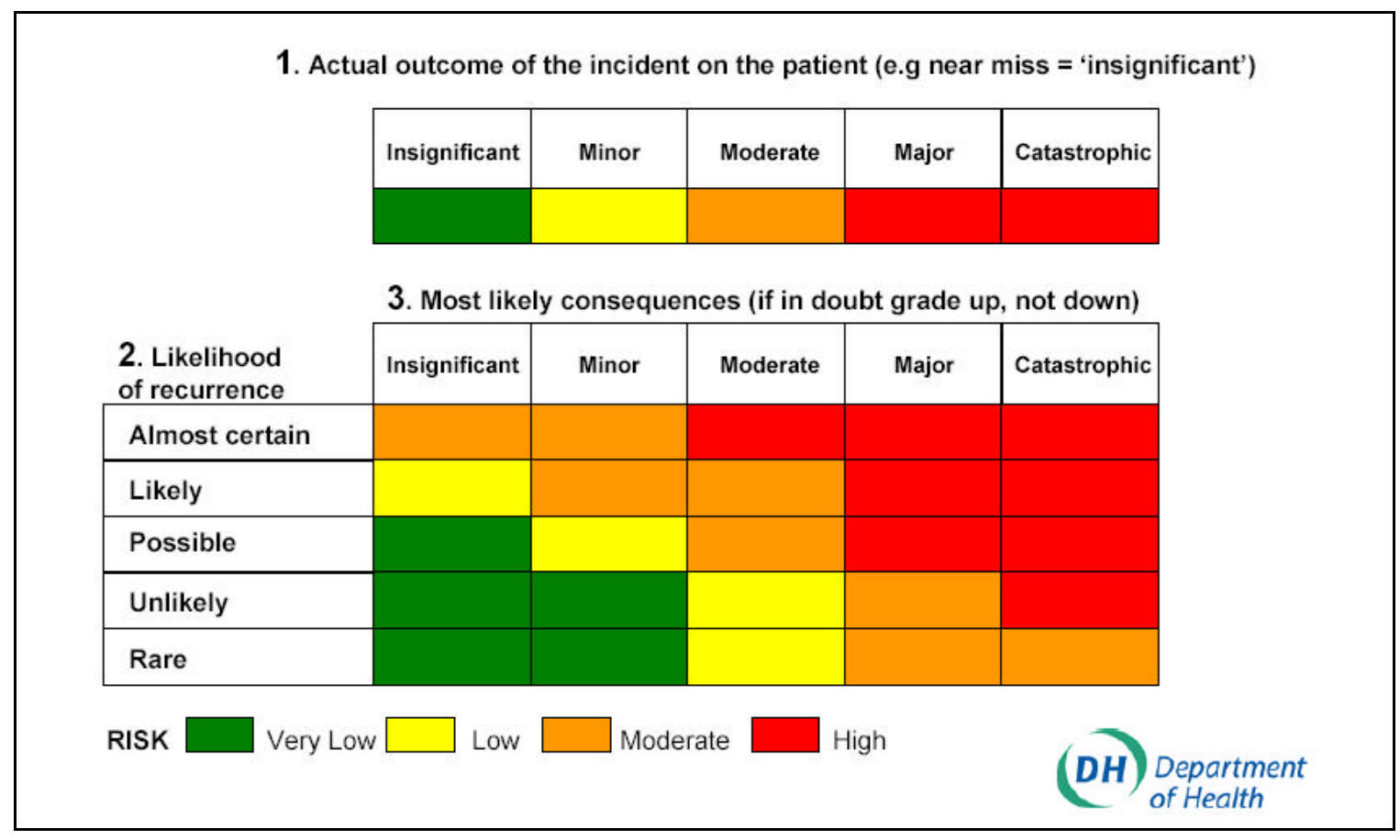

Graphique 24 : Grille d'évaluation de la gravité des incidents utilisée en Angleterre (NHS, 2001)

En France, l'Agence Nationale d'Accréditation et d'Evaluation en Santé donne un certain nombre d'événements qui pourraient faire l'objet d'une analyse approfondie (mais il n'y a aucune obligation)

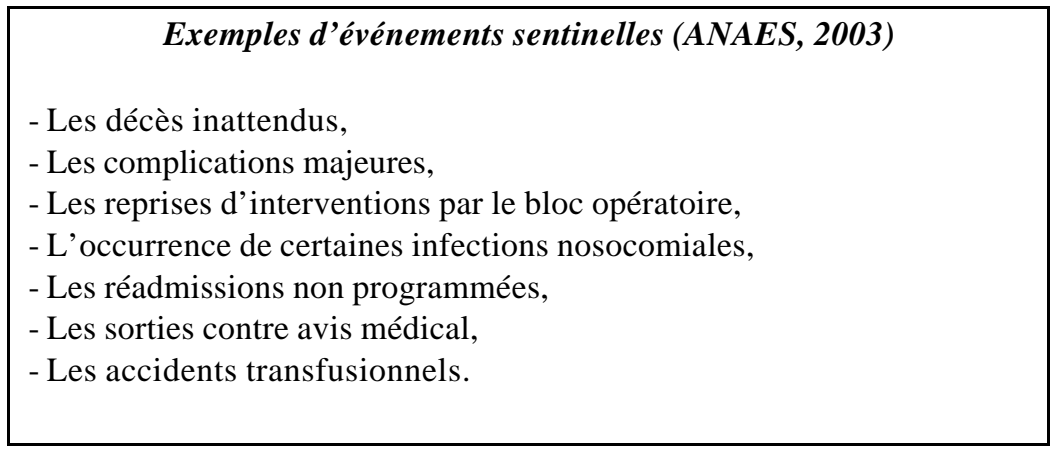


Pour les Hôpitaux Universitaires de Genève (Suisse), les incidents qui entrent dans la catégorie « faits graves » doivent être annoncés par les professionnels de soins au secrétariat général et aux directeurs médical et infirmier, et cela même s'ils ont été déclarés à un groupe de gestion des incidents au niveau du service (Choppard \& Wasserfallen, 2002).

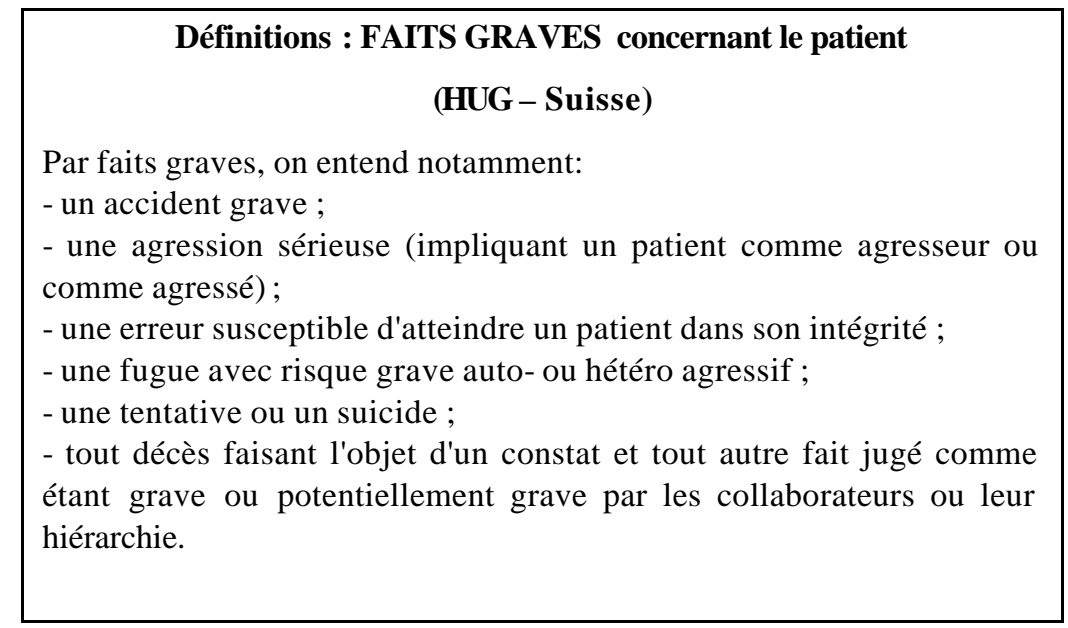

Dans les hôpitaux québécois que nous avons rencontrés, les critères qui déclenchent une analyse approfondie sont plus ou moins bien déterminés et les procédures ne sont pas toujours systématiques. Certains hôpitaux ont défini des critères indiquant dans quels cas la direction des soins infirmiers ou le gestionnaire de risque doivent être avisés ${ }^{17}$ (c'est d'ailleurs souvent la DSI qui contacte dans un second temps le gestionnaire de risques). Il s'agit la plupart du temps de cas graves et d'événements sentinelles. Les cas ou une poursuite est prévisible peuvent être aussi des déclencheurs, l'enquête servant à documenter le dossier de litige.

Exemple 1: Dans l'un des établissements visités, les types d'événements à risque devant être rapportés à la gestionnaire de risques sont clairement identifiés. Les critères dépendent soit du type d'événement, soit de la conséquence.

\footnotetext{
${ }^{17}$ Il s'agit la plupart du temps de contacts téléphoniques.
} 


\begin{tabular}{|l|}
\hline \multicolumn{1}{c|}{$\begin{array}{c}\text { Exemples d'événements devant être rapportés } \\
\text { a la gestionnaire de risque }\end{array}$} \\
Toute adminis tration d'une dose supérieure à la dose thérapeutique (ex. \\
10 fois la dose de médicament prescrit) \\
- Tout fonctionnement douteux d'un équipement qui entraîne son retrait \\
des unités de soins \\
- Toute aiguille manquante lors du décompte final au bloc opératoire \\
- Toute chute entraînant une fracture \\
- Toute fugue et tentative de suicide
\end{tabular}

Exemple 2: Un autre établissement s'est inspiré de l'échelle de NCC MERP utilisée pour les erreurs de médication (voir Annexe 2) et l'a modifiée pour l'adapter à tous les types d'événements. La classification va de la cote $A$, pour les circonstances ou événements qui auraient pu entraîner une erreur à la cote $I$, où il y a eu erreur entraînant la mort de l'usager. Les utilisateurs du AH-223 inscrivent la cote à un endroit donné sur le rapport. Les rapports ayant une cote de F à I sont automatiquement transmis au gestionnaire de risques.

\subsubsection{Les critères qui pourraient être retenus}

Les hôpitaux doivent définir clairement les critères déclencheurs et les communiquer à l'ensemble des membres de l'établissement. Dans le but de construire une banque de données national e, ces critères devraient être communs à tous les établissements. Dans notre cadre conceptuel, le déclenchement d'une analyse approfondie pourrait être prévu lors du signalement soit : d'un CRITÈRE DE GRAVITÉ, d'un ÉVÉNEMENT PRÉDÉTERMINÉ (sentinelle), ou lorsqu'un ÉVÉNEMENT EST FRÉQUENT (critères de fréquence) ou pour TOUT ÉVÉNEMENT DONT L'ANALYSE POURRAIT ÊTRE FRUCTUEUSE en terme d'apprentissage pour l'organisation. 


\section{- Critères de gravité}

Tous les événements ayant entraîné l'une (ou plusieurs) des conséquences ci-dessous :

Décès ;

Dommage sévère et/ou permanent (Exemple : paralysie, chirurgie sur le mauvais patient ou le mauvais membre, fracture, brûlure deuxième et troisième degré, désordre physiologique important, etc.);

$>$ Tout événement qui prolonge ou entraîne l'hospitalisation, si identifié ;

$>$ Transfert aux soins intensifs ;

$>$ Quasi-décès (choc anaphylactique, réanimation) .

\section{- Liste d'événements prédéterminés (événements sentinelles)}

D'autres événements devraient être analysés sans regard aux conséquences parce que leur incidence en soi est grave :

> Anormalité dans le décompte (aiguilles, compresses, etc.);

$>$ Équipements et matériels non stériles disponibles à l'utilisation (utilisés ou non) ;

D Autres critères reliés à des services en particulier ( Par exemple : bris de confidentialité ou perte de document aux archives) ;

Certains événements reliés à la médication impliquant des classes particulières de médicament (par exemple : les familles d'antinéoplasique, et d'hyperglycémiant, etc. ).

\section{- Critères de fréquence}

Les critères de fréquence ne peuvent pas être utilisés de la même façon que les critères de gravité ou que la liste d'événements prédéterminés. Actuellement, tous les établissements ne compilent pas leurs rapports, et ceux qui le font ne le font pas nécessairement dans des délais qui permettent d'inclure une alarme dans le système pour avertir lorsque plusieurs événements similaires se produisent. Par exemple, si 5 rapports d'incident/accident étaient complétés la même semaine ou le même mois en ayant le même objet et sous-objet, cela devrait alarmer l'établissement. L'analyse approfondie peut être demandée par le chef du service ou par la gestionnaire de risque, qui peut avoir eu connaissance d'un nombre élevé 
d'événements du même type. Les critères de fréquence tiendront compte des conséquences ou des conséquences potentielles. Peu de cas sont nécessaires pour déclencher une analyse approfondie si les conséquences potentielles sont graves. Par exemple, une infirmière de l'unité de néonatologie reçoit le mauvais médicament de la pharmacie. Cet événement s'est produit 3 fois au cours de la dernière semaine. Aucun accident n'a eu lieu parce que les mécanismes de vérification ont bien fonctionné. Par contre, les conséquences potentielles et la fréquence dans ce cas exigent une analyse approfondie pour identifier les causes.

\section{- Tout autre événement dont l'analyse peut être fructueuse en terme d'apprentissage pour l'organisation.}

Les critères déterminés ne doivent pas être exclusifs. Une analyse approfondie peut être effectuée pour tout autre événement dont l'analyse peut être fructueuse en terme d'apprentissage pour l'organisation. Dans ce dernier cas, il peut s'agir de demandes faites expressément par les différents comités de l'établissement, comme le comité de Mortalité \& Morbidité de l'établissement ou à partir d'informations disponibles dans l'ensemble de la vie de l'établissement (ANAES, 2003). Par exemple, il peut s'agir de risques identifiés par les professionnels et communiqués à l'encadrement, ou de risques identifiés par les différentes instances de l'établissement (DSI, DSP, CMDP, comité de sécurité transfusionnelle, comité de santé et sécurité au travail, cellule d'hygiène,...). Cela pourrait aussi être déclenché par des plaintes ou une réclamation exprimée par les patients ou leurs familles mais aussi pour des risques identifiés par la démarche qualité ou l'accréditation ou lors d'audits organisationnels. Il peut s'agir de problème de coordination entre les différents services.

«Idéalement », tous les services (services de soins, entretien, laboratoire, radiologie, etc.) devraient utiliser la même structure de remontée d'informations et les mêmes critères déclencheurs (voir le graphique ci-dessous). 


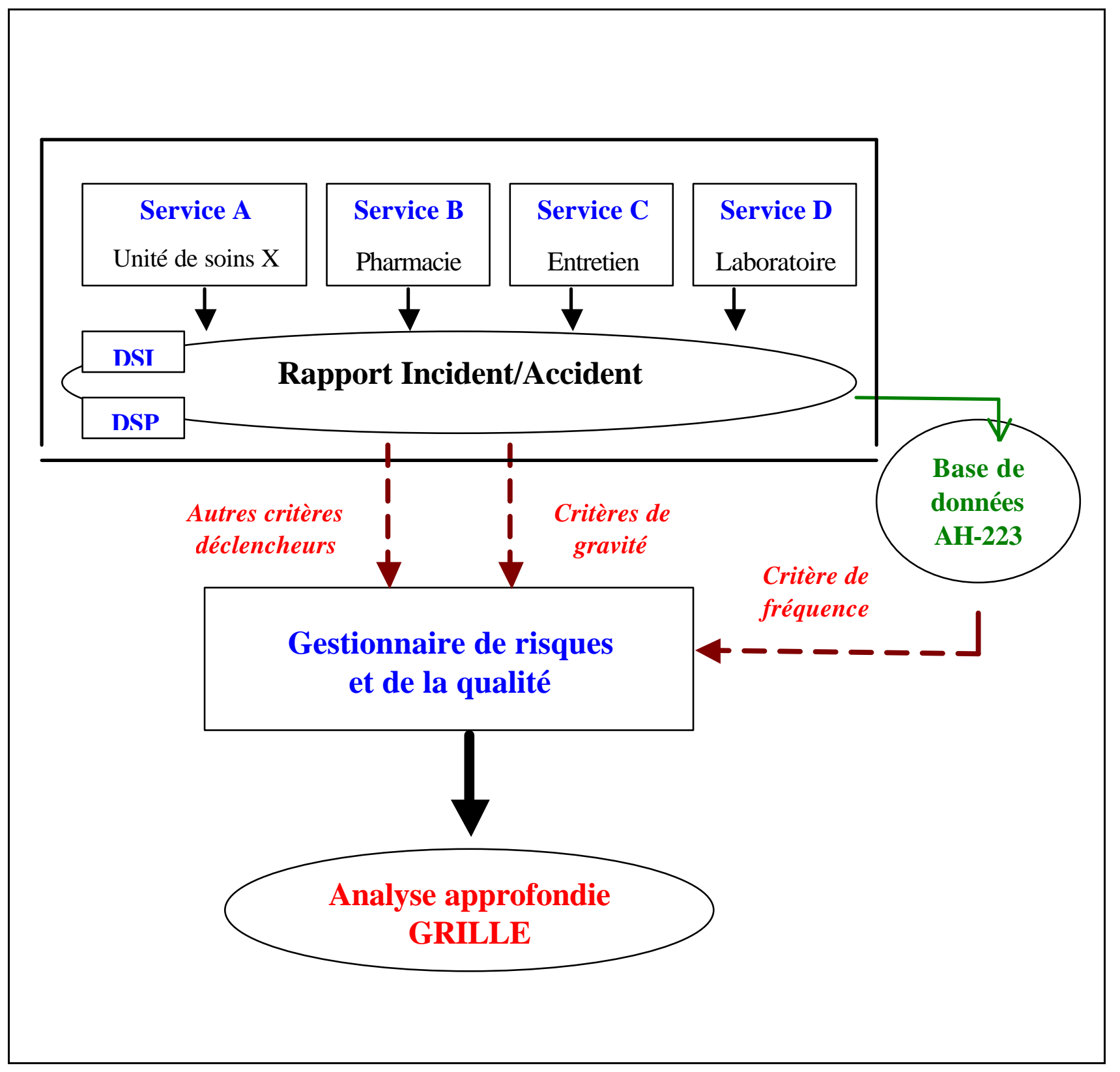

Graphique 25 : La gestion des rapports d'incident/accident et le déclenchement de l'analyse approfondie 


\subsection{Les étapes de la démarche d'analyse approfondie}

Le modèle nécessite que l'analyste commence par examiner la succession d'événements ayant conduit à un accident ou à une issue défavorable et par considérer les actes des personnes impliquées. Ensuite, il est crucial que l'analyste remonte jusqu'aux conditions dans lesquelles le personnel travaillait et le contexte organisationnel dans lequel l'incident/accident est survenu. L'utilisation d'une démarche structurée garantit une analyse systématique, exhaustive et efficace. Toute analyse approfondie doit consister en une série d'étapes à suivre :

\section{Etape 1: S'assurer que l'incident ou l'accident déclaré nécessite bien une analyse approfondie et commencer le processus d'analyse approfondie}

Suite à un événement indésirable répondant à des critères péalablement établis dont nous avons discuté ci-dessus, la personne en charge de la lecture des rapports, habituellement l'infirmière chef, la DSI ou la gestionnaire de risque, va déclencher le processus d'analyse approfondie (s'il y a le moindre doute, une vérification préalable avec d'autres personnes de l'équipe ou la gestionnaire de risque est recommandée). L'analyste (qui est dans la plupart des cas la gestionnaire de risque ou l'une des personnes de son équipe désignée pour faire les analyses) est contacté et le contact se fait initialement par téléphone dans les heures suivant l'événement s'il s'agit d'un événement grave ou listé. Pour les autres types d'événements (identifiés par le critère de fréquence ou tout autre événement pour lequel on souhaite qu'une analyse approfondie soit faite), il suffit de contacter l'analyste pour lui exposer la pertinence du cas proposé.

\section{Etape 2: L'analyste se rend sur les lieux de l'événement et rencontre la personne qui a complété le rapport d'incident/accident}

L'analyste se rend sur les lieux de l'incident/accident. Il observe et documente les lieux

physiques. Il revoit le rapport d'incident/accident avec la personne qui l'a complété en y ajoutant les informations qui ne s'y trouvent pas. Il établit ainsi quand l'événement est survenu, qui en a été victime (s'il y a lieu), et qu'est-il arrivé, c'est à dire, la nature de 
l'événement, la description des faits, les informations sur la situation avant et au moment de l'événement, comment a-t-on détecté et identifié l'événement, quelles actions ont été entreprises pour récupérer la situation et éviter qu'elle ne se reproduise, la séquence chronologique des événements et quelles sont les conséquences de l'événement.

Si la GRILLE d'analyse CIRANO est utilisée, l'analyste remplit les sections 1 et 2. Le but de cette première étape est d'établir les circonstances telles qu'elles apparaissent au premier abord et préparer une feuille de route (documenter un résumé initial, préparer une première chronologie des événements, identifier tout problème évident lié aux soins). À ce point, l'analyste :

Identifie et organise des rencontres avec les autres personnes directement impliquées ou présentes lors de l'incident/accident ;

S'assure que des mesures correctrices pour empêcher la récurrence de ce même incident/accident, sont mises en place immédiatement.

\section{Etape 3: L'analyste rencontre les gens impliqués ou les témoins (incluant le patient si nécessaire)}

Dans les heures ou jours suivant l'événement (dans un délai raisonnable), l'analyste rencontre les gens impliqués, autres que la personne ayant complété le rapport. Ces rencontres se font sur la base d'interviews semi-dirigées, c'est à dire que l'analyste cherche à obtenir des informations qui seront pertinentes pour l'analyse et pose une série de questions. Il cherche à :

Etablir la chronologie des événements ;

Passer en revue la séquence des événements et poser des questions sur chaque problème en identifiant l'étape de la séquence ; 
Identifier les causes et les facteurs de contexte de chaque problème (si la grille CIRANO est utilisée, l'analyste pourra se servir de l'aide (feuille de rappel) pour poser des questions supplémentaires)

Reconstruire la phase de gestion de la situation une fois que le patient a été touché.

Identifier ce qui aurait pu être fait pour que l'incident ou l'accident n'ait pas lieu.

Identifier les mesures pouvant être prises pour éviter qu'un tel événement se reproduise.

Ces rencontres doivent se faire sur une base individuelle. Il est cependant possible que dans certains cas des rencontres se fassent en groupe (notamment dans les cas où plusieurs services sont impliqués) mais cela aura souvent lieu dans un second temps, au moment du retour sur l'accident pour prendre des mesures et mettre en place des mesures de prévention adaptées. Le nom des personnes rencontrées, leur fonction, l'heure et la date de la rencontre doivent bien être indiqués. De plus, un compte-rendu des rencontres doit être conservé et mis en annexe de l'analyse.

Normalement, à ce point l'analyste a toutes les informations pour reconstruire la séquence des événements. Il est cependant possible que l'analyste découvre des pistes nouvelles à explorer lors des interviews et qu'il doive rencontrer d'autres acteurs non prévus initialement. L'analyste cherchera à découvrir la cause racine. Par exemple, dans le cas où un usager recevrait une surdose de médicament, l'infirmière qui a complété le rapport AH-223 dit avoir donné le médicament tel qu'il était indiqué sur l'étiquette. L'analyste doit maintenant déterminer si c'est la pharmacie ou l'ordonnance qui est en cause.

\section{Etape 4 : L'analyste collige et rassemble les informations}

Une fois les rencontres terminées, l'analyste doit colliger et rassembler les différents éléments d'analyse. Dans un premier temps, il doit déterminer la séquence des 
événements et identifier à la fois le contexte général, la charge de travail des personnes impliquées, les causes et les facteurs contributifs associés à chacun des événements. La section 3 de la GRILLE CIRANO propose une liste non exhaustive des causes et des facteurs de contexte. Une fois l'information colligée, l'analyste doit être en mesure de RECONSTRUIRE L'ARBRE DES CAUSES. Il devra aussi colliger l'ensemble des informations concernant la gestion de la situation.

\section{Etape 5: Mise en place de mesures correctrices et recommandations}

L'analyste reprend l'ensemble des informations concernant ce qui aurait pu être fait pour que l'incident ou l'accident n'ait pas lieu et les mesures proposées pouvant être prises pour éviter qu'un tel événement se reproduise pour faire ses recommandations auprès des personnes concernées. De plus, les points positifs et négatifs de la gestion de l'accident doivent être identifiés dans le but d'améliorer la performance lors

d'événements similaires. Étant donné qu'il est impossible d'éliminer complètement l'occurrence des événements indésirables, il est important d'apprendre des événements passés pour mettre en place des mécanismes pour réduire les conséquences des événements futurs.

Dans certains cas, une réunion des chefs de services et représentants des professions impliquées est nécessaire pour évaluer les diverses possibilités de mesures correctives qui pourraient être mises en place et pour susciter des commentaires et suggestions informées.

Ces recommandations doivent aussi être codifiées. L'analyste pourra utiliser la section 4 de la GRILLE à cette fin. Par exemple, il peut être notifié que la personne en cause dans l'événement reçoive une formation appropriée ou que la procédure associée au transfert d'un usager d'un établissement à un autre soit révisée ou mise à jour.

\section{Etape 6 : Rédaction du rapport d'analyse}


Une fois l'analyse complétée, l'analyste doit rédiger un rapport d'analyse. Ce rapport, assez court et dénominalisé, doit décrire brièvement les faits et dresser la liste des causes, des facteurs de contexte, les gestes posés et les recommandations pour prévenir la répétition d'un tel événement. Ces rapports seront mis dans la base de données locale.

\section{Etape 7 : Retour auprès des personnes impliquées}

L'analyste fait part de ses conclusions à tous les professionnels qu'il a rencontrés au cours de son analyse.

\section{Etape 8 : Suivi des recommandations et mesures correctrices}

Après un certain temps, l'analyste fait le suivi des recommandations et des mesures correctrices. Il s'assure que les mesures correctives ont bien été mises en place.

Etape 9: Présentation au comité de gestion des risques et de la qualité de l'établissement ${ }^{18}$

L'analyste doit toujours chercher à avoir une vue d'ensemble de la situation concernant les risques et la qualité dans l'établissement. Il présente au comité de gestion des risques et de la qualité de l'établissement plusieurs fois par année (selon la politique de l'établissement) les statistiques descriptives (type d'événements, services impliqués, conséquences,...) des incidents /accidents à partir des données recueillies sur l'ensemble des rapport d'incident/accident mais aussi concernant les événements qui ont fait l'objet d'une enquête approfondie. Il présente une analyse des analyses (performance de gestion des incidents/accidents, délais, corrélation type d'événement \& clientèle,...), les causes et les facteurs contributifs significatifs et un état des suivis. L'analyste doit aussi faire un suivi global des recommandations et des mesures correctrices. C'est à dire,

\footnotetext{
${ }^{18}$ Voir description du comité en annexe (description de la loi 113)
} 
vérifier si elles ont été adoptées. Il est possible que certaines mesures s'avèrent insuffisantes ou que certaines personnes refusent de les appliquer. L'analyste pourra en faire part au comité de gestion des risques et de la qualité de l'établissement qui pourra s'il le juge nécessaire prendre des mesures.

\section{LES ÉTAPES DE LA DÉMARCHE D'ANALYSE APPROFONDIE DES ÉVÉNEMENTS INDÉSIRABLES}

1 : S'assurer que l'incident ou l'accident déclaré nécessite bien une analyse approfondie et commencer le processus d'analyse approfondie

2 : L'analyste se rend sur les lieux de l'événement et rencontre la personne qui a complété le rapport d'incident/accident

3 : L'analyste rencontre les gens impliqués ou les témoins (incluant le patient si nécessaire)

4 : L'analyste collige et rassemble les informations

5 : Mise en place de mesures correctrices et recommandations

6 : Rédaction du rapport d'analyse

7 : Retour auprès des personnes impliquées

8 : Suivi des recommandations et mesures correctrices

9 : Présentation au comité de gestion des risques et de la qualité de l'établissement 


\section{CONCLUSION}

Le changement conceptuel amorcé dans les systèmes de soins autour de la gestion des incidents et des accidents en est encore à ses débuts. La loi 113 a accéléré les réflexions et la mise en place d'une structure dédiée à la gestion des risques et à la qualité dans les établissements au Québec. L'adhésion de chaque professionnel de la santé est un pré-requis indispensable à la mise en place d'une politique de gestion des risques et de la qualité qui soit crédible et efficace.

Dans ce rapport, nous avons défini les bases d'un cadre normatif servant à évaluer et à colliger les divers incidents et accidents liés à la prestation des soins à l'hôpital et nous avons proposé une démarche d'analyse approfondie et un outil d'analyse des défaillances systémiques. Notre objectif était d'une part, d'aider à structurer l'analyse interne des risques d'accidents et d'incidents en milieu hospitalier tout en gardant une flexibilité pour les établissements qui utilisent déjà d'autres outils. Nous avons consulté de nombreux professionnels et différents établissements pour prendre en compte leurs besoins spécifiques. Il est important de faciliter la déclaration, de la rendre facile d'utilisation et surtout d'impliquer tous les services et tous les intervenants dans l'établissement. D'autre part, nos travaux de recherche devraient permettre une standardisation de l'analyse des causes, des facteurs de contexte et de la gestion de la situation pour permettre une remontée de certaines informations au niveau local puis nationale. Même si la base de donnée nationale sera mise en place dans un second temps (car cela demande que les registres locaux soient en place et informatisés), il ne faut pas perdre de vue cet objectif pour pouvoir proposer les mesures qui permettront de la constituer.

Ainsi, les établissements pourront utiliser notre GRILLE d'analyse CIRANO lors de l'étape de recueil d'informations complémentaires et pour mener l'analyse des causes et de la gestion de l'incident/accident ou utiliser d'autres outils qu'ils auront eux-mêmes développés et /ou qu'ils utilisent déjà. Dans le cas où d'autres outils d'analyse seraient utilisés, la GRILLE d'analyse CIRANO pourrait être utilisée comme rapport d'analyse pour reporter l'ensemble des résultats 
de l'analyse. Ces rapports seront compilés dans le registre local de l'établissement. La déclaration et l'analyse approfondie sont à utiliser dans la perspective d'un apprentissage organisationnel et doivent s'insérer dans un processus continu d'amélioration de la qualité et contribuer au changement de culture. Le recueil, l'analyse des informations recueillies par les acteurs de l'établissement sont des opportunités pour renforcer les liens de confiance entre les personnes, valoriser leurs connaissances et mieux connaître le système. Dans leurs différents volets, les travaux de recherche du CIRANO devraient aider les établissements du réseau de la santé du Québec

à se conformer aux dispositions de la nouvelle loi et aider le ministère à organiser le recueil d'informations sur les incidents et accidents survenus dans les établissements au niveau national.

La démarche structurée et la grille d'analyse approfondie que nous proposons vont être testées lors d'une étude pilote auprès d'un échantillon représentatif de centres hospitaliers du Québec. Nous souhaitons évaluer la pertinence et la faisabilité de nos outils et mesurer les «coûts » de mise en application et d'utilisation. Nous allons ensuite les adapter aux autres types d'établissements du réseau (CLSC-CHLSD,...) et proposer les bases du recueil national. En effet, même si l'objectif premier de la nouvelle loi est de permettre de savoir et comprendre ce qui se passe au sein de l'établissement, il est aussi important de savoir ce qui se passe ou ce qui aurait pu se passer d'une manière générale dans les autres établissements de la province. Pour cela il faut qu'une collecte des données soit organisée au sein de chaque hôpital, que les informations recueillies soient analysées et remontent à un niveau supérieur. La loi 113 spécifie que «le directeur général de l'établissement doit rapporter, sous forme non nominative, à la régie régionale, selon une fréquence convenue ou lorsque celle-ci le requiert, les incidents ou accidents déclarés». Cela permettrait aux établissements d'apprendre des autres expériences. De plus, un événement grave "unique" au sein de l'établissement peut ressortir au niveau national pour sa fréquence (identifié "une fois" dans plusieurs établissements). Cela peut permettre de faire prendre conscience aux autres établissements de l'importance du problème et de mettre en place les mesures de prévention adaptées. 
Les détails sur le report national seront analysés dans un autre rapport. Toutefois, il est important de bien prendre en compte cet objectif (même si cela se fera dans un second temps) car il va orienter le report local. La gestion des risques et de la qualité dans les centres hospitaliers doit se baser sur les 4 principes suivants :

\section{(1) identifier les situations à risque}

=> DECLARER tous incidents et les accidents (qui surviennent dans tous les services) à l'aide du formulaire prévu à cet effet. L'ensemble des formulaires seront colligés dans une base de données locale.

\section{(2) comprendre ce qui s'est passé}

=> ANALYSER la situation, chercher à identifier les causes et les facteurs de contexte et comment la situation a été gérée. Une analyse approfondie sera faite pour certains incidents et accidents.

\section{(3) éviter que cela ne se reproduise}

=> PREVENIR les situations à risque

\section{(4) partager les « expériences »}

=> une étude statistique de la base de données des rapports d'incidents/accidents doit être faite et les résultats doivent être transmis à l'ensemble des intervenants de l'hôpital dans tous les services. Une «analyse » des analyses approfondies doit servir à tirer des leçons des expériences passées. De plus, la transmission des informations dénominalisées doit permettre le partage des informations au niveau national .

Le graphique ci-dessous résume la démarche globale de gestion des risques et de la qualité dans les établissements de soins au Québec : 
[Insérer graphique 26 : Registre local et registre national] 


\section{ANNEXE 1 : EXTRAITS DE LA LOI 113}

\section{Projet de loi113}

(2002, Chapitre 71)

\section{LOI MODIFIANT LA LOI SUR LES SERVICES DE SANTE ET LES SERVICES SOCIAUX CONCERNANT LA PRESTATION SECURITAIRE DE SERVICES DE SANTE ET DE SERVICES SOCIAUX}

$\ldots / \ldots$

4. L'article 8 de cette loi est modifié par l'addition, à la fin, des alinéas suivants :

«Il a également le droit d'être informé, le plus tôt possible, de tout accident survenu au cours de la prestation de services qu'il a reçus et susceptible d'entraîner ou ayant entraîné des conséquences sur son état de santé ou son bien-être ainsi que des mesures prises pour contrer, le cas échéant, de telles conséquences ou pour prévenir la récurrence d'un tel accident.

Pour l'application du présent article et des articles 183.2, 233.1, 235.1 et 431 et à moins que le contexte ne s'y oppose, on entend par :

« accident » : action ou situation où le risque se réalise et est, ou pourrait être, à l'origine de conséquences sur l'état de santé ou le bien-être de l'usager, du personnel, d'un professionnel concerné ou d'un tiers. ».

$\ldots / \ldots$

9. Cette loi est modifiée par l'insertion, après l'article 183, des suivants :

«183.1. Le plan d'organisation de tout établissement doit aussi prévoir la formation d'un comité de gestion des risques et de la qualité.

Le nombre de membres de ce comité ainsi que ses règles de fonctionnement sont déterminés par règlement du conseil d'administration de l'établissement. La composition de ce comité doit assurer une représentativité équilibrée des employés de l'établissement, des usagers, des personnes qui exercent leur profession dans un centre exploité par l'établissement de même que, s'il y a lieu, des personnes qui, en vertu d'un contrat de services, dispensent pour le compte de l'établissement des services aux usagers de ce dernier. Le directeur général ou la personne qu'il désigne est membre d'office de ce comité.

«183.2. Ce comité a notamment pour fonctions de rechercher, de développer et de promouvoir des moyens visant à :

$1^{\circ}$ identifier et analyser les risques d'incident ou d'accident en vue d'assurer la sécurité des usagers ;

$2^{\circ} \mathrm{s}$ 'assurer qu'un soutien soit apporté à la victime et à ses proches ;

$3^{\circ}$ assurer la mise en place d'un système de surveillance incluant la constitution d'un registre local des incidents et des accidents pour fins d'analyse des causes des incidents et accidents et recommander au conseil d'administration de l'établissement la prise de mesures visant à prévenir la récurrence de ces incidents et accidents ainsi que la prise de mesures de contrôle, s'il y a lieu.

Pour les fins d'application du présent article et des articles 233.1, 235.1 et 
431 et à moins que le contexte ne s'y oppose, on entend par :

« incident » : une action ou une situation qui n'entraîne pas de conséquence

sur l'état de santé ou le bien-être d'un usager, du personnel, d'un professionnel

concerné ou d'un tiers mais dont le résultat est inhabituel et qui, en d'autres occasions, pourrait entraîner des conséquences.

«183.3. Les réponses faites par une personne, dans le cadre des activités de gestion des risques, et notamment tout renseignement ou document fourni de bonne foi par elle en réponse à une demande d'un gestionnaire de risques et de la qualité ou d'un comité de gestion des risques et de la qualité ne peuvent être utilisées ni ne sont recevables à titre de preuve contre cette personne ou contre toute autre personne devant une instance judiciaire ou une personne ou un organisme exerçant des fonctions juridictionnelles. Malgré toute disposition contraire, un gestionnaire de risques et de la qualité ou un membre d'un comité de gestion des risques et de la qualité ne peut être contraint devant une instance judiciaire ou une personne ou un organisme exerçant des fonctions juridictionnelles de faire une déposition portant sur un renseignement confidentiel qu'il a obtenu dans l'exercice de ses fonctions, ni de produire un document contenant un tel renseignement, si ce n'est aux fins du contrôle de sa confidentialité.

Aucun élément de contenu du dossier de gestion des ris ques et de la qualité, y compris les conclusions motivées et, le cas échéant, les recommandations qui les accompagnent, ne peut constituer une déclaration, une reconnaissance ou un aveu extrajudiciaire d'une faute professionnelle, administrative ou autre de nature à engager la responsabilité civile d'une partie devant une instance judiciaire.

«183.4. Malgré la Loi sur l'accès aux documents des organismes publics et sur la protection des renseignements personnels (chapitre A-2.1), les dossiers et les procès-verbaux du comité de gestion des risques et de la qualité sont confidentiels. Nul ne peut prendre connaissance des procès-verbaux du comité de gestion des risques et de la qualité sauf les membres de ce comité, les représentants d'organismes d'accréditation dans l'exercice des fonctions relatives à l'agrément des services de santé et des services sociaux des établissements ou les représentants d'un ordre professionnel dans l'exercice des fonctions qui leur sont attribuées par la loi. ».

10. Cette loi est modifiée par l'insertion, après l'article 233, du suivant :

«233.1. Tout employé d'un établissement, toute personne qui exerce sa profession dans un centre exploité par l'établissement, tout stagiaire qui effectue un stage dans un tel centre de même que toute personne qui, en vertu d'un contrat de services, dispense pour le compte de l'établissement des services aux usagers de ce dernier doit déclarer, au directeur général d'un établissement ou, à défaut, à une personne qu'il désigne, tout incident ou accident qu'il a constaté, le plus tôt possible après cette constatation. Une telle déclaration doit être faite au moyen du formulaire prévu à cet effet, lequel est versé au dossier de l'usager. Le directeur général de l'établissement ou, à défaut, la personne qu'il désigne rapporte, sous forme non nominative, à la régie régionale, selon une fréquence convenue ou lorsque celle-ci le requiert, les incidents ou accidents déclarés.» 


\title{
ANNEXE 2 : Classification des erreurs de médication du NCC MERP (National Coordinating Council for Medication Error Reporting and Prevention)
}

\author{
Source : NCC MERP News release no.96C04, September 4, 1996.
}

Type d'erreur

Aucune erreur

Catégorie A Circonstances ou événements qui auraient pu causer une erreur

\section{Erreur, aucun dommage}

Catégorie B

Catégorie $\mathrm{C}$

Catégorie D

Résultat (conséquence) patient. sans dommage
Une erreur est survenue mais le médicament n'a été administré au

Une erreur est survenue, le médicament a été administré au patient mais

Une erreur est survenue, le médicament a été administré au patient, nécessitant un traitement ou une intervention et a causé un dommage temporaire au patient.

\section{Erreur, dommage}

Catégorie $\mathrm{E}$

Une erreur est survenue, le médicament a été administré au patient, nécessitant un traitement ou une intervention et a causé un dommage temporaire au patient.

Catégorie F Une erreur est survenue résultant dans une hospitalisation initiale ou prolongée et a causé un dommage temporaire

Catégorie $\mathrm{G}$

Catégorie $\mathrm{H}$ Une erreur est survenue causant un dommage permanent au patient Une erreur est survenue entraînant le quasi-décès du patient (ex. : anaphylaxie, arrêt cardiaque...)

\section{Erreur, Mort}

Catégorie I 


\title{
ANNEXE 3 : LISTE DES ORGANISMES AGRÉÉS AU QUEBEC PAR LE CCASS (Conseil Canadien d'Agrément des services de santé)
}

\author{
Source : http://www.cchsa.ca
}

\section{Légende}

\author{
A Établissement de soins de courte durée \\ AMB Service ambulanciers \\ C Centre anticancéreux \\ $\mathrm{CHC} \quad$ Centre de santé communautaire \\ CHS Centre de services communautaires \\ CHS/SS Services de santé communautaire et services sociaux \\ $\mathrm{CMH} \quad$ Services communautaires de santé mentale \\ FNIAS Services de toxicomanie des Premières nations et des Inuits \\ FNICHS Services de santé communautaire des Premières nations et des Inuits \\ $\mathrm{HC} \quad$ Organisme de soins et de services à domicile \\ $\mathrm{L} \quad$ Établissement d'hébergement et de soins de longue durée \\ M Organisme de santé mentale \\ NS Poste de soins infirmiers \\ $\mathrm{PH} \quad$ Services de santé publique \\ $\mathrm{R} \quad$ Centre de réadaptation \\ $\mathrm{R}-\mathrm{ABI} \quad$ Lésions cérébrales acquises \\ * Indique un complexe \\ ${ }^{* *}$ Agrément national
}




\author{
Alma \\ Centre Le Jeannois *A \\ P Pavillon de l'Hôtel-Dieu d'Alma (A) \\ P Pavillon de Métabetchouan (A) \\ P Pavillon du Bel-Âge (L) \\ P Pavillon Le normandie (L) \\ $P$ Pavillon Le pionnier $(\mathrm{L})$ \\ Amos \\ Centre hospitalier Hôtel-Dieu d'Amos A \\ Beaupré \\ Hôpital Sainte-Anne-de-Beaupré A \\ Beauport \\ Centre hospitalier Robert-Giffard M \\ Buckingham \\ CH-CHSLD de Papineau A \\ Chandler \\ Centre hospitalier de Chandler A \\ Chicoutimi \\ Complexe hospitalier de la Sagamie *A \\ P Pavillon Roland Saucier (M) \\ P Pavillon St-Vallier (A) \\ Cote St-Luc \\ Griffith McConnell Residence L \\ Cowansville \\ Hôpital Brome-Missisquoi-Perkins A \\ Drummondville \\ Hôpital Sainte-Croix A \\ Gaspé \\ Centre hospitalier de Gaspé *A \\ P Pavillon Hôtel-Dieu (A) \\ P Pavillon Mgr Ross (A) \\ Gatineau \\ Centre hospitalier des Vallées de l'Outaouais * $A$ \\ $P$ Hôpital de Gatineau (A) \\ P Hôpital de Hull (A) \\ Granby \\ Centre hospitalier de Granby *A \\ $P$ Centre Marie-Berthe Couture $(L)$ \\ Greenfield Park \\ Hôpital Charles LeMoyne A \\ Hull \\ Centre hospitalier Pierre-Janet M \\ Île Bizar \\ Centre d'accueil Denis-Benjamin Viger (CHSLD) L \\ Jonquière \\ Carrefour de la route et des services sociaux de \\ Jonquière * $A$ \\ P Pavillon Arvida $(\mathrm{L})$ \\ $P$ Résidence des Années d'Or (L) \\ $P$ Résidence Georges-Hébert $(L)$ \\ $P$ Résidence Sainte-Marie $(L)$ \\ Lachine \\ Centre hospitalier de Lachine A \\ La Malbaie
}

Centre hospitalier St-Joseph de la Malbaie *A

$P$ Accueil Bellerive $(L)$

$P$ Foyer Notre-Dame du Sacré Coeur de St-

Siméon(L)

$P$ Foyer de Clermont $(L)$

La Salle

Centre hospitalier de La Salle A

Laval

Cité de la santé de Laval $A$

Jewish Rehabilitation Hospital/Hôpital juif de réadaptation $\mathrm{R}$

Lévis

CHA Hôtel-Dieu de Lévis A

Loretteville

Centre de santé de la Haute-Saint-Charles A

Maniwaki

Wanaki Centre FN\&I

Mansfield

Le CLSC, le CHSLD et le Centre hospitalier du Pontiac ${ }^{*} \mathrm{~A}$

P Centre hospitalier du Pontiac (A)

P CHSLD - Fort Coulonge (A)

P CHSLD - Shawville (A)

P CLSC (A)

Maria

Centre hospitalier Baie-des-Chaleurs A

Matane

Les Centre hospitalier et centre d'hébergement et

de soins de longue durée de Matane *A

$P$ Centre hospitalier de Matane $(A)$

$P$ Foyer d'accueil de Matane (CHSLD) (L)

Matane (cont'd/suite)

Pavillon de la Mer inc. (A)

$P$ Ressources intermédiaires $(A)$

Montmagny

Hotel-Dieu de Montmagny A

Montréal

Centre gériatrique Maimonides Hospital Geriatric Centre

$\mathrm{L}$

Centre hospitalier de l'Université de Montréal *A

P Hôpital Hôtel-Dieu du CHUM (A)

P Hôpital Notre-Dame du CHUM (A)

$P$ Hôpital Saint-Luc du CHUM (A)

Centre hospitalier Fleury A

Centre hospitalier Jacques-Viger * $\mathrm{L}$

P Pavillon Ernest-Routhier (L)

Centre hospitalier Mont-Sinaï/Mount Sinai Hospital

CenterA

Centre hospitalier Richardson/Richardson Hospital

Centre L

Centre universitaire de santé McGill/McGill University

Health Centre *A

$P$ L'Hôpital de Montréal pour enfants/ The Montreal

Children's Hospital (A) 
P The Montreal General Hospital/Centre hospitalier de Montréal $(A)$

$P$ Montreal General Hospital/Hôpital général de Montréal( $A)$

P Montreal Neurological Institute/Institut neurologique de Montréal $(A)$

P Royal Victorial Hospital/Hôpital Royal Victoria (A)

CHSLD Les Havres *L

$P$ Havre les Quatres-Saisons $(L)$

$P$ Havre les Quatres-Temps $(L)$

CHSLD Saint-Michel L

Foyer Rousselot $\mathrm{L}$

Hôpital Catherine Booth A

Hôpital du Sacré-Coeur de Montréal *A

P Pavillon Albert Prévost $(L)$

Hôpital Jean-Talon A

Hôpital Louis-H. Lafontaine M

Hôpital Maisonneuve-Rosemont A

Hôpital Notre-Dame de la Merci L

Hôpital Rivière-des-Prairies M

Hôpital Sainte-Justine - Le centre hospitalier

universitaire

mère-enfant ${ }^{*} A$

P Hôpital Marie-Enfant (R)

Hôpital Santa Cabrini Ospedale *A

$P$ Centre d'accueil Dante $(L)$

Hôpital Villa Medica Inc. R

Institut de cardiologie de Montréal *A

$P$ Centre EPIC (R)

Institut de réadaptation de Montréal $R$

Institut Philippe Pinel de Montréal * $M$

$P$ Centre de psychiatrie légale de Montréal (M)

Institut universitaire de gériatrie de Montréal ${ }^{*} \mathrm{~L}$

P Pavillon Alfred-DesRochers ( $L$ )

$P$ Pavillon Cote-des-neiges $(L)$

Medigas Division of Praxair Canada Inc. - Québec * $\mathrm{HC}$

$\mathrm{g}$ All Sites/Tous les bureaux et succursales

Résidences Mance-Décary (C.H.S.L.D.)/Pavillon Saint-

Henri L

St. Mary's Hospital Center/Centre hospitalier Ste-MaryA

Shriners Hospital for Children (Québec) Inc./L'Hôpital

Shriners pour l'enfant infirme A

Sir Mortimer B. Davis - Jewish General Hospital/

Hôpital général juif Sir Mortimer B. Davis A

VitalAire Healthcare ${ }^{*} \mathrm{HC}$

g All Sites/Tous les bureaux et succursales

Ormstown

Hôpital Barrie Memorial A

Pointe-Claire

The Lakeshore General Hospital/Hôpital général

de Lakeshore A

Québec

Centre hospitalier affilié universitaire de Québec *A

$P$ Centre d'hébergement Alphonse-Bonenfant $(L)$
$P$ Centre d'hébergement du Fargy $(L)$

$P$ Centre d'hébergement Maizerets $(L)$

$P$ Centre d'hébergement St-Augustin (L)

$P$ Centre d'hébergement Yvonne-Sylvain (L)

$P$ Hôpital de l'Enfant-Jésus (A)

P Hôpital du Saint-Sacrement (A)

Centre hospitalier universitaire de Québec *A

P CHUL (A)

P Hôpital Hôtel-Dieu de Québec (A)

P Hôpital Saint-François d'Assise (A)

CLSC-CHSLD Haute-Ville-Des-Rivières *L

$P$ Résidence Christ-Roi (L)

$P$ Résidence Le Faubourg (L)

$P$ Résidence Louis-Hébert ( $L$ )

$P$ Résidence Sacré-Coeur (L)

$P$ Résidnece St-Antoine (L)

P Site 383, chemin Sainte-Foy (HC)

P Site 530, de l'Atrium (HC)

Hôpital Jeffery Hale L

Repentigny

Centre hospitalier Le Gardeur A

Rimouski

Centre hospitalier régional de Rimouski A

Rivière-du-Loup

Le Centre Hospitalier de Rivière-du-Loup *A

$P$ Centre hospitalier régional du Grand-Portage (A)

$P$ Centre d'hébergement Saint-Joseph ( $L$ )

$P$ Foyer de Saint-Antonin (L)

Rouyn-Noranda

Centre hospitalier Rouyn-Noranda A

Saint-Hyacinthe

Réseau Santé Richelieu-Yamaska *A

$P$ Pavillon Honoré-Mercier (A)

P Pavillon Hôtel-Dieu (A)

P Pavillon St-Charles $(L)$

Saint-Jérôme

Hôtel-Dieu de Saint-Jérôme A

Shawinigan

Centre hospitalier du Centre-de-la-Mauricie *M

$P$ Centre d'intérêt communautaire/Shawinigan (M)

$P$ Centre d'intérêt communautaire/Trois-Rivières (M)

$\mathrm{P}$ Centre de travail S. Barolet (M)

P Pavillon Bergeron (M)

P Pavillon Gérard \& Parise Dupuis (M)

$P$ Pavillon Jacques Lefrançois (M)

P Pavillon La Résidence Les Chutes (M)

P Pavillon Mona Lisa (M)

P Pavillon Plessis (M)

P Pavillon St-Boniface (M)

P Pavillon St-Paulin 1994 Enr. (M)

$P$ Site Sainte-Thérèse $(M)$

$P$ Site Shawinigan-Sud (M) 
Centre hospitalier de la région de l'Amiante $\mathrm{A}$

\author{
Sherbrooke \\ Centre hospitalier universitaire de Sherbrooke *A \\ $P$ Centre hospitalier universitaire Hôpital Fleurimont( $A$ ) \\ Institut universitaire de gériatrie de Sherbrooke *A \\ P Pavillon Argyll (L) \\ P Pavillon D'Youville (A) \\ St-Charles-Borromée \\ Centre hospitalier régional de Lanaudière ${ }^{*} \mathrm{~A}$ \\ $P$ Centre d'accueil St-Eusèbe $(L)$ \\ $P$ Centre d'accueil St-Thomas (L) \\ $P$ Pédopsychiatrie (M) \\ Ste-Anne-de-Bellevue \\ Hôpital Sainte-Anne L \\ Ste-Anne-des-Plaines \\ Centre régional de santé mentale $\mathrm{M}$ \\ Ste-Foy \\ Hôpital Laval A \\ St-Jean-sur-Richelieu \\ Hôpital du Haut-Richelieu *A \\ $P$ Centre Georges Phaneuf (A) \\ P Centre Gertrude Lafrance (A) \\ Témiskaming \\ Centre de santé de Témiskaming A< \\ Thetford-Mines
}

\section{Trois-Rivières}

Centre hospitalier régional de Trois-Rivières * $A$

$P$ Carrefour Niverville $(L)$

$P$ Pavillon Arc-en-Ciel (L)

P Pavillon Saint-Joseph (A)

P Pavillon Sainte-Marie (A)

Val-d'Or

Centre hospitalier de Val-d'Or A

Valleyfield

Centre hospitalier régional du Suroît A

Varennes

CHSLD du Litoral *L

$P$ Centre Contrecoeur (L)

$P$ Centre Jeanne-Crevier ( $L$ )

P CHSLD Foyer Lajemmerais (L)

$P$ Pavillon St-Patrick $(L)$

$P$ Résidence de Bouchervile (L)

Verdun

Centre hospitalier de Verdun A

Douglas Hospital/Hôpital Douglas M

Victoriaville

Hôtel-Dieu d'Arthabaska A

Ville de Saint-Georges

Centre hospitalier Beauce-Etchemin A 


\section{BIBLIOGRAPHIE}

Amalberti R., 1996, La conduite des systèmes à risques. Paris : PUF.

Agence Nationale d'Accrédiation et d'Evaluation en Santé (ANAES), 2003, Principes méthodologiques pour la gestion des risques en établissement de santé, rapport, Janvier.

Australian Council for Safety and Quality in Health Care. 2001. Safety in Practice: Making Health Care Safer. Second Report to the Australian Health Ministers' Conference.

Australian Council for Safety and Quality in Health Care. 2001. National Action Plan 2001.Canberra: Australian Council for Safety and Quality in Health Care.

Baker, R., and Norton, P. 2001. Making Patients Safer! Reducing Error in Canadian healthcare. HealthC

Battles, J. B. (2001). « Disaster prevention: Lessons learned from the Titanic ». Baylor University Medical Center Proceedings 14: 150-153.

Baumont, G. N. Matahri, J.L Roy, 1999, "RECUPERARE, a model of event including human reliability in nuclear Plants", Proceedings of PSAM 5th Conférence, vol 1/4, Universal academy press , INC Tokyo Japan.

Baumont, G., N. Matahri, S. Bardou, 2000, «Modèle d'analyse des incidents RECUPERARE, intégrant les facteurs techniques, humains et organisationnels », Revue Préventique, Octobre.

Brennan, T.A., L.L. Leape, et al. 1991. Incidence of Adverse Events and Negligence in Hospitalized Patients. N Engl J Med 324(6): 370 - 377.

Carroll, R, (Editor), 2001, Risk Management Handbook for Health Care Organizations, 3rd Edition, Jossey-Bass.

Chopard P, Mabboux M., 2001, Analyse des incidents en médecine: nouvelles perspectives et analyse de cas. Med Hyg ;59: 1077-9.

Chopard, P, Wasserfallen, J.B, 2002, Gestion des incidents dans les organisations de soins, Rapport FoQual, Mai.

Cooper, J. B., R. S. Newbower et coll., 1978, « Preventable anesthesia mishaps: a study of human factors ». Anesthesiology 49(6): 399-406.

De Marcellis-Warin N, S Gauthier-Gaillard, 2001, Gestion et prévention des risques iatrogènes : une étude comparative en milieu hospitalier entre la France et l'Etranger, Rapport remis au Ministère de l'Emploi et de la Solidarité - Secrétariat d'Etat à la Santé - Direction de l'Hospitalisation et de l'organisation des soins (France). 
De Marcellis-Warin N., 2003, "What the Management of Industrial Risk has to say about Health Care Risk Management », Document de travail CIRANO, à paraître.

De Marcellis-Warin N., G. Dufour, 2003, « Déclaration des incidents et des accidents dans les centres hospitaliers - Analyse critique du rapport d'incident/accident AH-223 et de la structure de gestion de ces rapports », rapport de recherche CIRANO remis au Ministère de la Santé et des Services Sociaux du Québec, avril.

Donchin, Y., D. Gopher et coll., 1995, « A look into the nature and causes of human errors in the intensive care unit ». Critical Care Medicine 23(2): 294-300.

Gaba, DM, 2000, “Anaesthesiology as a model for patient safety in health care”, British Medical Journal, 320:785-788 (18 March).

Gourdeau, M., «Les infections nosocomiales, un risque évitable », L'événement, publication de l'AHQ, vol 14, n.1, avril 2003.

Helmreich, RL, 2000, “On error management: lessons from aviation”, British Medical Journal, 320:781-785 (18 March).

Helmreich, RL, DM Musson, 2000, "The University of Texas Threat and Error Management Model : Components and Examples", British Medical Journal.

Institute of Medicine. 2001. Crossing the Quality Chasm: A New Health System for the $21^{\text {st }}$ Century. Washington: National Academy Press.

Institute of Medicine. 1999. To Err is Human: Building a Safer Health System. Washington: National Academy Press.

Kohn, L. T., J. M. Corrigan, et al., eds. 1999. To Err is Human: Building a Safer Health System. Washington: National Academy Press.

Joint Commission on Accreditation on Health Organisations, 2001, Preventing Adverse Events in Behavioral Health Care : a System Approach to Sentinel Events, Report.

Leape, L.L., Brennan, T.A., et al. 1991. The Nature of Adverse Events in Hospitalized Patients . N Engl J Med 324 (6), 377-384.

Leape, LL, 1994, "Error in Medicine”, JAMA 272, pp1851-1857.

Leape, LL, DW Bates, DJ Cullen, 1995, "Systems Analysis of Adverse Drug Events", JAMA 274, pp 35-43.

Lefebvre, P., M. Beauchemin Perreault, 2001, «Un bref aperçu du rapport du Comité ministériel : Les accidents évitables dans la prestation des soins de santé », Pharmactuel Vol. 34 No 6 Novembre-Décembre. 
Mayor, S. 2001. NHS introduced new patient safety agency. British Medical Journal 322(28): 1013.

McKerrow, R., Rappaport, P., Bussières, J.F., Hall, K., Long, S and Salsman, B. Hospital Pharmacy in Canada Annual Report 1999/2000. Toronto, Canada: Eli Lilly. 2000.

Ministère de la Santé et des Services Sociaux du Québec, 2001, Les accidents évitables dans la prestation des soins : la gestion des risques, une priorité pour le réseau, Rapport du comité Francoeur, Février.

National Health Service. 2000. An Organization with a Memory: Report of an Expert Group on Learning from Adverse Events in the NHS. London: Department of Health.

National Heath Service. 2001. Building A Safer NHS for Patients: Implementing an Organization with a Memory. London: Department of Health.

National Health Service. 2001. Doing Less Harm: Key Requirements for Health Care Providers. London: Department of Health.

Murray Gell-Mann, 1995, "What is complexity ?" , Complexity, Vol. 1, no. 1, 1995. John Wiley and Sons, Inc.

New Zealand Ministry of Health, 2001, Toward Clinical Excellence : Learning from experience, Report to the Director General of Health.

Paté-Cornell, ME, LM Lakats, DM Murphy and DM Gaba, 1997, "Anesthesia Patient Risk : A quantitative Approach to organisational Factors and Risk Management Options, Risk Analysis", Vol.17, n.4, pp511-523.

Perrow, Charles, 1984, Normal accidents, New York:Basic Books.

Reason, J, 1990, Human Error. Cambridge, Mass: Cambridge University Press.

Reason J, 1995, Understanding adverse events: human factors. In: Vincent CA, ed. Clinical risk management. London: BMJ Book; 1995. p. 31-54.

Reason, J, 1997, Managing the Risks of Organizational Accidents, Ashgate editor.

Spath, PL, (Editor), 2000, Error Reduction in Health Care: A Systems Approach to Improving Patient Safety, San Francisco, Calif, Jossey-Bass Publishers, Chicago, Ill, Health Forum, 2000.

Spathe, P. 1998. Medical errors: Root Cause Analysis. Operating Room Manager, 14 (9), 38- 41.

Verot, Y., 1999, Maîtrise du risque : le retour d'expérience, Ecole d'été "Gestion Scientifique du risque " 6/10 septembre, ALBI - France.

Villemeur A. 1988, Sûreté de fonctionnement des systèmes industriels : fiabilité, facteurs humains, information. Paris: Eyrolles. 
Vincent C., 1995, ed. Clinical risk management. London: BMJ Book.

Vincent C., Adams S, Stanhope N., 1998, A framework for the analysis of risk and safety in Medicine, British Medical Journal 316: 1154-1157.

Vincent C., 2001, Clinical risk management. Enhancing patient safety. London: BMJ Book.

Vincent C, Taylor-Adams S, Stanhope N., 1998, Framework for analysing risk and safety in clinical medicine. BMJ ; 316(7138):1154-7.

Vincent C, Taylor-Adams S, Chapman EJ, Hewett D, Prior S, Strange P et al. , 2000, How to investigate and analyse clinical incidents: clinical risk unit and association of litigation and risk management protocol, BMJ;320(7237):777-81.

Vincent C, Stanhope N, Crowley-Murphy M., 1999, Reasons for not reporting adverse incidents: an empirical study. J Eval Clin Pract ;5(1):13-21.

Vincent, C., G. Neale, et al. 2001. Adverse Events in British Hospitals: Preliminary

Retrospective Record Review. British Medical Journal 322(7285): 517-519.

Wanzel, K.R., Jamieson, C.G., and Bohnen, J.M. 2000. Complications on a General Surgery Service: Incidence and Reporting. Can J Surg 43 (2):113-7.

Wilson, RM, and alii, 1995, "The quality in Australian health care study", The Medical Journal of Australia, vol. 163, November.

Wilson, R., Harrison, B., Gibberd, R., and Hamilton, J. 1999. An Analysis of the Causes of Adverse Events from the Quality in Australian Health Care Study. Med J Aust 170: 411-415.

Wilson, R.M., Runciman, W.G., and Gibberd, R.W., et al. 1995. The Quality in Australian Health Care Study. Med J Aust 163:458-471.

Wiener, E.L (eds.), 1988, Human Factors in Aviation, Orlando, FL:Academics Press, Inc.

World Health Organization. 2002. Quality of Care: Patient Safety. Report by the Secretariat.

Zipperer, L., and Cushman, S., eds. 2001. Lessons in Patient Safety. Chicago: National Patient Safety Foundation. 
